OAK RIDGE
NATIONAL LABORATORY

MANAGED BY UT-BATTELLE

FOR THE DEPARTMENT OF ENERGY
ORNL/TM-2006/507

\section{Data Compilation for AGR-1 Baseline Compact Lot LEU01-46T-Z}

\author{
Revision 0
}

\section{John D. Hunn, Fred C. Montgomery and Peter J. Pappano}

This report was prepared as an account of work sponsored by an agency of the United States Government. Neither the United States Government nor any agency thereof, or any of their employees, makes any warranty, expressed or implied, or assumes any legal liability or responsibility for any third party's use, or the results of such use, of any information, apparatus, product or process disclosed in this report, or represents that its use by such third party would not infringe privately owned rights. 


\section{DOCUMENT AVAILABILITY}

Reports produced after January 1, 1996, are generally available free via the U.S. Department of Energy (DOE) Information Bridge.

Web site http://www.osti.gov/bridge

Reports produced before January 1, 1996, may be purchased by members of the public from the following source.

National Technical Information Service

5285 Port Royal Road

Springfield, VA 22161

Telephone 703-605-6000 (1-800-553-6847)

TDD 703-487-4639

Fax 703-605-6900

E-mail info@ntis.fedworld.gov

Web site http://www.ntis.gov/support/ordernowabout.htm

Reports are available to DOE employees, DOE contractors, Energy Technology Data Exchange (ETDE) representatives, and International Nuclear Information System (INIS)

representatives from the following source.

Office of Scientific and Technical Information

P.O. Box 62

Oak Ridge, TN 37831

Telephone 865-576-8401

Fax 865-576-5728

E-mail reports@adonis.osti.gov

Web site http://www.osti.gov/contact.html

This report was prepared as an account of work sponsored by an agency of the United States Government. Neither the United States Government nor any agency thereof, nor any of their employees, makes any warranty, express or implied, or assumes any legal liability or responsibility for the accuracy, completeness, or usefulness of any information, apparatus, product, or process disclosed, or represents that its use would not infringe privately owned rights. Reference herein to any specific commercial product, process, or service by trade name, trademark, manufacturer, or otherwise, does not necessarily constitute or imply its endorsement, recommendation, or favoring by the United States Government or any agency thereof. The views and opinions of authors expressed herein do not necessarily state or reflect those of the United States Government or any agency thereof. 


\title{
Data Compilation for AGR-1 Baseline Compact Lot LEU01-46T-Z
}

\author{
J. D. Hunn, F. C. Montgomery and P. J. Pappano \\ Oak Ridge National Laboratory
}

This document is a compilation of characterization data for the AGR-1 baseline compact lot LEU01-46T-Z. The compacts were produced by ORNL for the Advanced Gas Reactor Fuel Development and Qualification (AGR) program for the first AGR irradiation test train (AGR-1). This compact lot was fabricated using particle composite LEU01-46T, which was a composite of four batches of TRISO-coated $350 \mu \mathrm{m}$ diameter 19.7\% low enrichment uranium oxide/uranium carbide kernels (LEUCO). The AGR-1 TRISO-coated particles consist of a spherical kernel coated with an $\sim 50 \%$ dense carbon buffer layer (100 $\mu \mathrm{m}$ nominal thickness), followed by a dense inner pyrocarbon layer ( $40 \mu \mathrm{m}$ nominal thickness), followed by a SiC layer ( $35 \mu \mathrm{m}$ nominal thickness), followed by another dense outer pyrocarbon layer (40 $\mu \mathrm{m}$ nominal thickness). The kernels were obtained from BWXT and identified as composite G73D-20-69302. The BWXT kernel lot G73D-20-69302 was riffled into sublots for characterization and coating by ORNL and identified as LEU01-\#\# (where \#\# is a series of integers beginning with 01). A data compilation for the AGR-1 baseline coated particle composite LEU01-46T can be found in ORNL/TM2006/019.

The AGR-1 Fuel Product Specification and Characterization Guidance (INL EDF-4380) provides the requirements necessary for acceptance of the fuel manufactured for the AGR-1 irradiation test. Section 6.2 of EDF-4380 provides the property requirements for the heat treated compacts. The Statistical Sampling Plan for AGR Fuel Materials (INL EDF-4542) provides additional guidance regarding statistical methods for product acceptance and recommended sample sizes. The procedures for characterizing and qualifying the compacts are outlined in ORNL product inspection plan AGR-CHAR-PIP-05. The inspection report forms generated by this product inspection plan document the product acceptance for the property requirements listed in section 6.2 of EDF-4380. 


\section{Table of Contents}

1 Summary of acceptance test results for LEU01-46T-Z

2 Compacting process conditions

3 Characterization of compacts 18 


\section{Summary of acceptance test results for LEU01-46T-Z}

This section contains inspection report forms (IRFs) associated with the compact lot LEU0146T-Z. These inspection report forms also appear in a later section of this compilation, accompanied by the associated data report forms (DRFs) showing the results of each individual measurement. These inspection report forms summarize the acceptance testing performed according to the product inspection plan AGR-CHAR-PIP-05. The information in these forms covers all the property specifications listed in section 6.2 of the AGR-1 Fuel Product Specification and Characterization Guidance document INL EDF-4380, Rev. 8. The compact lot, LEU01-46T-Z, was found to meet all the requirements in section 6.2 of EDF-4380, Rev. 8 with the exception of 19 compacts which were available for irradiation that did not meet the specified minimum length. These 19 non-conforming compacts have been dispositioned for use as is by NCR-X-AGR-06-03.

Table 1-1 is provided for quick reference. It gives the mean values of key variable properties of the compact lot, LEU01-46T-Z. For standard deviations of the distribution of the measured values see the appropriate IRF or DRF. For discussions on the uncertainty in these values, see the associated data acquisition methods and data report forms.

Table 1-1: Quick reference table for key variable properties of LEU01-46T-Z.

\begin{tabular}{|l|c|}
\hline \multicolumn{1}{|c|}{ Property } & Mean \\
\hline \hline Mean uranium loading $(\mathrm{g} \mathrm{U} /$ compact$)$ & 0.917 \\
\hline Compact diameter $(\mathrm{mm})$ & 12.37 \\
\hline Compact length $(\mathrm{mm})$ & 25.05 \\
\hline Compact mass $(\mathrm{g})$ & 5.482 \\
\hline Impurity content & Table 1-2 \\
\hline
\end{tabular}

The mean impurity levels for the fuel compacts reported on IRF-05A and IRF-05B are probably higher than the actual values for two reasons. First, the as-reported mean impurity levels do not reflect the fact that some of the measurements were at or below the measurement threshold and could not be differentiated from zero. Second, the as-reported mean impurity levels do not account for impurities introduced during the analysis.

Each time a leach was performed, a blank run was also performed where all the relevant wet chemistry steps in the leach-burn-leach procedure in AGR-CHAR-DAM-26R0 were performed without a compact present in order to obtain background values for the analyzed impurities. Table 1-2 shows the total as-reported mean and standard deviation for each measured impurity as well as the total mean values adjusted by subtracting the background values obtained from the associated blank runs. In cases where the value for the blank run was reported as being below a certain measurement threshold value, a minimum value for that leach was calculated by subtracting the threshold value and a maximum value was calculated by subtracting zero. The adjusted mean therefore accounts for all the measurable impurities in the blanks and spans a range that reflects the uncertainty due to the measurement thresholds. 
Table 1-2: Mean impurity levels for fuel compacts from LEU-46T-Z compact lot measured by deconsolidation leach-burn-leach technique.

\begin{tabular}{|c|c|c|c|}
\hline \multirow{2}{*}{ Measured Impurity } & \multirow{2}{*}{$\begin{array}{c}\text { As-reported } \\
\text { Mean }\end{array}$} & \multicolumn{2}{|c|}{ Adjusted Mean } \\
\hline & & Mininum & Maximum \\
\hline Fe outside SiC ( $\mu \mathrm{g} /$ compact): & $2.97 \pm 0.56$ & $1.46 \pm 0.72$ & $1.57 \pm 0.59$ \\
\hline Cr outside SiC ( $\mu \mathrm{g} /$ compact): & $1.58 \pm 0.02$ & $0.00 \pm 0.00$ & $1.58 \pm 0.02$ \\
\hline Mn outside SiC $(\mu \mathrm{g} /$ compact $):$ & $0.42 \pm 0.08$ & $0.02 \pm 0.04$ & $0.12 \pm 0.03$ \\
\hline Co outside SiC ( $\mu \mathrm{g} /$ compact): & $0.63 \pm 0.01$ & $0.00 \pm 0.00$ & $0.63 \pm 0.01$ \\
\hline Ni outside SiC ( $\mu \mathrm{g} /$ compact): & $1.20 \pm 0.11$ & $0.08 \pm 0.10$ & $1.20 \pm 0.11$ \\
\hline $\mathbf{C r}+\mathbf{M n}+\mathbf{C o}+\mathbf{N i}$ outside SiC ( $\mu \mathrm{g} /$ compact $):$ & $3.82 \pm 0.19$ & $0.10 \pm 0.14$ & $3.53 \pm 0.17$ \\
\hline Ca outside SiC ( $\mu \mathrm{g} /$ compact): & $14.77 \pm 1.91$ & $7.45 \pm 1.90$ & $7.95 \pm 1.92$ \\
\hline Al outside SiC ( $\mu \mathrm{g} /$ compact): & $10.28 \pm 1.51$ & $3.84 \pm 1.35$ & $8.54 \pm 1.16$ \\
\hline Ti outside SiC ( $\mu \mathrm{g} / \mathrm{compact}):$ & $9.00 \pm 1.17$ & $6.39 \pm 1.00$ & $6.96 \pm 1.00$ \\
\hline V outside SiC ( $\mu \mathrm{g} /$ compact): & $18.20 \pm 1.42$ & $17.28 \pm 1.45$ & $18.20 \pm 1.42$ \\
\hline $\mathbf{T i}+\mathbf{V}$ outside SiC $(\mu \mathrm{g} /$ compact $):$ & $27.20 \pm 2.33$ & $23.67 \pm 2.13$ & $25.15 \pm 2.11$ \\
\hline
\end{tabular}

Table 1-3 is also provided for quick reference. It gives the upper limit of the $95 \%$ confidence interval of the defect fraction for key attribute properties of the compact lot LEU01-46T-Z. In other words, these values are the lowest tolerance limits for which the compact lot would be deemed acceptable at 95\% confidence based on the particular sample that was measured. For the actual number of trials and number of failures observed, see the inspection report form for the compact lot.

Table 1-3: Quick reference table for key attribute properties of LEU01-46T-Z.

\begin{tabular}{|l|c|}
\hline \multicolumn{1}{|c|}{ Property } & Defect Fraction \\
\hline Uranium contamination fraction & $\leq 3.1 \cdot 10^{-5}$ \\
\hline Defective SiC coating fraction & $\leq 1.3 \cdot 10^{-4}$ \\
\hline Defective IPyC coating fraction & $\leq 6.1 \cdot 10^{-5}$ \\
\hline Defective OPyC coating fraction & $\leq 7.3 \cdot 10^{-4}$ \\
\hline
\end{tabular}

Also worthy of note is the observation of particles with SiC layers less than $20 \mu \mathrm{m}$ thick. These were observed and noted for information only during x-ray analysis for uranium dispersion after compacting due to defective IPyC. In terms of the upper limit of the $95 \%$ confidence interval of the anomaly fraction, as reported in Table 1-3, the fraction of particles in the compact lot with $\mathrm{SiC}<20 \mu \mathrm{m}$ thick is $\leq 4.7 \cdot 10^{-4}$. This anomaly is probably caused by particles being temporarily trapped in carbon soot that has built up on the walls of the coating chamber above the fluidized particle bed. This is the same mechanism thought to cause soot inclusions (goldspots) within the $\mathrm{SiC}$ layer. 
DRF-24 indicates that 30 compacts failed to meet the length specification, being shorter than the lower acceptance limit of $25.02 \mathrm{~mm}$. Of the 30 compacts that were shorter than $25.02 \mathrm{~mm}, 11$ were selected for destructive characterization according to AGR-CHAR-PIP-05R0. The remaining 19 compacts that were shorter than $25.02 \mathrm{~mm}$ were available for irradiation and dispositioned for use as is by NCR-X-AGR-06-03. Table 1-4 lists the compacts that are available for irradiation sorted in order of increasing length.

Table 1-4: Compacts from LEU-46T-Z compact lot available for irradiation sorted by length

\begin{tabular}{|c|c|c|c|c|c|c|c|c|c|c|}
\hline \multirow{2}{*}{$\begin{array}{l}\text { Compact } \\
\text { ID Number }\end{array}$} & \multirow{2}{*}{$\begin{array}{c}\text { Length } \\
(\mathrm{mm})\end{array}$} & \multicolumn{6}{|c|}{ Diameter ( $\mathrm{mm})$} & \multirow{2}{*}{$\begin{array}{c}\text { Pass Thru? } \\
\text { ( } \mathrm{Y} \text { or } \mathrm{N})\end{array}$} & \multirow{2}{*}{$\begin{array}{c}\text { Mass } \\
(\mathrm{g})\end{array}$} & \multirow{2}{*}{$\begin{array}{c}\text { Accept? } \\
\text { (pass or fail) }\end{array}$} \\
\hline & & Top 1 & Top 2 & Middle 1 & Middle 2 & Bottom 1 & Bottom 2 & & & \\
\hline 03 & 24.857 & 12.40 & 12.41 & 12.41 & 12.41 & 12.41 & 12.41 & $\overline{Y Y}$ & 5.4757 & fail \\
\hline 68 & 24.928 & 12.37 & 12.36 & 12.37 & 12.37 & 12.36 & 12.36 & $\bar{Y}$ & 5.4756 & fail \\
\hline 37 & 24.938 & 12.38 & 12.37 & 12.37 & 12.37 & 12.36 & 12.36 & $\bar{Y}$ & 5.4757 & fail \\
\hline 38 & 24.941 & 12.37 & 12.37 & 12.38 & 12.38 & 12.37 & 12.38 & $\mathrm{Y}$ & 5.4797 & fail \\
\hline 76 & 24.942 & 12.37 & 12.37 & 12.37 & 12.37 & 12.36 & 12.37 & $\mathrm{Y}$ & 5.4706 & fail \\
\hline 78 & 24.960 & 12.36 & 12.36 & 12.36 & 12.37 & 12.36 & 12.36 & $\mathrm{Y}$ & 5.4374 & fail \\
\hline 18 & 24.960 & 12.37 & 12.37 & 12.38 & 12.37 & 12.36 & 12.36 & $\mathrm{Y}$ & 5.4730 & fail \\
\hline 12 & 24.985 & 12.37 & 12.37 & 12.37 & 12.37 & 12.36 & 12.36 & $\mathrm{Y}$ & 5.4890 & fail \\
\hline 79 & 24.985 & 12.37 & 12.37 & 12.37 & 12.37 & 12.36 & 12.36 & $\mathrm{Y}$ & 5.4721 & fail \\
\hline 27 & 24.992 & 12.38 & 12.38 & 12.38 & 12.38 & 12.36 & 12.36 & $\mathrm{Y}$ & 5.4936 & fail \\
\hline 23 & 24.994 & 12.37 & 12.37 & 12.37 & 12.37 & 12.36 & 12.36 & $\mathrm{Y}$ & 5.4814 & fail \\
\hline 74 & 24.996 & 12.37 & 12.37 & 12.38 & 12.38 & 12.36 & 12.36 & $\mathrm{Y}$ & 5.4793 & fail \\
\hline 77 & 24.997 & 12.36 & 12.37 & 12.37 & 12.37 & 12.36 & 12.36 & $\mathrm{Y}$ & 5.4648 & fail \\
\hline 14 & 24.997 & 12.37 & 12.37 & 12.37 & 12.36 & 12.36 & 12.36 & $\mathrm{Y}$ & 5.4797 & fail \\
\hline 67 & 25.001 & 12.36 & 12.36 & 12.36 & 12.36 & 12.35 & 12.36 & $\mathrm{Y}$ & 5.4546 & fail \\
\hline 20 & 25.003 & 12.38 & 12.38 & 12.38 & 12.38 & 12.37 & 12.37 & $\mathrm{Y}$ & 5.4855 & fail \\
\hline 32 & 25.005 & 12.37 & 12.37 & 12.37 & 12.37 & 12.37 & 12.36 & $\mathrm{Y}$ & 5.4732 & fail \\
\hline 07 & 25.006 & 12.37 & 12.37 & 12.37 & 12.37 & 12.36 & 12.36 & $\bar{Y}$ & 5.4676 & fail \\
\hline 09 & 25.013 & 12.37 & 12.37 & 12.37 & 12.37 & 12.35 & 12.36 & $\mathrm{Y}$ & 5.4786 & fail \\
\hline 53 & 25.022 & 12.37 & 12.37 & 12.37 & 12.37 & 12.37 & 12.36 & $\mathrm{Y}$ & 5.4877 & pass \\
\hline 24 & 25.022 & 12.37 & 12.37 & 12.37 & 12.37 & 12.36 & 12.36 & $\mathrm{Y}$ & 5.4810 & pass \\
\hline 44 & 25.027 & 12.37 & 12.37 & 12.36 & 12.37 & 12.37 & 12.36 & $\mathrm{Y}$ & 5.4851 & pass \\
\hline 15 & 25.030 & 12.37 & 12.37 & 12.37 & 12.37 & 12.36 & 12.36 & $\mathrm{Y}$ & 5.4835 & pass \\
\hline 36 & 25.031 & 12.37 & 12.37 & 12.37 & 12.37 & 12.36 & 12.36 & $\mathrm{Y}$ & 5.4655 & pass \\
\hline 65 & 25.032 & 12.37 & 12.37 & 12.37 & 12.37 & 12.36 & 12.36 & $\mathrm{Y}$ & 5.4856 & pass \\
\hline 69 & 25.036 & 12.37 & 12.37 & 12.37 & 12.37 & 12.35 & 12.36 & $\mathrm{Y}$ & 5.4695 & pass \\
\hline 47 & 25.041 & 12.37 & 12.37 & 12.37 & 12.37 & 12.37 & 12.37 & $\mathrm{Y}$ & 5.4802 & pass \\
\hline 22 & 25.053 & 12.38 & 12.37 & 12.37 & 12.38 & 12.36 & 12.37 & $\mathrm{Y}$ & 5.4980 & pass \\
\hline 42 & 25.059 & 12.37 & 12.37 & 12.36 & 12.36 & 12.35 & 12.36 & $\mathrm{Y}$ & 5.4867 & pass \\
\hline 55 & 25.065 & 12.37 & 12.37 & 12.37 & 12.37 & 12.36 & 12.36 & $Y$ & 5.4907 & pass \\
\hline 56 & 25.073 & 12.37 & 12.36 & 12.37 & 12.37 & 12.36 & 12.37 & $\mathrm{Y}$ & 5.4913 & pass \\
\hline 58 & 25.073 & 12.36 & 12.36 & 12.36 & 12.36 & 12.35 & 12.35 & $\mathrm{Y}$ & 5.4760 & pass \\
\hline 46 & 25.074 & 12.36 & 12.36 & 12.36 & 12.36 & 12.35 & 12.35 & $\bar{Y}$ & 5.4631 & pass \\
\hline 17 & 25.074 & 12.37 & 12.37 & 12.37 & 12.37 & 12.36 & 12.36 & $\bar{Y}$ & 5.4861 & pass \\
\hline 49 & 25.090 & 12.37 & 12.37 & 12.37 & 12.37 & 12.37 & 12.36 & $\mathrm{Y}$ & 5.4791 & pass \\
\hline 34 & 25.097 & 12.37 & 12.36 & 12.37 & 12.36 & 12.36 & 12.36 & $\mathrm{Y}$ & 5.4893 & pass \\
\hline 33 & 25.109 & 12.37 & 12.37 & 12.37 & 12.37 & 12.37 & 12.36 & $\mathrm{Y}$ & 5.5023 & pass \\
\hline 29 & 25.114 & 12.38 & 12.38 & 12.38 & 12.38 & 12.37 & 12.36 & $\mathrm{Y}$ & 5.4826 & pass \\
\hline 30 & 25.115 & 12.37 & 12.36 & 12.37 & 12.37 & 12.35 & 12.36 & $Y$ & 5.4756 & pass \\
\hline 43 & 25.133 & 12.37 & 12.36 & 12.36 & 12.37 & 12.36 & 12.36 & $\mathrm{Y}$ & 5.4941 & pass \\
\hline 62 & 25.135 & 12.36 & 12.36 & 12.36 & 12.36 & 12.35 & 12.35 & $\mathrm{Y}$ & 5.4719 & pass \\
\hline 50 & 25.140 & 12.37 & 12.37 & 12.37 & 12.37 & 12.36 & 12.35 & $\mathrm{Y}$ & 5.4843 & pass \\
\hline 57 & 25.142 & 12.36 & 12.36 & 12.37 & 12.37 & 12.35 & 12.35 & $\bar{Y}$ & 5.4829 & pass \\
\hline 52 & 25.143 & 12.36 & 12.36 & 12.36 & 12.36 & 12.36 & 12.35 & $\mathrm{Y}$ & 5.4792 & pass \\
\hline 39 & 25.147 & 12.37 & 12.37 & 12.37 & 12.37 & 12.36 & 12.36 & $\mathrm{Y}$ & 5.5035 & pass \\
\hline 60 & 25.250 & 12.36 & 12.36 & 12.36 & 12.36 & 12.36 & 12.35 & $\mathrm{Y}$ & 5.4757 & pass \\
\hline 19 & 25.256 & 12.36 & 12.37 & 12.36 & 12.36 & 12.35 & 12.35 & $\mathrm{Y}$ & 5.4874 & pass \\
\hline 05 & 25.264 & 12.36 & 12.36 & 12.36 & 12.36 & 12.35 & 12.36 & $\mathrm{Y}$ & 5.4779 & pass \\
\hline
\end{tabular}


ORNL/TM-2006/507, Rev. 0

Inspection Report Form IRF-05A: Fuel Compact Lots

Procedure: AGR-CHAR-PIP-05 Rev, 0

Compact lot ID: LEU01-46T-Z

Compact lot description: Compacts of AGR-1 baseline coated particle composite LEU01-46

Compact ID numbers of compacts available for irradiation test (pending acceptance): $: \begin{aligned} & 03,05,07,09,12,14,15,17,18,19,20,22,23,24,27,29,30,32,33,34,36,37,38,39 \\ & 42,43,44,46,47,49,50,52,53,55,56,57,58,60,62,65,67,68,69,74,76,77,78,79\end{aligned}$

\begin{tabular}{|c|c|c|c|c|c|c|c|c|c|}
\hline \multirow[b]{2}{*}{ Property } & \multicolumn{4}{|c|}{ Measured Data } & \multirow{2}{*}{$\begin{array}{c}\text { Specification } \\
\text { INL EDF-4380 } \\
\text { Rev. } 8\end{array}$} & \multirow[b]{2}{*}{ Acceptance Criteria } & \multirow{2}{*}{$\begin{array}{l}\text { Acceptance } \\
\text { Test Value }\end{array}$} & \multirow{2}{*}{$\begin{array}{l}\text { Pass } \\
\text { or } \\
\text { fail }\end{array}$} & \multirow{2}{*}{$\begin{array}{l}\text { Data } \\
\text { Records }\end{array}$} \\
\hline & $\begin{array}{l}\text { Mean } \\
(x)\end{array}$ & $\begin{array}{l}\text { Std. Dev. } \\
\text { (s) }\end{array}$ & $\begin{array}{c}\text { Measurements } \\
(n)\end{array}$ & $\begin{array}{l}k \text { or } t \\
\text { value }\end{array}$ & & & & & \\
\hline Compact diameter $(\mathrm{mm})$ & \multirow{2}{*}{\multicolumn{2}{|c|}{ See DRF-24 }} & & & $12.22-12.46$ & all avallable for irradiation test & & pass & DRF-24 \\
\hline Compact length $(\mathrm{mm})$ & & & & & $25.02-25.40$ & meet specification & & (Note 1) & DRr -24 \\
\hline \multirow{2}{*}{$\begin{array}{l}\text { Uranium loading } \\
\text { (gU/compact) }\end{array}$} & \multirow{2}{*}{0.917} & \multirow{2}{*}{0.005} & \multirow{2}{*}{6} & \multirow{2}{*}{2.015} & \multirow{2}{*}{$0.905 \pm 0.04$} & $A=x-t s / \sqrt{ } n \geq 0.865$ & 0.913 & pass & \multirow{2}{*}{ DRF-25 } \\
\hline & & & & & & $B=x+t s / V n \leq 0.945$ & 0.921 & pass & \\
\hline \multirow{2}{*}{$\begin{array}{l}\text { Iron content outside SiC } \\
\text { ( } \mu g / \text { compact) }\end{array}$} & \multirow{2}{*}{2.97} & \multirow{2}{*}{0.56} & \multirow{2}{*}{4} & 2.353 & $\begin{array}{l}\text { mean } \\
\leq 25\end{array}$ & $B=x+t s / \sqrt{n} \leq 25$ & 3.6 & pass & \multirow{2}{*}{$\begin{array}{l}\text { IRF-05B } \\
\text { DRF-26 }\end{array}$} \\
\hline & & & & 7.042 & $\begin{array}{l}\text { dispersion } \\
50.01 \geq 100\end{array}$ & $D=x+\sqrt{3} k s<100$ & 9.8 & pass & \\
\hline $\begin{array}{c}\text { Chromium content outside SiC } \\
(\mu \mathrm{g} / \text { compact })\end{array}$ & 1.58 & 0.02 & 4 & 2.353 & $\begin{array}{l}\text { mean } \\
\leq 75\end{array}$ & $B=x+t s / \sqrt{n} \leq 75$ & 1.6 & pass & $\begin{array}{l}\text { IRF-05B } \\
\text { DRF-26 }\end{array}$ \\
\hline $\begin{array}{c}\text { Manganese content outside SiC } \\
(\mu g / \text { compact })\end{array}$ & 0.42 & 0.08 & 4 & 2.353 & $\begin{array}{c}\text { mean } \\
\leq 75\end{array}$ & $B=x+t s / \sqrt{n} \leq 75$ & 0.5 & pass & $\begin{array}{l}\text { IRF-05B } \\
\text { DRF-26 }\end{array}$ \\
\hline $\begin{array}{c}\text { Cobait content outside SiC } \\
(\mu \mathrm{g} / \text { compact })\end{array}$ & 0.63 & 0.01 & 4 & 2.353 & $\begin{array}{l}\text { mean } \\
\leq 75\end{array}$ & $B=x+t s / \sqrt{n} \leq 75$ & 0.6 & pass & $\begin{array}{l}\text { IRF-05B } \\
\text { DRF-26 }\end{array}$ \\
\hline $\begin{array}{c}\text { Nickel content outside SiC } \\
(\mu g / \text { compact })\end{array}$ & 1.20 & 0.11 & 4 & 2.353 & $\begin{array}{l}\text { mean } \\
\leq 75 \\
\end{array}$ & $B=x+t s / \sqrt{n} \leq 75$ & 1.3 & pass & $\begin{array}{l}\text { IRF-05B } \\
\text { DRF-26 } \\
\end{array}$ \\
\hline $\begin{array}{c}\mathrm{Cr}+\mathrm{Mn}+\mathrm{Co}+\mathrm{Ni} \text { content outside SiC } \\
(\mu \mathrm{g} / \text { compact })\end{array}$ & 3.82 & 0.19 & 4 & 7.042 & $\begin{array}{c}\text { dispersion } \\
\leq 0.01 \geq 300 \\
\end{array}$ & $D=x+\sqrt{3} k s<300$ & 6.1 & pass & $\begin{array}{l}\text { IRF-05B } \\
\text { DRF-26 }\end{array}$ \\
\hline $\begin{array}{c}\text { Calcium content outside SiC } \\
(\mu g / \text { compact })\end{array}$ & 14.77 & 1.91 & 4 & 2.353 & $\begin{array}{l}\text { mean } \\
\leq 90\end{array}$ & $B=x+t s / \sqrt{n} \leq 90$ & 17.0 & pass & $\begin{array}{l}\text { IRF-05B } \\
\text { DRF-26 }\end{array}$ \\
\hline $\begin{array}{c}\text { Aluminum content outside SiC } \\
(\mu \mathrm{g} / \text { compact })\end{array}$ & 10.28 & 1.51 & 4 & 2.353 & $\begin{array}{l}\text { mean } \\
\leq 45 \\
\end{array}$ & $B=x+t s / \sqrt{n} \leq 45$ & 12.1 & pass & $\begin{array}{l}\text { IRF-05B } \\
\text { DRF-26 } \\
\end{array}$ \\
\hline $\begin{array}{c}\mathrm{Ti}+\mathrm{V} \text { content outside SiC } \\
(\mu \mathrm{g} / \text { compact })\end{array}$ & 27.20 & 2.33 & 4 & 2.353 & $\begin{array}{l}\text { mean } \\
\leq 400\end{array}$ & $B=x+t s / \sqrt{n} \leq 400$ & 29.9 & pass & $\begin{array}{l}\text { IRF-05B } \\
\text { DRF-26 }\end{array}$ \\
\hline
\end{tabular}

\begin{tabular}{|c|c|c|c|c|c|c|c|}
\hline \multirow[b]{2}{*}{ Property } & \multicolumn{2}{|c|}{ Measured Data } & \multirow{2}{*}{\begin{tabular}{|l|} 
Specification \\
INL EDF-4380 \\
\end{tabular}} & \multirow[b]{2}{*}{ Acceptance Criteria } & \multirow[b]{2}{*}{$\begin{array}{l}\text { Acceptance } \\
\text { Test Value }\end{array}$} & \multirow{2}{*}{$\begin{array}{l}\text { Pass } \\
\text { or } \\
\text { fall }\end{array}$} & \multirow[b]{2}{*}{$\begin{array}{c}\text { Data } \\
\text { Records }\end{array}$} \\
\hline & $\begin{array}{l}\text { *o of } \\
\text { compacts }\end{array}$ & $\begin{array}{c}\text { \# of } \\
\text { particles }\end{array}$ & & & & & \\
\hline $\begin{array}{l}\text { Uranium contamination fraction } \\
\text { ( } g \text { exposed } U / g \text { ram } U \text { in compact) }\end{array}$ & 24 & 99470 & $\leq 1.0 \times 10^{-4}$ & $\leq 4$ effectively exposed kerneis in $\geq 91533$ particles & 0 & pass & $\begin{array}{l}\text { IRF-05C } \\
\text { DRF-26 }\end{array}$ \\
\hline $\begin{array}{l}\text { Defective SiC coating fraction } \\
\text { (fraction of total particles) }\end{array}$ & 12 & 49735 & $\leq 2.0 \times 10^{-4}$ & $\begin{array}{l}\leq 4 \text { leached kernels in } 245766 \text { particles } \\
\text { or } \leq 12 \text { leached kernels in } \geq 97210 \text { particles }\end{array}$ & 2 & pass & $\begin{array}{l}\text { IRF-05D } \\
\text { DRF-26 }\end{array}$ \\
\hline $\begin{array}{l}\text { Defective IPyc coating fraction } \\
\text { (fraction of total particles) }\end{array}$ & 12 & 49735 & $\leq 2.0 \times 10^{-4}$ & $\begin{array}{l}\$ 4 \text { with excessive } U \text { dispersion in } \geq 45766 \text { particles } \\
\text { or } \$ 12 \text { with excessive } U \text { dispersion in } \geq 97210 \text { particles }\end{array}$ & 0 & pass & DRF-28 \\
\hline $\begin{array}{l}\text { Defective OPyc coating fraction } \\
\text { (fraction of total particles) }\end{array}$ & 1 & 4145 & $\leq 0.01$ & $\begin{array}{l}\leq 6 \text { cracked or missing OPyC in } \geq 1182 \text { particles } \\
\text { or } \leq 30 \text { cracked or missing OPYC in } \geq 4064 \text { particles }\end{array}$ & 0 & pass & DRF-27 \\
\hline
\end{tabular}

Comments

Note 1: 19 compacts in lot LEU01-46T-Z listed above as available for irradiation were below $25.02 \mathrm{~mm}$ in length, these were accepted for use as is per NCR-X-AGR-06-03.

Note 2: Per EDF-4380, a specification on Cl content outside SIC is not applicable because $\mathrm{HCl}$ cleaning of compacts was not performed.

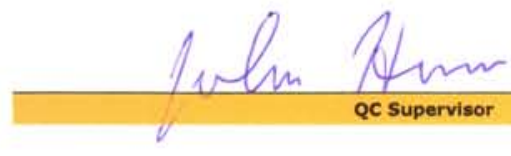

Accept compact lot (Yes or No):

\section{$7-19-06$}




\section{Inspection Report Form IRF-05B: Summary of Impurites Outside SiC}

\begin{tabular}{|r|l|}
\hline Procedure: & AGR-CHAR-PIP-05 Rev. 0 \\
\hline Operator: & Fred Montgomery \\
\hline Compact lot ID: & LEU01-46T-Z \\
\hline Compact Lot description: & Compacts of AGR-1 baseline coated particle composite LEU01-46T \\
\hline
\end{tabular}

\begin{tabular}{|c|c|c|c|c|c|c|}
\hline Compact ID numbers: & $10,13,66$ & $40,48,64$ & $35,54,59$ & $08,26,75$ & Mean & $\begin{array}{l}\text { Standard } \\
\text { Deviation }\end{array}$ \\
\hline Number of compacts: & 3 & 3 & 3 & 3 & & \\
\hline \multicolumn{7}{|l|}{ Iron } \\
\hline Deconsolidation-leach (DRF-26A) $(\mu g)$ : & 5.15 & 5.86 & 8.87 & 4.85 & & \\
\hline Burn-leach (DRF-26B) $(\mu g)$ : & 1.89 & 2.08 & 1.46 & 5.47 & & \\
\hline Total leached $(\mu \mathrm{g})$ : & 7.04 & 7.94 & 10.33 & 10.32 & & \\
\hline Fe outside SiC ( $\mu \mathrm{g} /$ compact): & 2.35 & 2.65 & 3.44 & 3.44 & 2.97 & 0.56 \\
\hline \multicolumn{7}{|l|}{ Chromium } \\
\hline Deconsolidation-leach (DRF-26A) $(\mu g)$ : & 3.36 & 3.38 & 3.36 & 3.22 & & \\
\hline Burn-leach (DRF-26B) $(\mu \mathrm{g})$ : & 1.41 & 1.41 & 1.41 & 1.41 & & \\
\hline Total leached $(\mu g)$ : & 4.77 & 4.79 & 4.77 & 4.63 & & \\
\hline Cr outside SiC ( $\mu g /$ compact): & 1.59 & 1.60 & 1.59 & 1.54 & 1.58 & 0.02 \\
\hline \multicolumn{7}{|l|}{ Manganese } \\
\hline Deconsolidation-leach (DRF-26A) $(\mu \mathrm{g})$ : & 0.85 & 0.86 & 1.19 & 1.11 & & \\
\hline Burn-leach (DRF-26B) $(\mu g)$ : & 0.20 & 0.20 & 0.37 & 0.20 & & \\
\hline Total leached $(\mu \mathrm{g})$ : & 1.05 & 1.07 & 1.56 & 1.32 & & \\
\hline Mn outside SiC ( $\mu \mathrm{g} /$ compact): & 0.35 & 0.36 & 0.52 & 0.44 & 0.42 & 0.08 \\
\hline \multicolumn{7}{|l|}{ Cobait } \\
\hline Deconsolidation-leach (DRF-26A) $(\mu g)$ : & 1.34 & 1.35 & 1.34 & 1.28 & & \\
\hline Burn-leach (DRF-26B) $(\mu g)$ : & 0.56 & 0.56 & 0.56 & 0.56 & & \\
\hline Total leached $(\mu g)$ : & 1.90 & 1.92 & 1.90 & 1.85 & & \\
\hline Co outside SiC ( $\mu \mathrm{g} /$ compact): & 0.63 & 0.64 & 0.63 & 0.62 & 0.63 & 0.01 \\
\hline \multicolumn{7}{|l|}{ Nickel } \\
\hline Deconsolidation-leach (DRF-26A) $(\mu \mathrm{g})$ : & 2.38 & 2.40 & 2.38 & 2.28 & & \\
\hline Burn-leach (DRF-26B) $(\mu \mathrm{g})$ : & 1.00 & 1.26 & 1.66 & 1.00 & & \\
\hline Total leached $(\mu g)$ : & 3.38 & 3.66 & 4.04 & 3.28 & & \\
\hline Ni outside SiC ( $\mu g /$ compact): & 1.13 & 1.22 & 1.35 & 1.09 & 1.20 & 0.11 \\
\hline \multicolumn{7}{|l|}{ Transition Metals } \\
\hline $\mathrm{Cr}+\mathrm{Mn}+\mathrm{Co}+\mathrm{Ni}$ outside SiC ( $\mu \mathrm{g} /$ compact $):$ & 3.70 & 3.81 & 4.09 & 3.69 & 3.82 & 0.19 \\
\hline \multicolumn{7}{|l|}{ Calcium } \\
\hline Deconsolidation-leach (DRF-26A) $(\mu \mathrm{g})$ : & 23.95 & 21.89 & 18.68 & 30.88 & & \\
\hline Burn-leach (DRF-26B) $(\mu g)$ : & 14.11 & 26.95 & 22.12 & 18.62 & & \\
\hline Total leached $(\mu g):$ & 38.06 & 48.84 & 40.80 & 49.50 & & \\
\hline Ca outside SiC ( $\mu \mathrm{g} /$ compact): & 12.69 & 16.28 & 13.60 & 16.50 & 14.77 & 1.91 \\
\hline \multicolumn{7}{|l|}{ Aluminum } \\
\hline Deconsolidation-leach (DRF-26A) $(\mu \mathrm{g})$ : & 19.53 & 18.16 & 15.96 & 27.00 & & \\
\hline Burn-leach (DRF-26B) $(\mu g)$ : & 14.13 & 14.83 & 8.14 & 5.62 & & \\
\hline Total leached $(\mu g)$ : & 33.66 & 32.99 & 24.10 & 32.62 & & \\
\hline Al outside SiC ( $\mu \mathrm{g} /$ compact): & 11.22 & 11.00 & 8.03 & 10.87 & 10.28 & 1.51 \\
\hline \multicolumn{7}{|l|}{ Titanium } \\
\hline Deconsolidation-leach (DRF-26A) $(\mu g)$ : & 17.01 & 14.58 & 14.90 & 13.46 & & \\
\hline Burn-leach (DRF-26B) $(\mu \mathrm{g})$ : & 4.96 & 12.76 & 14.09 & 16.26 & & \\
\hline Total leached $(\mu g)$ : & 21.97 & 27.34 & 28.99 & 29.72 & & \\
\hline Ti outside SiC ( $\mu \mathrm{g} /$ compact): & 7.32 & 9.11 & 9.66 & 9.91 & 9.00 & 1.17 \\
\hline \multicolumn{7}{|l|}{ Vanadium } \\
\hline Deconsolidation-leach (DRF-26A) $(\mu \mathrm{g})$ : & 29.60 & 28.43 & 30.98 & 30.26 & & \\
\hline Burn-leach (DRF-26B) $(\mu \mathrm{g})$ : & 22.51 & 21.42 & 27.54 & 27.60 & & \\
\hline Total leached $(\mu g)$ : & 52.11 & 49.85 & 58.52 & 57.86 & & \\
\hline V outside SiC ( $\mu \mathrm{g} /$ compact): & 17.37 & 16.62 & 19.51 & 19.29 & 18.20 & 1.42 \\
\hline \multicolumn{7}{|l|}{ Titanium and Vanadium } \\
\hline $\mathrm{Ti}+\mathrm{V}$ outside SiC ( $\mu \mathrm{g} /$ compact): & 24.69 & 25.73 & 29.17 & 29.19 & 27.20 & 2.33 \\
\hline
\end{tabular}
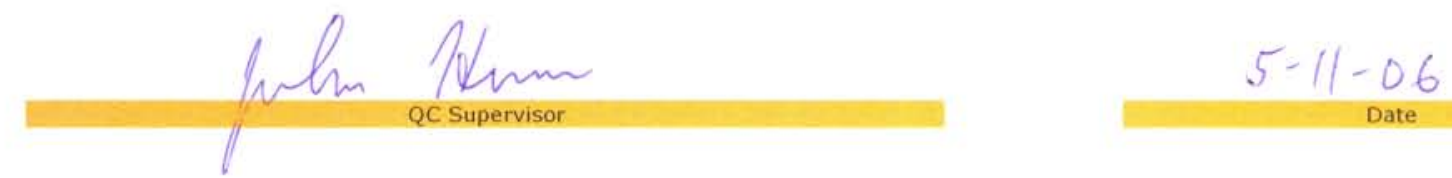
ORNL/TM-2006/507, Rev. 0

Inspection Report Form IRF-05C: Summary of Uranium Contamination

Procedure: AGR-CHAR-PIP-05 Rev. 0

Operator: Fred Montgomery

Compact lot ID: LEU01-46T-Z

Compact Lot description: Compacts of AGR-1 baseline coated particle composite LEU01-46T

\begin{tabular}{|r|c|c|c|c|c|}
\hline Compact ID numbers: & $10,13,66$ & $40,48,64$ & $35,54,59$ & $08,26,75$ & $\begin{array}{c}02,04,11,28, \\
31,61\end{array}$ \\
\hline Number of compacts: & 3 & 3 & 3 & 3 & 6 \\
\hline Effective number of exposed kernels: & 0.0 & 0.0 & 0.0 & 0.0 & 6 \\
\hline
\end{tabular}

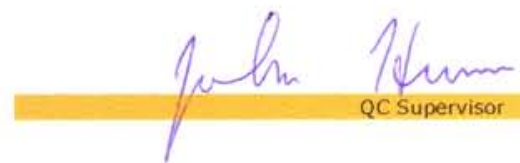

$$
5-11-06
$$


ORNL/TM-2006/507, Rev. 0

\section{Inspection Report Form IRF-05D: Summary of SiC Burn-Leach Defects}

Procedure: AGR-CHAR-PIP-05 Rev. 0

Operator: Fred Montgomery

Compact lot ID: LEU01-46T-Z

Compact Lot description: Compacts of AGR-1 baseline coated particle composite LEU01-46T

\begin{tabular}{|r|c|c|c|c|c|c|}
\hline Compact ID numbers: & $10,13,66$ & $40,48,64$ & $35,54,59$ & $08,26,75$ & Total \\
\hline \hline Number of compacts: & 3 & 3 & 3 & 3 & & 12 \\
\hline Number of leached kernels: & 1 & 1 & 0 & 0 & 12 \\
\hline
\end{tabular}

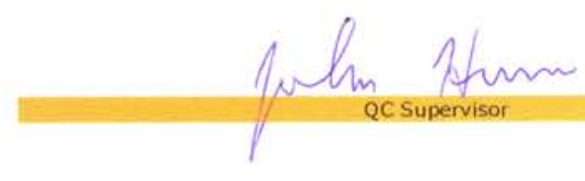

$$
5-11-06
$$




\section{Compacting process conditions}

LEU01-46T TRISO (baseline) particles were received from the characterization group after removing particles for characterization according to AGR-CHAR-PIP-04R2, "Product Inspection Plan for Coated Particle Composites." Nineteen 20 g aliquots were prepared via riffling, and one aliquot was used per overcoating run. Prior to overcoating, the particles were washed in methanol per procedure AGR-TRISOWASH-SOP-1, "Standard Operating Procedure for TRISO Particle Washing." Washing of particles prior to overcoating was adapted in order to help reduce the amount of contamination on the particles that may have been acquired during processing or general handling. The washing procedure was adopted from General Atomics' particle washing procedures.

After washing, the LEU01-46T particles were overcoated using matrix batch GKrS 121405. All of the aliquots were overcoated producing $396 \mathrm{~g}$ of +18 particles. " +18 " particles are those that pass through an ASTM E11 No. 16 sieve $(1.18 \mathrm{~mm})$ but do not pass through and ASTM E11 No. 18 sieve $(1.00 \mathrm{~mm})$. This quantity of +18 particles was insufficient (based on assumed weight per overcoated particle data and past overcoating experience) to produce 79 compacts, so -18 overcoated particles (those particles that passed through an ASTM E11 No. 18 sieve) were rinsed with methanol in order to remove the overcoat, and subsequently re-overcoated. Prior to rinsing off the overcoat, overcoating of the -18 particles was performed in an attempt to increase the overcoat thickness enough such that +18 overcoated particles would be produced. However, the +18 overcoated particles achieved in this re-overcoating manner were different in color than the +18 overcoated particles achieved in a standard overcoating run, and were therefore considered undesirable. Overcoated particle color is not specified in the overcoating procedure, but the PI decided it was better to overcoat all the TRISO particles in the same manner in order to produce overcoated particles with properties as similar as possible. A standard overcoating run is when TRISO particles (as opposed to -18 particles that have already been partially overcoated) are overcoated until +18 particles are achieved. Overcoating of reclaimed TRISO particles (TRISO particles from rinsed overcoated particles) was continued until $537 \mathrm{~g}$ of +18 particles was produced.

The $537 \mathrm{~g}$ of +18 particles was then tabled and $410 \mathrm{~g}$ of +18 Bin 3 particles were recovered. "Bin 3" particles are those particles that end up in the third bin of the tabler; these are the most spherical of the +18 particles. $410 \mathrm{~g}$ of +18 Bin 3 overcoated particles was determined to be a sufficient quantity to produce at least 79 compacts, based on preliminary calculations.

Based on an average kernel weight of $2.42 \cdot 10^{-4} \mathrm{~g}$ and a wt $\%$ uranium of 0.9006 for the AGR-1 kernels, 4151 particles are needed in each compact to obtain a uranium loading of $0.905 \mathrm{~g}$. The average +18 Bin 3 overcoated particle weight was measured (according to AGR-CHAR-DAM22, "Data Acquisition Method for Estimation of Average Particle Weight") to be $1.16 \cdot 10^{-3} \mathrm{~g}$. Using this value, a compact charge of $4.82 \mathrm{~g}$ of +18 Bin 3 overcoated particles would be required for a compact uranium loading of $0.905 \mathrm{~g}$. As an alternate approach for calculating the compact charge, a quantity of +18 Bin 3 overcoated particles was rinsed and the ratio of overcoated particle weight to TRISO particle weight was determined to be 1.613.4151 particles corresponds 
to $3.018 \mathrm{~g}$ TRISO particles (given an average weight for AGR-1 baseline TRISO particles of $7.27 \cdot 10^{-4} \mathrm{~g}$ ). Using this second approach, a compact charge of $4.87 \mathrm{~g}$ of +18 Bin 3 overcoated particles would be required for a compact uranium loading of $0.905 \mathrm{~g}$. Based on these two determinations, an overcoated particle charge of $4.86 \mathrm{~g}$ was used to increase the probability that the actual uranium loading would be $\geq 0.905 \mathrm{~g}$.

The 410 g quantity of +18 Bin 3 overcoated particles was rotary riffled into aliquots of approximately $4.50 \mathrm{~g}$. Additional +18 Bin 3 overcoated particles were added to each aliquot by scoop sampling until the desired $4.86 \mathrm{~g}$ compact charge was reached. Compacts were then made from the $4.86 \mathrm{~g}$ aliquots. Steps were taken in the process to increase the malleability of the overcoat, which helped aid the compaction process, densify the compact, and create a smoother, less porous outer surface. A $0.40 \mathrm{~g}$ quantity of matrix powder was added to the compacting mold prior to the addition of overcoated particles. Another $0.40 \mathrm{~g}$ quantity of matrix was added to the mold after addition of the overcoated particles, such that the overcoated particles were compressed between two matrix "end caps" during fabrication. The compact is pressed by applying force to a top ram. The presence of the end caps helps to buffer the particles during compacting, and also provides a smooth, sharp end in the green compact. The thickness of the end caps is difficult to determine because a cylindrical geometry is not likely. Overcoat matrix and end cap matrix can be expected to intertwine and particles may extend beyond the apparent end cap boundary evident at the compact surface. The top end cap thickness was estimated by measuring the approximate width of the unfueled region that could be observed on the compact surface. The average top end cap thickness for 5 compacts estimated by this method was 1.3-1.5 $\mathrm{mm}$. The bottom end cap was not measured, but was slightly thicker than the top end cap.

84 AGR-1 baseline compacts were fabricated. All of the 84 green compacts were subsequently carbonized and heat treated. 79 compacts were selected from this batch of 84 and delivered to the characterization group. The selection of the 79 compacts for the characterization group was based on length measurements at each stage of compacting (green, carbonization, and heattreatment), and visual inspection for surface irregularities.

\section{AGR-1 Process Conditions}

The AGR-1 process limits from EDF-4380, Rev. 8 are listed below.

AGR-1 Process Limits: $\quad$ Molding Pressure $<60 \mathrm{MPa}$

Carbonization parameters: $<350^{\circ} \mathrm{C} / \mathrm{hr}$ in $\mathrm{He}$

Hold at $950 \pm 50^{\circ} \mathrm{C}$ for $1.0 \pm 0.4 \mathrm{hr}$

Furnace cool

Heat treatment parameters: $\sim 20^{\circ} \mathrm{C} / \mathrm{min}$ in vacuum

Hold at $1650-1850^{\circ} \mathrm{C}$ for $60 \pm 10 \mathrm{~min}$

Furnace cool at $\sim 20^{\circ} \mathrm{C} / \mathrm{min}$ to below $700^{\circ} \mathrm{C}$ 
Table 2-1 shows the process conditions used in molding the compacts, carbonizing the compacts, and heat treating the compacts. In the carbonization regime, the furnace was allowed to cool under no power (i.e., after holding at $950^{\circ} \mathrm{C}$ for 1 hour, power was turned off). In the heat treatment run, the furnace was cooled under power until the furnace temperature reached $700^{\circ} \mathrm{C}$. The rate of cooling was $20^{\circ} \mathrm{C} / \mathrm{min}$.

\section{Conclusion}

The LEU01-46T-Z (AGR-1 baseline) compact lot was made in accordance with the AGR-1 process limits listed in EDF-4380, Rev. 8. 
Table 2-1: Summary of process conditions used in making LEU01-46T-Z (AGR-1 baseline) compacts

\begin{tabular}{|c|c|c|c|c|c|c|c|c|c|}
\hline \multirow[b]{2}{*}{ Compact ID } & \multirow[b]{2}{*}{$\begin{array}{c}\text { Molding Pressure } \\
\text { (MPa) }\end{array}$} & \multicolumn{4}{|c|}{ Carbonization Parameter } & \multicolumn{4}{|c|}{ Heat-treatment Parameters } \\
\hline & & $\begin{array}{l}\text { Heating Rate } \\
\left({ }^{\circ} \mathrm{C} / \mathrm{min} .\right)\end{array}$ & $\begin{array}{l}\text { Max. Temp. } \\
\left({ }^{\circ} \mathrm{C}\right)\end{array}$ & $\begin{array}{l}\text { Hold Time } \\
\text { (hrs.) }\end{array}$ & Atmosphere & $\begin{array}{l}\text { Heating Rate } \\
\left({ }^{\circ} \mathrm{C} / \mathrm{min} .\right)\end{array}$ & $\begin{array}{l}\text { Max. Temp. } \\
\left({ }^{\circ} \mathrm{C}\right)\end{array}$ & $\begin{array}{l}\text { Hold Time } \\
\text { (hrs.) }\end{array}$ & Atmosphere \\
\hline LEU01-46T-Z01 & 13.92 & 4.7 & 950 & 1 & flowing $\mathrm{He}$ & 20 & 1800 & 1 & vacuum \\
\hline LEU01-46T-Z02 & 14.85 & 4.7 & 950 & 1 & flowing $\mathrm{He}$ & 20 & 1800 & 1 & vacuum \\
\hline LEU01-46T-ZO3 & 22.28 & 4.7 & 950 & 1 & flowing $\mathrm{He}$ & 20 & 1800 & 1 & vacuum \\
\hline LEU01-46T-ZO4 & 13.00 & 4.7 & 950 & 1 & flowing $\mathrm{He}$ & 20 & 1800 & 1 & vacuum \\
\hline LEU01-46T-ZO5 & 11.14 & 4.7 & 950 & 1 & flowing $\mathrm{He}$ & 20 & 1800 & 1 & vacuum \\
\hline LEU01-46T-Z06 & 14.85 & 4.7 & 950 & 1 & flowing $\mathrm{He}$ & 20 & 1800 & 1 & vacuum \\
\hline LEU01-46T-ZO7 & 14.85 & 4.7 & 950 & 1 & flowing $\mathrm{He}$ & 20 & 1800 & 1 & vacuum \\
\hline LEU01-46T-Z08 & 14.85 & 4.7 & 950 & 1 & flowing $\mathrm{He}$ & 20 & 1800 & 1 & vacuum \\
\hline LEU01-46T-Z09 & 13.92 & 4.7 & 950 & 1 & flowing $\mathrm{He}$ & 20 & 1800 & 1 & vacuum \\
\hline LEU01-46T-Z10 & 14.85 & 4.7 & 950 & 1 & flowing $\mathrm{He}$ & 20 & 1800 & 1 & vacuum \\
\hline LEU01-46T-Z11 & 13.00 & 4.7 & 950 & 1 & flowing $\mathrm{He}$ & 20 & 1800 & 1 & vacuum \\
\hline LEU01-46T-Z12 & 13.92 & 4.7 & 950 & 1 & flowing $\mathrm{He}$ & 20 & 1800 & 1 & vacuum \\
\hline LEU01-46T-Z13 & 14.85 & 4.7 & 950 & 1 & flowing $\mathrm{He}$ & 20 & 1800 & 1 & vacuum \\
\hline LEU01-46T-Z14 & 13.92 & 4.7 & 950 & 1 & flowing $\mathrm{He}$ & 20 & 1800 & 1 & vacuum \\
\hline LEU01-46T-Z15 & 14.85 & 4.7 & 950 & 1 & flowing $\mathrm{He}$ & 20 & 1800 & 1 & vacuum \\
\hline LEU01-46T-Z16 & 14.85 & 4.7 & 950 & 1 & flowing $\mathrm{He}$ & 20 & 1800 & 1 & vacuum \\
\hline LEU01-46T-Z17 & 14.85 & 4.7 & 950 & 1 & flowing $\mathrm{He}$ & 20 & 1800 & 1 & vacuum \\
\hline LEU01-46T-Z18 & 14.85 & 4.7 & 950 & 1 & flowing $\mathrm{He}$ & 20 & 1800 & 1 & vacuum \\
\hline LEU01-46T-Z19 & 11.14 & 4.7 & 950 & 1 & flowing $\mathrm{He}$ & 20 & 1800 & 1 & vacuum \\
\hline LEU01-46T-Z20 & 15.78 & 4.7 & 950 & 1 & flowing $\mathrm{He}$ & 20 & 1800 & 1 & vacuum \\
\hline LEU01-46T-Z21 & 15.78 & 4.7 & 950 & 1 & flowing $\mathrm{He}$ & 20 & 1800 & 1 & vacuum \\
\hline LEU01-46T-ZZ2 & 14.85 & 4.7 & 950 & 1 & flowing $\mathrm{He}$ & 20 & 1800 & 1 & vacuum \\
\hline LEU01-46T-Z23 & 14.85 & 4.7 & 950 & 1 & flowing $\mathrm{He}$ & 20 & 1800 & 1 & vacuum \\
\hline LEU01-46T-Z224 & 14.85 & 4.7 & 950 & 1 & flowing $\mathrm{He}$ & 20 & 1800 & 1 & vacuum \\
\hline LEU01-46T-Z25 & 13.92 & 4.7 & 950 & 1 & flowing $\mathrm{He}$ & 20 & 1800 & 1 & vacuum \\
\hline LEU01-46T-Z26 & 14.85 & 4.7 & 950 & 1 & flowing $\mathrm{He}$ & 20 & 1800 & 1 & vacuum \\
\hline LEU01-46T-Z27 & 14.85 & 4.7 & 950 & 1 & flowing $\mathrm{He}$ & 20 & 1800 & 1 & vacuum \\
\hline LEU01-46T-Z28 & 12.07 & 4.7 & 950 & 1 & flowing $\mathrm{He}$ & 20 & 1800 & 1 & vacuum \\
\hline LEU01-46T-Z29 & 13.92 & 4.7 & 950 & 1 & flowing $\mathrm{He}$ & 20 & 1800 & 1 & vacuum \\
\hline LEU01-46T-Z30 & 12.07 & 4.7 & 950 & 1 & flowing $\mathrm{He}$ & 20 & 1800 & 1 & vacuum \\
\hline LEU01-46T-Z31 & 14.85 & 4.7 & 950 & 1 & flowing $\mathrm{He}$ & 20 & 1800 & 1 & vacuum \\
\hline LEU01-46T-Z32 & 14.85 & 4.7 & 950 & 1 & flowing $\mathrm{He}$ & 20 & 1800 & 1 & vacuum \\
\hline LEU01-46T-Z33 & 14.85 & 4.7 & 950 & 1 & flowing $\mathrm{He}$ & 20 & 1800 & 1 & vacuum \\
\hline LEU01-46T-Z34 & 14.85 & 4.7 & 950 & 1 & flowing $\mathrm{He}$ & 20 & 1800 & 1 & vacuum \\
\hline LEU01-46T-Z35 & 13.92 & 4.7 & 950 & 1 & flowing $\mathrm{He}$ & 20 & 1800 & 1 & vacuum \\
\hline LEU01-46T-Z36 & 13.92 & 4.7 & 950 & 1 & flowing $\mathrm{He}$ & 20 & 1800 & 1 & vacuum \\
\hline
\end{tabular}

Task Manager Review leter J.?

Date $8-1-06$

QAS Review

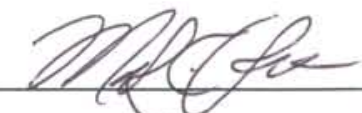

Date $8 / 1 / 06$ 
Table 2-1 (cont.). Summary of process conditions used in making LEU01-46T-Z (AGR-1 baseline) compacts

\begin{tabular}{|c|c|c|c|c|c|c|c|c|c|}
\hline \multicolumn{6}{|c|}{ Carbonization Parameter } & \multicolumn{4}{|c|}{ Heat-treatment Parameters } \\
\hline Compact ID & $\begin{array}{c}\text { Molding Pressure } \\
(\mathrm{MPa})\end{array}$ & $\begin{array}{l}\text { Heating Rate } \\
\left({ }^{\circ} \mathrm{C} / \mathrm{min} .\right)\end{array}$ & $\begin{array}{l}\text { Max. Temp. } \\
\left({ }^{\circ} \mathrm{C}\right)\end{array}$ & $\begin{array}{l}\text { Hold Time } \\
\text { (hrs.) }\end{array}$ & Atmosphere & $\begin{array}{l}\text { Heating Rate } \\
\left({ }^{\circ} \mathrm{C} / \mathrm{min} .\right)\end{array}$ & $\begin{array}{l}\text { Max. Temp. } \\
\left({ }^{\circ} \mathrm{C}\right)\end{array}$ & $\begin{array}{l}\text { Hold Time } \\
\text { (hrs.) }\end{array}$ & Atmosphere \\
\hline LEU01-46T-Z37 & 14.85 & 4.7 & 950 & 1 & flowing $\mathrm{He}$ & 20 & 1800 & 1 & vacuum \\
\hline LEU01-46T-Z38 & 29.71 & 4.7 & 950 & 1 & flowing $\mathrm{He}$ & 20 & 1800 & 1 & vacuum \\
\hline LEU01-46T-Z39 & 12.07 & 4.7 & 950 & 1 & flowing $\mathrm{He}$ & 20 & 1800 & 1 & vacuum \\
\hline LEU01-46T-Z40 & 14.85 & 4.7 & 950 & 1 & flowing $\mathrm{He}$ & 20 & 1800 & 1 & vacuum \\
\hline LEU01-46T-Z41 & 14.85 & 4.7 & 950 & 1 & flowing $\mathrm{He}$ & 20 & 1800 & 1 & vacuum \\
\hline LEU01-46T-Z42 & 13.92 & 4.7 & 950 & 1 & flowing $\mathrm{He}$ & 20 & 1800 & 1 & vacuum \\
\hline LEU01-46T-Z43 & 12.07 & 4.7 & 950 & 1 & flowing $\mathrm{He}$ & 20 & 1800 & 1 & vacuum \\
\hline LEU01-46T-Z44 & 13.92 & 4.7 & 950 & 1 & flowing $\mathrm{He}$ & 20 & 1800 & 1 & vacuum \\
\hline LEU01-46T-Z45 & 13.92 & 4.7 & 950 & 1 & flowing $\mathrm{He}$ & 20 & 1800 & 1 & vacuum \\
\hline LEU01-46T-Z46 & 13.00 & 4.7 & 950 & 1 & flowing $\mathrm{He}$ & 20 & 1800 & 1 & vacuum \\
\hline LEU01-46T-Z47 & 14.85 & 4.7 & 950 & 1 & flowing $\mathrm{He}$ & 20 & 1800 & 1 & vacuum \\
\hline LEU01-46T-Z48 & 16.71 & 4.7 & 950 & 1 & flowing $\mathrm{He}$ & 20 & 1800 & 1 & vacuum \\
\hline LEU01-46T-Z49 & 14.85 & 4.7 & 950 & 1 & flowing $\mathrm{He}$ & 20 & 1800 & 1 & vacuum \\
\hline LEU01-46T-Z50 & 13.00 & 4.7 & 950 & 1 & flowing $\mathrm{He}$ & 20 & 1800 & 1 & vacuum \\
\hline LEU01-46T-Z51 & 13.00 & 4.7 & 950 & 1 & flowing $\mathrm{He}$ & 20 & 1800 & 1 & vacuum \\
\hline LEU01-46T-Z52 & 13.00 & 4.7 & 950 & 1 & flowing $\mathrm{He}$ & 20 & 1800 & 1 & vacuum \\
\hline LEU01-46T-Z53 & 14.85 & 4.7 & 950 & 1 & flowing $\mathrm{He}$ & 20 & 1800 & 1 & vacuum \\
\hline LEU01-46T-Z54 & 14.85 & 4.7 & 950 & 1 & flowing $\mathrm{He}$ & 20 & 1800 & 1 & vacuum \\
\hline LEU01-46T-Z55 & 13.00 & 4.7 & 950 & 1 & flowing $\mathrm{He}$ & 20 & 1800 & 1 & vacuum \\
\hline LEU01-46T-Z56 & 14.85 & 4.7 & 950 & 1 & flowing $\mathrm{He}$ & 20 & 1800 & 1 & vacuum \\
\hline LEU01-46T-Z57 & 13.00 & 4.7 & 950 & 1 & flowing $\mathrm{He}$ & 20 & 1800 & 1 & vacuum \\
\hline LEU01-46T-Z58 & 13.00 & 4.7 & 950 & 1 & flowing $\mathrm{He}$ & 20 & 1800 & 1 & vacuum \\
\hline LEU01-46T-Z59 & 13.00 & 4.7 & 950 & 1 & flowing $\mathrm{He}$ & 20 & 1800 & 1 & vacuum \\
\hline LEU01-46T-Z60 & 11.14 & 4.7 & 950 & 1 & flowing $\mathrm{He}$ & 20 & 1800 & 1 & vacuum \\
\hline LEU01-46T-Z61 & 13.92 & 4.7 & 950 & 1 & flowing $\mathrm{He}$ & 20 & 1800 & 1 & vacuum \\
\hline LEU01-46T-Z62 & 13.00 & 4.7 & 950 & 1 & flowing $\mathrm{He}$ & 20 & 1800 & 1 & vacuum \\
\hline LEU01-46T-Z63 & 14.85 & 4.7 & 950 & 1 & flowing $\mathrm{He}$ & 20 & 1800 & 1 & vacuum \\
\hline LEU01-46T-Z64 & 16.71 & 4.7 & 950 & 1 & flowing $\mathrm{He}$ & 20 & 1800 & 1 & vacuum \\
\hline LEU01-46T-Z65 & 14.85 & 4.7 & 950 & 1 & flowing $\mathrm{He}$ & 20 & 1800 & 1 & vacuum \\
\hline LEU01-46T-Z66 & 13.92 & 4.7 & 950 & 1 & flowing $\mathrm{He}$ & 20 & 1800 & 1 & vacuum \\
\hline LEU01-46T-Z67 & 13.00 & 4.7 & 950 & 1 & flowing $\mathrm{He}$ & 20 & 1800 & 1 & vacuum \\
\hline LEU01-46T-Z68 & 14.85 & 4.7 & 950 & 1 & flowing $\mathrm{He}$ & 20 & 1800 & 1 & vacuum \\
\hline LEU01-46T-Z69 & 13.92 & 4.7 & 950 & 1 & flowing $\mathrm{He}$ & 20 & 1800 & 1 & vacuum \\
\hline LEU01-46T-Z70 & 14.85 & 4.7 & 950 & 1 & flowing $\mathrm{He}$ & 20 & 1800 & 1 & vacuum \\
\hline LEU01-46T-Z71 & 14.85 & 4.7 & 950 & 1 & flowing $\mathrm{He}$ & 20 & 1800 & 1 & vacuum \\
\hline LEU01-46T-Z72 & 13.92 & 4.7 & 950 & 1 & flowing $\mathrm{He}$ & 20 & 1800 & 1 & vacuum \\
\hline
\end{tabular}

Task Manager Review Petv J. Pappaner

Date $8-1-06$

QAS Review

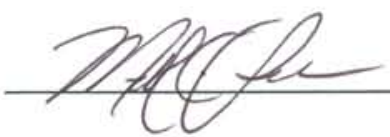

Date $8 / 1 / 06$ 
Table 2-1 (cont.). Summary of process conditions used in making LEU01-46T-Z (AGR-1 baseline) compacts

\begin{tabular}{|c|c|c|c|c|c|c|c|c|c|}
\hline & & \multicolumn{4}{|c|}{ Carbonization Parameter } & \multicolumn{4}{|c|}{ Heat-treatment Parameters } \\
\hline Compact ID & $\begin{array}{l}\text { Molding Pressure } \\
\text { (MPa) }\end{array}$ & $\begin{array}{l}\text { Heating Rate } \\
\left({ }^{\circ} \mathrm{C} / \mathrm{min} .\right)\end{array}$ & $\begin{array}{l}\text { Max. Temp. } \\
\left({ }^{\circ} \mathrm{C}\right)\end{array}$ & $\begin{array}{l}\text { Hold Time } \\
\text { (hrs.) }\end{array}$ & Atmosphere & $\begin{array}{l}\text { Heating Rate } \\
\left({ }^{\circ} \mathrm{C} / \mathrm{min} .\right)\end{array}$ & $\begin{array}{l}\text { Max. Temp. } \\
\left({ }^{\circ} \mathrm{C}\right)\end{array}$ & $\begin{array}{l}\text { Hold Time } \\
\text { (hrs.) }\end{array}$ & Atmosphere \\
\hline LEU01-46T-Z73 & 11.14 & 4.7 & 950 & 1 & flowing $\mathrm{He}$ & 20 & 1800 & 1 & vacuum \\
\hline LEU01-46T-Z74 & 15.78 & 4.7 & 950 & 1 & flowing $\mathrm{He}$ & 20 & 1800 & 1 & vacuum \\
\hline LEU01-46T-Z75 & 14.85 & 4.7 & 950 & 1 & flowing $\mathrm{He}$ & 20 & 1800 & 1 & vacuum \\
\hline LEU01-46T-Z76 & 13.92 & 4.7 & 950 & 1 & flowing $\mathrm{He}$ & 20 & 1800 & 1 & vacuum \\
\hline LEU01-46T-Z77 & 13.00 & 4.7 & 950 & 1 & flowing $\mathrm{He}$ & 20 & 1800 & 1 & vacuum \\
\hline LEU01-46T-Z78 & 13.92 & 4.7 & 950 & 1 & flowing $\mathrm{He}$ & 20 & 1800 & 1 & vacuum \\
\hline LEU01-46T-Z79 & 13.92 & 4.7 & 950 & 1 & flowing $\mathrm{He}$ & 20 & 1800 & 1 & vacuum \\
\hline
\end{tabular}

Task Manager Review Reter I Pyen

Date $8-1-06$

QAS Review

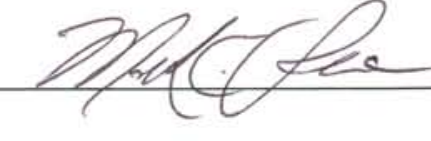

Date $8 / 1 / 06$ 


\section{Characterization of compacts}

This section contains acceptance testing data on the compact lot LEU01-46T-Z. The data was obtained according to product inspection plan AGR-CHAR-PIP-05R0.

The following pages show the inspection report forms (IRF-05A, IRF-05B, IRF-05C, IRF-05D) for the LEU01-46T-Z compacts. Following the IRF-05 inspection report forms are the individual data report forms for the measurements that were performed. This compact lot was determined to satisfy the specifications in section 6.2 of the AGR-1 Fuel Product Specification and Characterization Guidance document INL EDF-4380, Rev. 8, with the exception of 19 compacts which were available for irradiation that did not meet the specified minimum length. These 19 non-conforming compacts have been dispositioned for use as is by NCR-X-AGR-06-03. 


\begin{tabular}{|c|c|c|c|c|c|c|c|c|c|}
\hline \multirow[b]{2}{*}{ Property } & \multicolumn{4}{|c|}{ Measured Data } & \multirow{2}{*}{$\begin{array}{c}\text { Specification } \\
\text { INL EDF- } 4380 \\
\text { Rev, } 8\end{array}$} & \multirow[b]{2}{*}{ Acceptance Criteria } & \multirow[b]{2}{*}{$\begin{array}{l}\text { Acceptance } \\
\text { Test Value }\end{array}$} & \multirow{2}{*}{$\begin{array}{l}\text { Pass } \\
\text { or } \\
\text { fail }\end{array}$} & \multirow[b]{2}{*}{$\begin{array}{c}\text { Data } \\
\text { Records }\end{array}$} \\
\hline & $\begin{array}{c}\text { Mean } \\
(x)\end{array}$ & $\begin{array}{l}\text { Std. Dev. } \\
\text { (s) }\end{array}$ & $\begin{array}{c}\text { Measurements } \\
(n)\end{array}$ & $\begin{array}{l}k \text { or } t \\
\text { value }\end{array}$ & & & & & \\
\hline Compact diameter $(\mathrm{mm})$ & \multirow{2}{*}{\multicolumn{2}{|c|}{ See DRF-24 }} & & & $12.22-12.46$ & all available for irradiation test & & & DRE-24 \\
\hline Compact length $(\mathrm{mm})$ & & & & & $25.02-25.40$ & meet specification & & (Note 1) & $D k r=24$ \\
\hline \multirow{2}{*}{$\begin{array}{l}\text { Uranium loading } \\
\text { ( } \mathrm{U} \text { /compact) }\end{array}$} & \multirow{2}{*}{0.917} & \multirow{2}{*}{0.005} & \multirow{2}{*}{6} & \multirow{2}{*}{2.015} & \multirow{2}{*}{$0.905 \pm 0.04$} & $A=x-t s / \sqrt{ } n \geq 0.865$ & 0.913 & pass & \multirow{2}{*}{ DRF-25 } \\
\hline & & & & & & $B=x+t s / \sqrt{n} \leq 0.945$ & 0.921 & pass & \\
\hline \multirow{2}{*}{$\begin{array}{l}\text { Iron content outside SiC } \\
\text { ( } \mu \mathrm{g} / \text { compact) }\end{array}$} & \multirow{2}{*}{2.97} & \multirow{2}{*}{0.56} & \multirow{2}{*}{4} & 2.353 & $\begin{array}{l}\text { mean } \\
\leq 25\end{array}$ & $B=x+t s / \sqrt{n} \leq 25$ & 3.6 & pass & \multirow{2}{*}{$\begin{array}{l}\text { IRF-05B } \\
\text { DRF-26 }\end{array}$} \\
\hline & & & & 7.042 & $\begin{array}{c}\text { dispersion } \\
\leq 0.01 \geq 100\end{array}$ & $D=x+\sqrt{3} k s<100$ & 9.8 & pass & \\
\hline $\begin{array}{c}\text { Chromium content outside SiC } \\
(\mu g / \text { compact })\end{array}$ & 1.58 & 0.02 & 4 & 2.353 & $\begin{array}{l}\text { mean } \\
\leq 75 \\
\end{array}$ & $B=x+t s / \sqrt{ } n \leq 75$ & 1.6 & pass & $\begin{array}{l}\text { IRF-05B } \\
\text { DRF-26 }\end{array}$ \\
\hline $\begin{array}{c}\text { Manganese content outside SiC } \\
(\mu \mathrm{g} / \mathrm{compact})\end{array}$ & 0.42 & 0.08 & 4 & 2.353 & $\begin{array}{l}\text { mean } \\
\leq 75\end{array}$ & $B=x+t s / \sqrt{ } n \leq 75$ & 0.5 & pass & $\begin{array}{l}\text { IRF-05B } \\
\text { DRF-26 }\end{array}$ \\
\hline $\begin{array}{c}\text { Cobalt content outside SiC } \\
\text { ( } \mu \mathrm{g} / \text { compact })\end{array}$ & 0.63 & 0.01 & 4 & 2.353 & $\begin{array}{l}\text { mean } \\
\leq 75\end{array}$ & $B=x+t s / \sqrt{ } n \leq 75$ & 0.6 & pass & $\begin{array}{l}\text { IRF-05B } \\
\text { DRF-26 }\end{array}$ \\
\hline $\begin{array}{c}\text { Nickel content outside SiC } \\
\text { ( } \mu \mathrm{g} / \text { compact) }\end{array}$ & 1.20 & 0.11 & 4 & 2.353 & $\begin{array}{l}\text { mean } \\
\leq 75\end{array}$ & $B=x+t s / \sqrt{ } n \leq 75$ & 1.3 & pass & $\begin{array}{l}\text { IRF-05B } \\
\text { DRF-26 }\end{array}$ \\
\hline $\begin{array}{c}\mathrm{Cr}+\mathrm{Mn}+\mathrm{Co}+\mathrm{Ni} \text { content outside SiC } \\
(\mu \mathrm{g} / \text { compact })\end{array}$ & 3.82 & 0.19 & 4 & 7.042 & $\begin{array}{l}\text { dispersion } \\
\leq 0.01 \geq 300\end{array}$ & $D=x+\sqrt{3} k s<300$ & 6.1 & pass & $\begin{array}{l}\text { IRF-05B } \\
\text { DRF-26 }\end{array}$ \\
\hline $\begin{array}{c}\text { Calcium content outside SiC } \\
(\mu g / \text { compact })\end{array}$ & 14.77 & 1.91 & 4 & 2.353 & $\begin{array}{c}\text { mean } \\
\leq 90\end{array}$ & $B=x+t s / \sqrt{ } n \leq 90$ & 17.0 & pass & $\begin{array}{l}\text { IRF-05B } \\
\text { DRF-26 }\end{array}$ \\
\hline $\begin{array}{c}\text { Aluminum content outside SiC } \\
(\mu \mathrm{g} / \text { compact })\end{array}$ & 10.28 & 1.51 & 4 & 2.353 & $\begin{array}{l}\text { mean } \\
\leq 45\end{array}$ & $B=x+t s / \sqrt{ } n \leq 45$ & 12.1 & pass & $\begin{array}{l}\text { IRF-05B } \\
\text { DRF-26 }\end{array}$ \\
\hline $\begin{array}{c}\mathrm{Ti}+\mathrm{V} \text { content outside SiC } \\
(\mu \mathrm{g} / \text { compact })\end{array}$ & 27.20 & 2.33 & 4 & 2.353 & $\begin{array}{l}\text { mean } \\
\leq 400\end{array}$ & $B=x+t s / \sqrt{n} \leq 400$ & 29.9 & pass & $\begin{array}{l}\text { IRF-05B } \\
\text { DRF-26 }\end{array}$ \\
\hline
\end{tabular}

\begin{tabular}{|c|c|c|c|c|c|c|c|}
\hline \multirow[b]{2}{*}{ Property } & \multicolumn{2}{|c|}{ Measured Data } & \multirow{2}{*}{$\begin{array}{c}\text { Specification } \\
\text { INL EDF- } 4380\end{array}$} & \multirow[b]{2}{*}{ Acceptance Criteria } & \multirow[b]{2}{*}{$\begin{array}{l}\text { Acceptance } \\
\text { Test Value }\end{array}$} & \multirow{2}{*}{$\begin{array}{l}\text { Pass } \\
\text { or } \\
\text { fail }\end{array}$} & \multirow[b]{2}{*}{$\begin{array}{c}\text { Data } \\
\text { Records }\end{array}$} \\
\hline & $\begin{array}{c}\text { \# of } \\
\text { compacts }\end{array}$ & $\begin{array}{c}\text { \# of } \\
\text { particles }\end{array}$ & & & & & \\
\hline $\begin{array}{l}\text { Uranium contamination fraction } \\
\text { ( } g \text { exposed } U / g r a m ~ U \text { in compact) }\end{array}$ & 24 & 99470 & $\leq 1.0 \times 10^{-4}$ & $\leq 4$ effectively exposed kernels in 291533 particles & 0 & pass & $\begin{array}{l}\text { IRF-05C } \\
\text { DRF-26 }\end{array}$ \\
\hline $\begin{array}{l}\text { Defective SiC coating fraction } \\
\text { (fraction of total particles) }\end{array}$ & 12 & 49735 & $\leq 2.0 \times 10^{-4}$ & $\begin{array}{l}\leq 4 \text { leached kernels in } 245766 \text { particles } \\
\text { or } \leq 12 \text { leached kernels in } \geq 97210 \text { particles }\end{array}$ & 2 & pass & $\begin{array}{l}\text { IRF-05D } \\
\text { DRF-26 }\end{array}$ \\
\hline $\begin{array}{l}\text { Defective IPyC coating fraction } \\
\text { (fraction of total particles) }\end{array}$ & 12 & 49735 & $\leq 2.0 \times 10^{-4}$ & $\begin{array}{l}\leq 4 \text { with excessive } U \text { dispersion in } \geq 45766 \text { particles } \\
\text { or } \$ 12 \text { with excessive } U \text { dispersion in } \geq 97210 \text { particles }\end{array}$ & 0 & pass & DRF-28 \\
\hline $\begin{array}{l}\text { Defective OPyC coating fraction } \\
\text { (fraction of total particles) }\end{array}$ & 1 & 4145 & $\leq 0.01$ & $\begin{array}{l}\leq 6 \text { cracked or missing OPyC in } \geq 1182 \text { particles } \\
\text { or } \leq 30 \text { cracked or missing OPyC in } \geq 4064 \text { particles }\end{array}$ & 0 & pass & DRF-27 \\
\hline
\end{tabular}

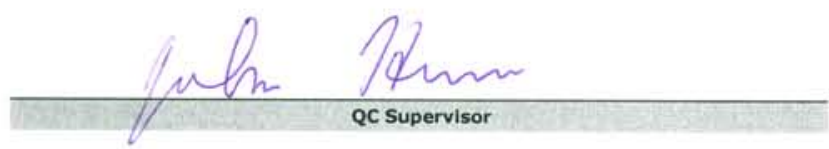

\begin{tabular}{|r|r|}
\hline Accept compact lot (Yes or No): & Yes \\
\hline
\end{tabular}

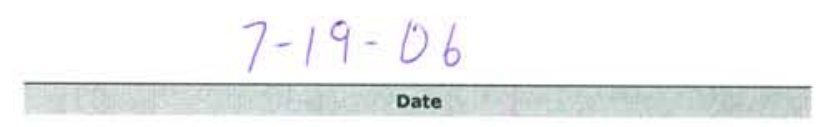

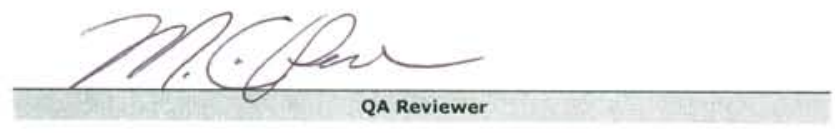

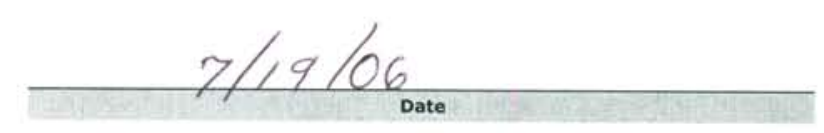


Inspection Report Form IRF-05B: Summary of Impurites Outside SiC

\begin{tabular}{|r|l|}
\hline Procedure: & AGR-CHAR-PIP-05 Rev. 0 \\
\hline Operator: & Fred Montgomery \\
\hline Compact lot ID: & LEU01-46T-Z \\
\hline Compact Lot description: & Compacts of AGR-1 baseline coated particle composite LEU01-46T \\
\hline
\end{tabular}

\begin{tabular}{|c|c|c|c|c|c|c|}
\hline Compact ID numbers: & $10,13,66$ & $40,48,64$ & $35,54,59$ & $08,26,75$ & Mean & $\begin{array}{l}\text { Standard } \\
\text { Deviation } \\
\end{array}$ \\
\hline Number of compacts: & 3 & 3 & 3 & 3 & & \\
\hline \multicolumn{7}{|l|}{$=\frac{1}{2}$} \\
\hline Deconsolidation-leach (DRF-26A) $(\mu \mathrm{g})$ : & 5.15 & 5.86 & 8.87 & 4.85 & & \\
\hline Burn-leach (DRF-26B) $(\mu \mathrm{g})$ : & 1.89 & 2.08 & 1.46 & 5.47 & & \\
\hline Total leached $(\mu \mathrm{g})$ : & 7.04 & 7.94 & 10.33 & 10.32 & & \\
\hline Fe outside SiC ( $\mu \mathrm{g} /$ compact): & 2.35 & 2.65 & 3.44 & 3.44 & 2.97 & 0.56 \\
\hline \multicolumn{7}{|l|}{ Chromium } \\
\hline Deconsolidation-leach (DRF-26A) $(\mu \mathrm{g})$ : & 3.36 & 3.38 & 3.36 & 3.22 & & \\
\hline Burn-leach (DRF-26B) $(\mu \mathrm{g})$; & 1.41 & 1.41 & 1.41 & 1.41 & & \\
\hline Total leached $(\mu \mathrm{g})$ : & 4.77 & 4.79 & 4.77 & 4.63 & & \\
\hline Cr outside SiC ( $\mu \mathrm{g} /$ compact): & 1.59 & 1.60 & 1.59 & 1.54 & 1.58 & 0.02 \\
\hline \multicolumn{7}{|l|}{ Manganese } \\
\hline Deconsolidation-leach (DRF-26A) $(\mu \mathrm{g})$ : & 0.85 & 0.86 & 1.19 & 1.11 & & \\
\hline Burn-leach (DRF-26B) $(\mu \mathrm{g})$ : & 0.20 & 0.20 & 0.37 & 0.20 & & \\
\hline Total leached $(\mu \mathrm{g})$ : & 1.05 & 1.07 & 1.56 & 1.32 & & \\
\hline Mn outside SiC ( $\mu \mathrm{g} /$ compact): & 0.35 & 0.36 & 0.52 & 0.44 & 0.42 & 0.08 \\
\hline \multicolumn{7}{|l|}{ Cobalt } \\
\hline Deconsolidation-leach (DRF-26A) $(\mu \mathrm{g})$ : & 1.34 & 1.35 & 1.34 & 1.28 & & \\
\hline Burn-leach (DRF-26B) $(\mu \mathrm{g})$ : & 0.56 & 0.56 & 0.56 & 0.56 & & \\
\hline Total leached $(\mu \mathrm{g})$ : & 1.90 & 1.92 & 1.90 & 1.85 & & \\
\hline Co outside SiC $(\mu \mathrm{g} /$ compact $)$ : & 0.63 & 0.64 & 0.63 & 0.62 & 0.63 & 0.01 \\
\hline \multicolumn{7}{|l|}{ Nickel } \\
\hline Deconsolidation-leach (DRF-26A) $(\mu \mathrm{g})$ : & 2.38 & 2.40 & 2.38 & 2.28 & & \\
\hline Burn-leach (DRF-26B) $(\mu g)$ : & 1.00 & 1.26 & 1.66 & 1.00 & & \\
\hline Total leached $(\mu \mathrm{g})$ : & 3.38 & 3.66 & 4.04 & 3.28 & & \\
\hline Ni outside SiC ( $\mu \mathrm{g} /$ compact): & 1.13 & 1.22 & 1.35 & 1.09 & 1.20 & 0.11 \\
\hline \multicolumn{7}{|l|}{ Transition Metals } \\
\hline $\mathrm{Cr}+\mathrm{Mn}+\mathrm{Co}+\mathrm{Ni}$ outside SiC $(\mu \mathrm{g} /$ compact $):$ & 3.70 & 3.81 & 4.09 & 3.69 & 3.82 & 0.19 \\
\hline \multicolumn{7}{|l|}{ Calcium } \\
\hline Deconsolidation-leach (DRF-26A) $(\mu \mathrm{g})$ : & 23.95 & 21.89 & 18.68 & 30.88 & & \\
\hline Burn-leach (DRF-26B) $(\mu g)$ : & 14.11 & 26.95 & 22.12 & 18.62 & & \\
\hline Total leached $(\mu g)$ : & 38.06 & 48.84 & 40.80 & 49.50 & & \\
\hline Ca outside SiC ( $\mu \mathrm{g} /$ compact): & 12.69 & 16.28 & 13.60 & 16.50 & 14.77 & 1.91 \\
\hline \multicolumn{7}{|l|}{ Aluminum } \\
\hline Deconsolidation-leach (DRF-26A) $(\mu \mathrm{g})$ : & 19.53 & 18.16 & 15.96 & 27.00 & & \\
\hline Burn-leach (DRF-26B) $(\mu \mathrm{g})$ : & 14.13 & 14.83 & 8.14 & 5.62 & & \\
\hline Total leached $(\mu \mathrm{g})$ : & 33.66 & 32.99 & 24.10 & 32.62 & & \\
\hline Al outside SiC ( $\mu \mathrm{g} /$ compact): & 11.22 & 11.00 & 8.03 & 10.87 & 10.28 & 1.51 \\
\hline \multicolumn{7}{|l|}{ Titanium } \\
\hline Deconsolidation-leach (DRF-26A) $(\mu \mathrm{g})$ : & 17.01 & 14.58 & 14.90 & 13.46 & & \\
\hline Burn-leach (DRF-26B) ( $\mu \mathrm{g})$ : & 4.96 & 12.76 & 14.09 & 16.26 & & \\
\hline Total leached $(\mu \mathrm{g})$ : & 21.97 & 27.34 & 28.99 & 29.72 & & \\
\hline Ti outside SiC ( $\mu \mathrm{g} /$ compact): & 7.32 & 9.11 & 9.66 & 9.91 & 9.00 & 1.17 \\
\hline \multicolumn{7}{|l|}{ Vanadium } \\
\hline Deconsolidation-leach (DRF-26A) $(\mu \mathrm{g})$ : & 29.60 & 28.43 & 30.98 & 30.26 & & \\
\hline Burn-leach (DRF-26B) $(\mu \mathrm{g})$ : & 22.51 & 21.42 & 27.54 & 27.60 & & \\
\hline Total leached $(\mu \mathrm{g})$ : & 52.11 & 49.85 & 58.52 & 57.86 & & \\
\hline V outside SiC ( $\mu \mathrm{g} /$ compact): & 17.37 & 16.62 & 19.51 & 19.29 & 18.20 & 1.42 \\
\hline \multicolumn{7}{|l|}{ Titanium and Vanadium } \\
\hline $\mathrm{Ti}+\mathrm{V}$ outside SiC ( $\mu \mathrm{g} /$ compact $):$ & 24.69 & 25.73 & 29.17 & 29.19 & 27.20 & 2.33 \\
\hline
\end{tabular}

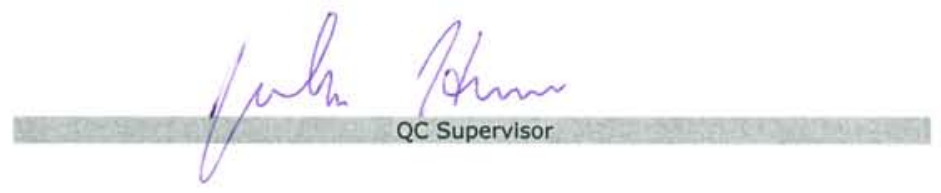

$$
5-11-06
$$


ORNL/TM-2006/507, Rev. 0

Inspection Report Form IRF-05C: Summary of Uranium Contamination

Procedure: AGR-CHAR-PIP-05 Rev. 0

Operator: Fred Montgomery

Compact lot ID: LEU01-46T-Z

Compact Lot description: Compacts of AGR-1 baseline coated particle composite LEU01-46T

\begin{tabular}{|r|c|c|c|c|c|c|}
\hline Compact ID numbers: & $10,13,66$ & $40,48,64$ & $35,54,59$ & $08,26,75$ & $\begin{array}{c}02,04,11,28, \\
31,61\end{array}$ & $\begin{array}{l}25,41,51,63, \\
70,73\end{array}$ \\
\hline \hline Number of compacts: & 3 & 3 & 3 & 3 & 6 & 6 \\
\hline Effective number of exposed kernels: & 0.0 & 0.0 & 0.0 & 0.0 & 0.0 & 0.0 \\
\hline
\end{tabular}

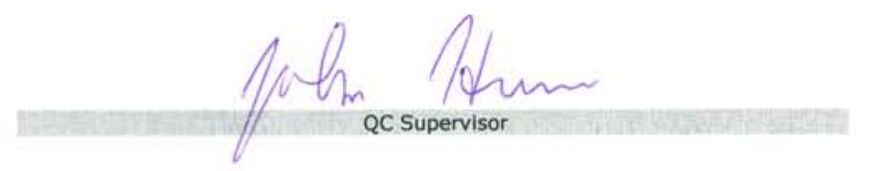

$$
5-11-06
$$


ORNL/TM-2006/507, Rev. 0

Inspection Report Form IRF-05D: Summary of SIC Burn-Leach Defects

Procedure: AGR-CHAR-PIP-05 ReV. 0

Operator: Fred Montgomery

Compact lot ID: LEU01-46T-Z

Compact Lot description: Compacts of AGR-1 baseline coated particle composite LEU01-46T

\begin{tabular}{|r|c|c|c|c|c|c|}
\hline Compact ID numbers: & $10,13,66$ & $40,48,64$ & $35,54,59$ & $08,26,75$ & Total \\
\hline \hline Number of compacts: & 3 & 3 & 3 & 3 & & \\
\hline Number of leached kernels: & 1 & 1 & 0 & 0 & 12 \\
\hline
\end{tabular}

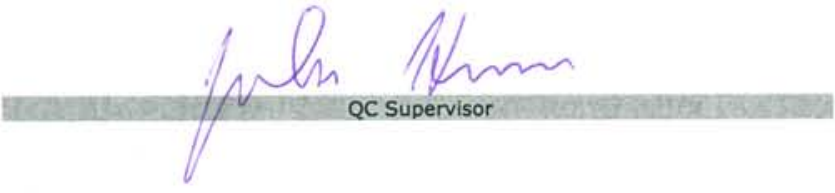

$$
5-11-06
$$




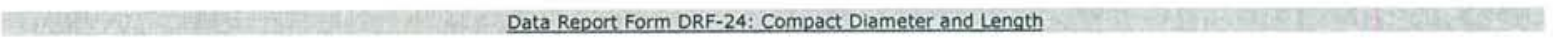

\begin{tabular}{rl|}
\hline Procedure: & AGR-CHAR-DAM-24 Rev. 3 \\
\hline Operator: & Ivan Dunbar \\
\hline Compact lot ID: & LEU01-46T-Z \\
\hline Compact Lot description: & Compacts of AGR-1 baseline coated particle composite LEU01-46T \\
\hline Filename: & IImC-agrVAGRICompactDimensions \LEU01-46T-Z_DRF24R3.xls \\
\hline \\
\begin{aligned} & \hline Vertical height gauge calibration due date: $9 / 8 / 06 \\
&$\hline Pass-thru block calibration due date: $1 / 17 / 09 \\
&$\hline Digital caliper calibration due date: $9 / 8 / 06 \\
&$\hline Gauge blocks calibration due date: $9 / 8 / 06 \\
&$\hline\end{aligned}
\end{tabular}

\begin{tabular}{|r|l|}
\hline Acceptance criteria for compact length: & $\geq 25.02$ and $\leq 25.40 \mathrm{~mm}$ \\
\hline Acceptance criteria for compact diameter: & $\geq 12.22$ and $\leq 12.46 \mathrm{~mm}$ (and pass through 12.46 mm ring gauge) \\
\hline Acceptance criteria for compact mass: & For information only \\
\hline
\end{tabular}

\begin{tabular}{|c|c|c|c|c|c|c|c|c|c|c|}
\hline \multirow{2}{*}{$\begin{array}{l}\text { Compact } \\
\text { ID Number }\end{array}$} & \multirow{2}{*}{$\begin{array}{l}\text { Length } \\
(\mathrm{mm})\end{array}$} & \multicolumn{6}{|c|}{ Diameter $(\mathrm{mm})$} & \multirow{2}{*}{$\begin{array}{l}\text { Pass Thru? } \\
(Y \text { or } \mathrm{N})\end{array}$} & \multirow{2}{*}{$\begin{array}{l}\text { Mass } \\
\text { (q) }\end{array}$} & \multirow{2}{*}{$\begin{array}{c}\text { Accept? } \\
\text { (pass or fail) }\end{array}$} \\
\hline & & Top 1 & Top 2 & Middle 1 & Middle 2 & Bottom 1 & Bottom 2 & & & \\
\hline 01 & 25.128 & 12.37 & 12.38 & 12.37 & 12.37 & 12.37 & 12.36 & $Y$ & 5.4794 & pass \\
\hline 02 & 25.030 & 12.37 & 12.36 & 12.37 & 12.36 & 12.36 & 12.36 & $Y$ & 5.4872 & pass \\
\hline 03 & 24.857 & 12.40 & 12.41 & 12.41 & 12.41 & 12.41 & 12.41 & $Y$ & 5.4757 & fail \\
\hline 04 & 25.090 & 12.37 & 12.37 & 12.37 & 12.37 & 12.36 & 12.36 & $Y$ & 5.4900 & pass \\
\hline 05 & 25.264 & 12.36 & 12.36 & 12.36 & 12.36 & 12.35 & 12.36 & $\bar{Y}$ & 5.4779 & pass \\
\hline 06 & 24.999 & 12.37 & 12.36 & 12.36 & 12.36 & 12.36 & 12.36 & $Y$ & 5.4878 & fail \\
\hline 07 & 25.006 & 12.37 & 12.37 & 12.37 & 12.37 & 12.36 & 12.36 & $Y$ & 5.4676 & fail \\
\hline 08 & 25.012 & 12.37 & 12.37 & 12.37 & 12.37 & 12.36 & 12.36 & $Y$ & 5.4863 & fail \\
\hline 09 & 25.013 & 12.37 & 12.37 & 12.37 & 12.37 & 12.35 & 12.36 & $Y$ & 5.4786 & fail \\
\hline 10 & 25.006 & 12.38 & 12.37 & 12.37 & 12.37 & 12.36 & 12.37 & $Y$ & 5.4874 & fail \\
\hline 11 & 25.100 & 12.37 & 12.37 & 12.38 & 12.37 & 12.36 & 12.37 & $Y$ & 5.4893 & pass \\
\hline 12 & 24.985 & 12.37 & 12.37 & 12.37 & 12.37 & 12.36 & 12.36 & $Y$ & 5.4890 & fail \\
\hline 13 & 25.039 & 12.38 & 12.38 & 12.38 & 12.37 & 12.36 & 12.36 & $Y$ & 5.4669 & pass \\
\hline 14 & 24.997 & 12.37 & 12.37 & 12.37 & 12.36 & 12.36 & 12.36 & $\bar{Y}$ & 5.4797 & fail \\
\hline 15 & 25.030 & 12.37 & 12.37 & 12.37 & 12.37 & 12.36 & 12.36 & $Y$ & 5.4835 & pass \\
\hline 16 & 25.016 & 12.36 & 12.36 & 12.37 & 12.36 & 12.35 & 12.35 & $Y$ & 5.4879 & fail \\
\hline 17 & 25.074 & 12.37 & 12.37 & 12.37 & 12.37 & 12.36 & 12.36 & $Y$ & 5.4861 & pass \\
\hline 18 & 24.960 & 12.37 & 12.37 & 12.38 & 12.37 & 12.36 & 12.36 & $Y$ & 5.4730 & fail \\
\hline 19 & 25.256 & 12.36 & 12.37 & 12.36 & 12.36 & 12.35 & 12.35 & $Y$ & 5.4874 & pass \\
\hline 20 & 25.003 & 12.38 & 12.38 & 12.38 & 12.38 & 12.37 & 12.37 & $Y$ & 5.4855 & fail \\
\hline 21 & 25.017 & 12.37 & 12.37 & 12.37 & 12.37 & 12.36 & 12.36 & $Y$ & 5.4967 & fail \\
\hline 22 & 25.053 & 12.38 & 12.37 & 12.37 & 12.38 & 12.36 & 12.37 & $Y$ & 5.4980 & pass \\
\hline 23 & 24.994 & 12.37 & 12.37 & 12.37 & 12.37 & 12.36 & 12.36 & $Y$ & 5.4814 & fail \\
\hline 24 & 25.022 & 12.37 & 12.37 & 12.37 & 12.37 & 12.36 & 12.36 & $Y$ & 5.4810 & pass \\
\hline 25 & 25.012 & 12.36 & 12.37 & 12.36 & 12.37 & 12.35 & 12.35 & $Y$ & 5.4664 & fail \\
\hline 26 & 25.008 & 12.38 & 12.38 & 12.37 & 12.37 & 12.36 & 12.36 & $Y$ & 5.4725 & fail \\
\hline 27 & 24.992 & 12.38 & 12.38 & 12.38 & 12.38 & 12.36 & 12.36 & $Y$ & 5.4936 & fail \\
\hline 28 & 25.125 & 12.37 & 12.36 & 12.37 & 12.37 & 12.36 & 12.36 & $Y$ & 5.4878 & pass \\
\hline 29 & 25.114 & 12.38 & 12.38 & 12.38 & 12.38 & 12.37 & 12.36 & $Y$ & 5.4826 & pass \\
\hline 30 & 25.115 & 12.37 & 12.36 & 12.37 & 12.37 & 12.35 & 12.36 & $Y$ & 5.4756 & pass \\
\hline 31 & 25.026 & 12.37 & 12.37 & 12.38 & 12.37 & 12.37 & 12.36 & $Y$ & 5.4800 & pass \\
\hline 32 & 25.005 & 12.37 & 12.37 & 12.37 & 12.37 & 12.37 & 12.36 & $Y$ & 5.4732 & fail \\
\hline 33 & 25.109 & 12.37 & 12.37 & 12.37 & 12.37 & 12.37 & 12.36 & $Y$ & 5.5023 & pass \\
\hline 34 & 25.097 & 12.37 & 12.36 & 12.37 & 12.36 & 12.36 & 12.36 & $Y$ & 5.4893 & pass \\
\hline 35 & 25.044 & 12.37 & 12.36 & 12.37 & 12.37 & 12.36 & 12.36 & $Y$ & 5.4941 & pass \\
\hline 36 & 25.031 & 12.37 & 12.37 & 12.37 & 12.37 & 12.36 & 12.36 & $Y$ & 5.4655 & pass \\
\hline 37 & 24.938 & 12.38 & 12.37 & 12.37 & 12.37 & 12.36 & 12.36 & $Y$ & 5.4757 & fail \\
\hline 38 & 24.941 & 12.37 & 12.37 & 12.38 & 12.38 & 12.37 & 12.38 & $Y$ & 5.4797 & fail \\
\hline 39 & 25.147 & 12.37 & 12.37 & 12.37 & 12.37 & 12.36 & 12.36 & $Y$ & 5.5035 & pass \\
\hline 40 & 25.073 & 12.37 & 12.37 & 12.38 & 12.38 & 12.37 & 12.36 & $Y$ & 5.4913 & pass \\
\hline
\end{tabular}

For diameter measurement, top corresponds to compact end at top during pressing.

Top end cap appears shinier than bottom.

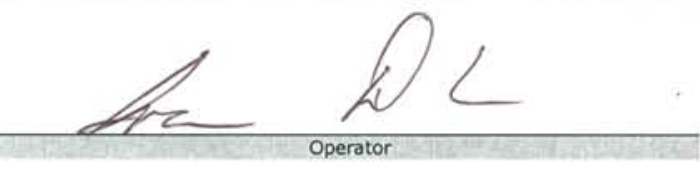

$$
\text { J-9-06 }
$$

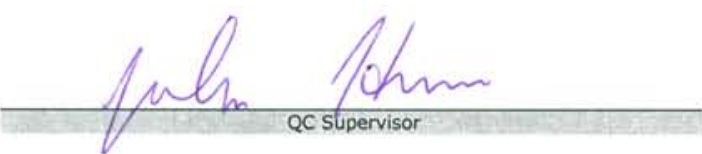

$$
5-3-06
$$
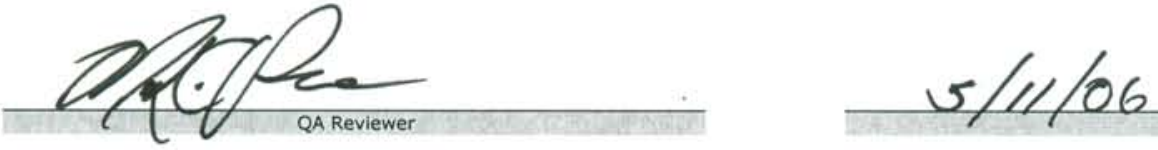
Wata Report Form DRF-24: Compact Diameter and Length

\begin{tabular}{|c|c|}
\hline Procedure: & AGR-CHAR-DAM-24 Rev. 3 \\
\hline Operator: & Ivan Dunbar \\
\hline Compact lot ID: & LEU01-46T-Z \\
\hline Compact Lot description: & Compacts of AGR-1 baseline coated particle composite LEU01-46T \\
\hline Filename: & IImc-agr|AGRICompactDimensions|LEU01-46T-Z_DRF24R3.xIs \\
\hline Vertical height gauge calibration due date: & $9 / 8 / 06$ \\
\hline Pass-thru block calibration due date: & $1 / 17 / 09$ \\
\hline Digital caliper calibration due date: & $9 / 8 / 06$ \\
\hline Gauge blocks calibration due date: & $9 / 8 / 06$ \\
\hline
\end{tabular}

\begin{tabular}{|r|l|}
\hline Acceptance criteria for compact length: & $\geq 25.02$ and $\leq 25.40 \mathrm{~mm}$ \\
\hline Acceptance criteria for compact diameter: & $\geq 12.22$ and $\leq 12.46 \mathrm{~mm}$ (and pass through $12.46 \mathrm{~mm}$ ring gauge) \\
\hline Acceptance criteria for compact mass: & For information only \\
\hline
\end{tabular}

\begin{tabular}{|c|c|c|c|c|c|c|c|c|c|c|}
\hline \multirow{2}{*}{$\begin{array}{l}\text { Compact } \\
\text { ID Number }\end{array}$} & \multirow{2}{*}{$\begin{array}{l}\text { Length } \\
(\mathrm{mm})\end{array}$} & \multicolumn{6}{|c|}{ Diameter $(\mathrm{mm})$} & \multirow{2}{*}{$\begin{array}{l}\text { Pass Thru? } \\
(Y \circ \text { or })\end{array}$} & \multirow{2}{*}{$\begin{array}{l}\text { Mass } \\
\text { (a) }\end{array}$} & \multirow{2}{*}{$\begin{array}{c}\text { Accept? } \\
\text { (pass or fail) }\end{array}$} \\
\hline & & Top 1 & Top 2 & Middle 1 & Middle 2 & Bottom 1 & Bottom 2 & & & \\
\hline 41 & 25.052 & 12.38 & 12.38 & 12.38 & 12.37 & 12.36 & 12.36 & $Y$ & 5.4934 & pass \\
\hline 42 & 25.059 & 12.37 & 12.37 & 12.36 & 12.36 & 12.35 & 12.36 & $Y$ & 5.4867 & pass \\
\hline 43 & 25.133 & 12.37 & 12.36 & 12.36 & 12.37 & 12.36 & 12.36 & $Y$ & 5.4941 & pass \\
\hline 44 & 25.027 & 12.37 & 12.37 & 12.36 & 12.37 & 12.37 & 12.36 & $Y$ & 5.4851 & pass \\
\hline 45 & 25.077 & 12.36 & 12.36 & 12.36 & 12.36 & 12.36 & 12.36 & $Y$ & 5.4670 & pass \\
\hline 46 & 25.074 & 12.36 & 12.36 & 12.36 & 12.36 & 12.35 & 12.35 & $Y$ & 5.4631 & pass \\
\hline 47 & 25.041 & 12.37 & 12.37 & 12.37 & 12.37 & 12.37 & 12.37 & $Y$ & 5.4802 & pass \\
\hline 48 & 25.046 & 12.37 & 12.37 & 12.37 & 12.37 & 12.36 & 12.37 & $Y$ & 5.4875 & pass \\
\hline 49 & 25.090 & 12.37 & 12.37 & 12.37 & 12.37 & 12.37 & 12.36 & $Y$ & 5.4791 & pass \\
\hline 50 & 25.140 & 12.37 & 12.37 & 12.37 & 12.37 & 12.36 & 12.35 & $Y$ & 5.4843 & pass \\
\hline 51 & 25.086 & 12.36 & 12.36 & 12.37 & 12.36 & 12.36 & 12.35 & $Y$ & 5.4649 & pass \\
\hline 52 & 25.143 & 12.36 & 12.36 & 12.36 & 12.36 & 12.36 & 12.35 & $Y$ & 5.4792 & pass \\
\hline 53 & 25.022 & 12.37 & 12.37 & 12.37 & 12.37 & 12.37 & 12.36 & $Y$ & 5.4877 & pass \\
\hline 54 & 25.046 & 12.37 & 12.37 & 12.37 & 12.36 & 12.36 & 12.35 & $Y$ & 5.4938 & pass \\
\hline 55 & 25.065 & 12.37 & 12.37 & 12.37 & 12.37 & 12.36 & 12.36 & $Y$ & 5.4907 & pass \\
\hline 56 & 25.073 & 12.37 & 12.36 & 12.37 & 12.37 & 12.36 & 12.37 & $Y$ & 5.4913 & pass \\
\hline 57 & 25.142 & 12.36 & 12.36 & 12.37 & 12.37 & 12.35 & 12.35 & $Y$ & 5.4829 & pass \\
\hline 58 & 25.073 & 12.36 & 12.36 & 12.36 & 12.36 & 12.35 & 12.35 & $Y$ & 5.4760 & pass \\
\hline 59 & 25.055 & 12.36 & 12.36 & 12.37 & 12.36 & 12.36 & 12.35 & $Y$ & 5.4883 & pass \\
\hline 60 & 25.250 & 12.36 & 12.36 & 12.36 & 12.36 & 12.36 & 12.35 & $Y$ & 5.4757 & pass \\
\hline 61 & 25.015 & 12.37 & 12.37 & 12.37 & 12.37 & 12.36 & 12.36 & $Y$ & 5.4877 & fail \\
\hline 62 & 25.135 & 12.36 & 12.36 & 12.36 & 12.36 & 12.35 & 12.35 & $Y$ & 5.4719 & pass \\
\hline 63 & 24.997 & 12.37 & 12.37 & 12.37 & 12.37 & 12.36 & 12.36 & $Y$ & 5.4895 & fail \\
\hline 64 & 25.045 & 12.37 & 12.37 & 12.37 & 12.37 & 12.36 & 12.36 & $Y$ & 5.5164 & pass \\
\hline 65 & 25.032 & 12.37 & 12.37 & 12.37 & 12.37 & 12.36 & 12.36 & $Y$ & 5.4856 & pass \\
\hline 66 & 24.977 & 12.36 & 12.37 & 12.36 & 12.37 & 12.36 & 12.36 & $Y$ & 5.4737 & fail \\
\hline 67 & 25.001 & 12.36 & 12.36 & 12.36 & 12.36 & 12.35 & 12.36 & $Y$ & 5.4546 & fail \\
\hline 68 & 24.928 & 12.37 & 12.36 & 12.37 & 12.37 & 12.36 & 12.36 & $Y$ & 5.4756 & fail \\
\hline 69 & 25.036 & 12.37 & 12.37 & 12.37 & 12.37 & 12.35 & 12.36 & $Y$ & 5.4695 & pass \\
\hline 70 & 25.024 & 12.37 & 12.37 & 12.37 & 12.37 & 12.36 & 12.36 & $Y$ & 5.4930 & pass \\
\hline 71 & 25.041 & 12.36 & 12.37 & 12.36 & 12.37 & 12.35 & 12.35 & $Y$ & 5.5056 & pass \\
\hline 72 & 25.012 & 12.37 & 12.36 & 12.36 & 12.37 & 12.36 & 12.36 & $Y$ & 5.4745 & fail \\
\hline 73 & 25.262 & 12.36 & 12.36 & 12.36 & 12.37 & 12.35 & 12.36 & $Y$ & 5.4795 & pass \\
\hline 74 & 24.996 & 12.37 & 12.37 & 12.38 & 12.38 & 12.36 & 12.36 & $Y$ & 5.4793 & fail \\
\hline 75 & 25.030 & 12.36 & 12.37 & 12.37 & 12.37 & 12.36 & 12.36 & $Y$ & 5.4941 & pass \\
\hline 76 & 24.942 & 12.37 & 12.37 & 12.37 & 12.37 & 12.36 & 12.37 & $Y$ & 5.4706 & fail \\
\hline 77 & 24.997 & 12.36 & 12.37 & 12.37 & 12.37 & 12.36 & 12.36 & $Y$ & 5.4648 & fail \\
\hline 78 & 24.960 & 12.36 & 12.36 & 12.36 & 12.37 & 12.36 & 12.36 & $Y$ & 5.4374 & fail \\
\hline 79 & 24.985 & 12.37 & 12.37 & 12.37 & 12.37 & 12.36 & 12.36 & $Y$ & 5.4721 & fail \\
\hline
\end{tabular}

For diameter measurement, top corresponds to compact end at top during pressing.

Top end cap appears shinier than bottom.

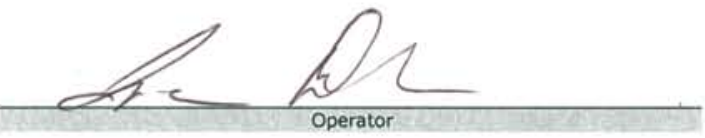

$$
3-9-66
$$

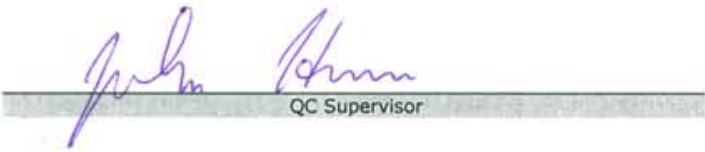

$$
5-3-06
$$
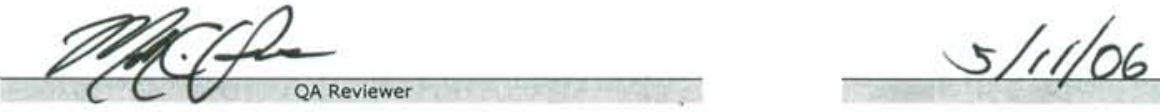
ORNL/TM-2006/507, Rev. 0

Data Report Form DRF-25: Fuel Compact Mean Uranium Loading

\begin{tabular}{|r|l|}
\hline Procedure: & AGR-CHAR-DAM-25 Rev. 1 \\
\hline Operator: & Montgomery \\
\hline Compact lot ID: & LEU01-46T-Z \\
\hline Compact lot description: & Compacts of AGR-1 baselilne coated particle composite LEU01-46T \\
\hline Filename: & IImc-agr\AGR\UraniumLoading $\backslash$ LEU01-46T-Z_DRF25R1.xIs \\
\hline
\end{tabular}

\begin{tabular}{|c|c|c|c|c|c|c|}
\hline & Sample 1 & Sample 2 & Sample 3 & Sample 4 & Sample 5 & Sample 6 \\
\hline Compact ID number: & 1 & 16 & 21 & 45 & 71 & 72 \\
\hline Sample tube ID number: & U06031301 & U06031302 & U06031303 & U06031304 & U06031305 & U06031306 \\
\hline Radiochemical laboratory analysis number: & $060315-031$ & $060315-032$ & $060315-033$ & $060315-034$ & $060315-035$ & $060315-036$ \\
\hline Measured $U$ in compact $(g):$ & 0.92164 & 0.92012 & 0.92043 & 0.90734 & 0.91301 & 0.91697 \\
\hline Uncertainty in measured $U$ in compact $(\mathrm{g})$ : & 0.00092 & 0.00092 & 0.00092 & 0.00091 & 0.00091 & 0.00092 \\
\hline
\end{tabular}

\begin{tabular}{r|r} 
Mean uranium loading $(\mathrm{gU} /$ compact $):$ & 0.917 \\
\hline Standard deviation in mean uranium loading $(\mathrm{gU} /$ compact $):$ & 0.005
\end{tabular}

\section{Comments}

Four additional Davies-Gray analyses were performed on sample 1, grams U in compact: $0.92057,0.92209,0.92177,0.92167$.

Mean of five measurements on sample 1: $0.9215 \mathrm{~g}$, Standard error in mean: $0.0003 \mathrm{~g}$.

Data checked against official analysis results $5 / 3 / 06$.

Feed c. Montgomey
$5 / 3 / 06$ Date 
Data Report Form DRF-26A: Measurement of U Contamination and Impurities by Deconsolidation Leach

\begin{tabular}{|r|l|}
\hline Procedure: & AGR-CHAR-DAM-26 Rev. 0 \\
\hline Operator: & Fred Montgomery \\
\hline Compact lot ID: & LEU01-46T-Z \\
\hline Compact lot description: & Compacts of AGR-1 baseline coated particle composite LEU01-46T \\
\hline Compact ID numbers: & $10,13,66$ \\
\hline DRF filename: & IImc-agrVAGR LeachBurnLeach ILEU01-46T-Z_DRF26R0.xIs \\
\hline
\end{tabular}

\begin{tabular}{|r|c|}
\hline Mean average weight/kernel $(\mathrm{g}):$ & $2.42 \mathrm{E}-04$ \\
\hline Uncertainty in mean average weight/kernel $(\mathrm{g}):$ & $5.96 \mathrm{E}-07$ \\
\hline Mean weight \% uranium/kernel: & 90.06 \\
\hline Standard deviation in weight \% uranium/kernel: & 0.09 \\
\hline Approximate weight uranium/kernel $(\mathrm{g}):$ & $2.18 \mathrm{E}-04$ \\
\hline Uncertainty in approx. weight uranium/kernel $(\mathrm{g}):$ & $5.76 \mathrm{E}-07$ \\
\hline
\end{tabular}

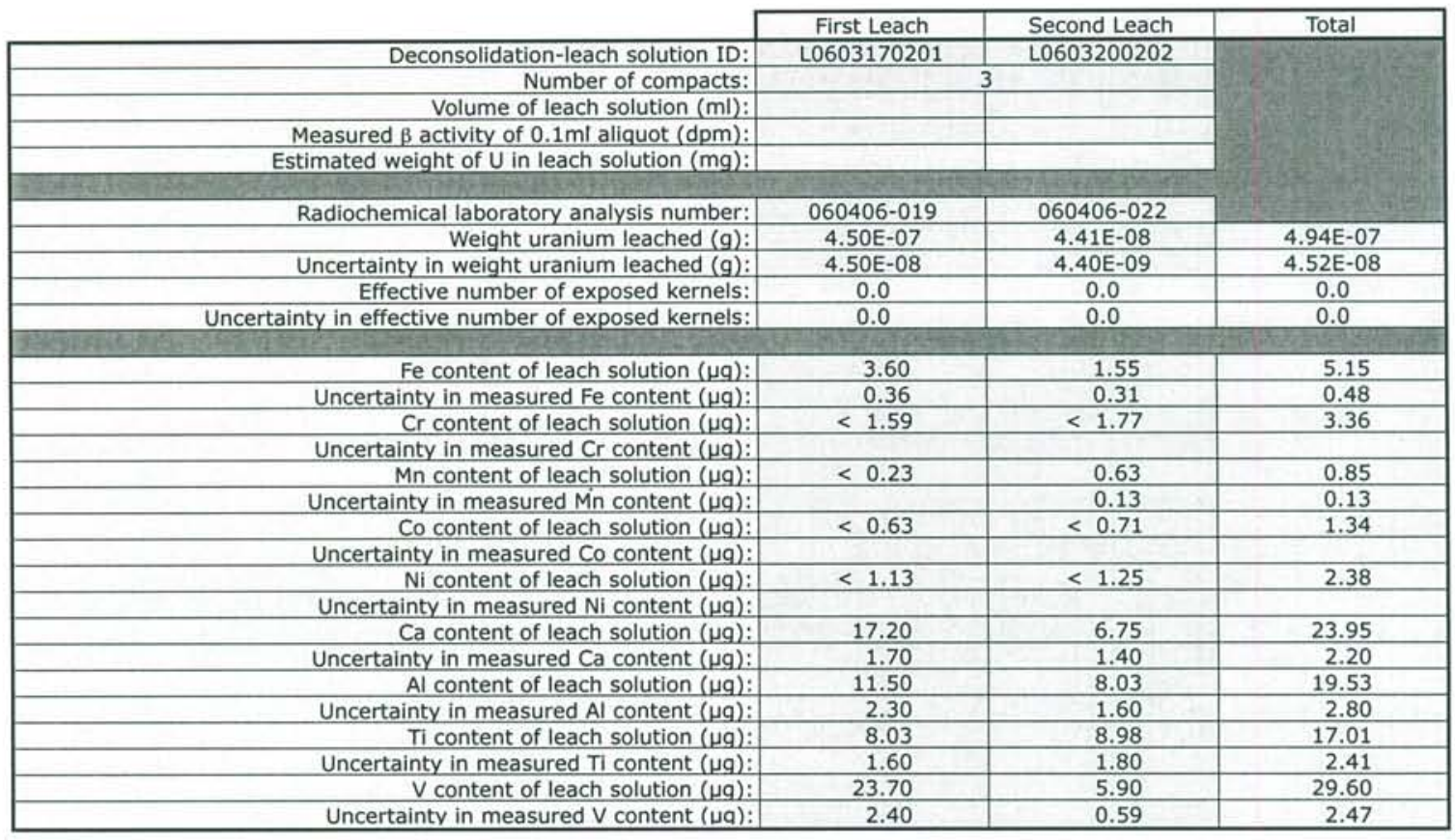

Comments

\section{Foed c. Montgomery}

$5 / 2 / 06$

Date

$$
\text { 更 }
$$


Data Report Form DRF-26A: Measurement of U Contamination and Impurities by Deconsolidation Leach

\begin{tabular}{|r|l|}
\hline Procedure: & AGR-CHAR-DAM-26 Rev. 0 \\
\hline Operator: & Fred Montgomery \\
\hline Compact lot ID: & LEU01-46T-Z \\
\hline Compact lot description: & Compacts of AGR-1 baseline coated particle composite LEU01-46T \\
\hline Compact ID numbers: & $40,48,64$ \\
\hline DRF filename: & \Imc-agr\AGR\LeachBurnLeach \LEU01-46T-Z_DRF26R0.xIs \\
\hline
\end{tabular}

\begin{tabular}{|r|c|}
\hline Mean average weight/kernel $(\mathrm{g}):$ & $2.42 \mathrm{E}-04$ \\
\hline Uncertainty in mean average weight/kernel $(\mathrm{g}):$ & $5.96 \mathrm{E}-07$ \\
\hline Mean weight \% uranium/kernel: & 90.06 \\
\hline Standard deviation in weight \% uranium/kernel: & 0.09 \\
\hline Approximate weight uranium/kernel $(\mathrm{g}):$ & $2.18 \mathrm{E}-04$ \\
\hline Uncertainty in approx. weight uranium/kernel $(\mathrm{g}):$ & $5.76 \mathrm{E}-07$ \\
\hline
\end{tabular}

\begin{tabular}{|c|c|c|c|}
\hline & First Leach & Second Leach & Total \\
\hline Deconsolidation-leach solution ID: & L0603170301 & L0603200302 & \\
\hline Number of compacts: & \multicolumn{2}{|l|}{ 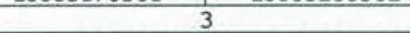 } & \\
\hline \multicolumn{4}{|l|}{ Volume of leach solution $(\mathrm{ml})$ : } \\
\hline \multicolumn{4}{|l|}{ Measured $\beta$ activity of $0.1 \mathrm{ml}$ aliquot (dpm): } \\
\hline \multirow{2}{*}{\multicolumn{4}{|c|}{ Estimated weight of $U$ in leach solution $(\mathrm{mg})$ : }} \\
\hline 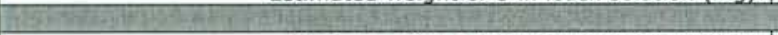 & & & \\
\hline \multicolumn{4}{|l|}{ Radiochemical laboratory analysis number: } \\
\hline Weight uranium leached $(g)$ : & $7.00 E-07$ & $9.46 \mathrm{E}-08$ & $7.95 E-07$ \\
\hline Uncertainty in weight uranium leached $(g)$ : & $7.00 \mathrm{E}-08$ & $9.50 \mathrm{E}-09$ & $7.06 \mathrm{E}-08$ \\
\hline Effective number of exposed kernels: & 0.0 & 0.0 & 0.0 \\
\hline Uncertainty in effective number of exposed kernels: & 0.0 & 0.0 & 0.0 \\
\hline \multicolumn{4}{|l|}{ 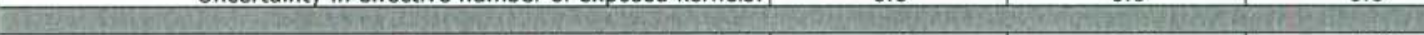 } \\
\hline Fe content of leach solution $(\mu \mathrm{g})$ : & 4.41 & 1.45 & 5.86 \\
\hline Uncertainty in measured Fe content $(\mu \mathrm{g})$ : & 0.44 & 0.29 & 0.53 \\
\hline $\mathrm{Cr}$ content of leach solution $(\mu \mathrm{g})$ : & $<1.58$ & $<1.80$ & 3.38 \\
\hline \multicolumn{4}{|l|}{ Uncertainty in measured $\mathrm{Cr}$ content $(\mu \mathrm{g})$ : } \\
\hline Mn content of leach solution $(\mu \mathrm{g})$ : & $<0.23$ & 0.64 & 0.86 \\
\hline Uncertainty in measured $\mathrm{Mn}$ content $(\mu \mathrm{q})$ : & & 0.13 & 0.13 \\
\hline Co content of leach solution $(\mu \mathrm{g})$ : & $<0.63$ & $<0.72$ & 1.35 \\
\hline \multicolumn{4}{|l|}{ Uncertainty in measured Co content $(\mu \mathrm{g})$ : } \\
\hline Ni content of leach solution $(\mu \mathrm{g})$ : & $<1.12$ & $<1.28$ & 2.40 \\
\hline \multicolumn{4}{|l|}{ Uncertainty in measured Ni content $(\mu \mathrm{g})$ : } \\
\hline Ca content of leach solution $(\mu \mathrm{g})$ : & 14.90 & 6.99 & 21.89 \\
\hline Uncertainty in measured Ca content $(\mu \mathrm{g})$ : & 1.50 & 1.40 & 2.05 \\
\hline Al content of leach solution $(\mu \mathrm{g})$ : & 9.90 & 8.26 & 18.16 \\
\hline Uncertainty in measured $\mathrm{Al}$ content $(\mu \mathrm{q})$ : & 2.00 & 1.70 & 2.62 \\
\hline $\mathrm{Ti}$ content of leach solution $(\mu \mathrm{g})$ : & 6.32 & 8.26 & 14.58 \\
\hline Uncertainty in measured $\mathrm{Ti}$ content $(\mu \mathrm{g})$ : & 1.30 & 1.70 & 2.14 \\
\hline$\checkmark$ content of leach solution $(\mu \mathrm{g})$ & 23.30 & 5.13 & 28.43 \\
\hline Uncertainty in measured $\mathrm{V}$ content $(\mathrm{uq})$ : & 2.30 & 0.51 & 2.36 \\
\hline
\end{tabular}

Comments

\section{Fued c. Montgomery}

\section{$5 / 2 / 06$}


Data Report Form DRF-26A: Measurement of U Contamination and Impurities by Deconsolidation Leach

\begin{tabular}{|r|l|}
\hline Procedure: & AGR-CHAR-DAM-26 Rev. 0 \\
\hline Operator: & Fred Montgomery \\
\hline Compact lot ID: & LEU01-46T-Z \\
\hline Compact lot description: & Compacts of AGR-1 baseline coated particle composite LEU01-46T \\
\hline Compact ID numbers: & $35,54,59$ \\
\hline DRF filename: & $\backslash$ IImc-agr\AGR\LeachBurnLeach \LEU01-46T-Z_DRF26R0.xIs \\
\hline
\end{tabular}

\begin{tabular}{|r|c|}
\hline Mean average weight/kernel $(\mathrm{g}):$ & $2.42 \mathrm{E}-04$ \\
\hline Uncertainty in mean average weight/kernel $(\mathrm{g}):$ & $5.96 \mathrm{E}-07$ \\
\hline Mean weight \% uranium/kernel: & 90.06 \\
\hline Standard deviation in weight \% uranium/kernel: & 0.09 \\
\hline Approximate weight uranium/kernel $(\mathrm{g}):$ & $2.18 \mathrm{E}-04$ \\
\hline Uncertainty in approx. weight uranium/kernel $(\mathrm{g}):$ & $5.76 \mathrm{E}-07$ \\
\hline
\end{tabular}

\begin{tabular}{|c|c|c|c|}
\hline & First Leach & Second Leach & Total \\
\hline Deconsolidation-leach solution ID: & $L 0603210101$ & L0603230102 & \\
\hline Number of compacts: & \multicolumn{2}{|c|}{3} & \\
\hline \multicolumn{4}{|l|}{ Volume of leach solution $(\mathrm{ml})$ : } \\
\hline \multirow{2}{*}{\multicolumn{4}{|c|}{$\begin{array}{r}\text { Measured } \beta \text { activity of } 0.1 \mathrm{ml} \text { aliquot }(\mathrm{dpm}) \text { : } \\
\text { Estimated weight of } U \text { in leach solution }(\mathrm{mg}) \text { : }\end{array}$}} \\
\hline & & & \\
\hline \multicolumn{4}{|l|}{ Radiochemical laboratory analysis number: } \\
\hline Weight uranium leached $(g)$ : & $1.07 \mathrm{E}-08$ & $7.66 \mathrm{E}-08$ & 8.73E-08 \\
\hline Uncertainty in weight uranium leached $(\mathrm{g})$ : & $1.10 \mathrm{E}-09$ & 7.70E-09 & $7.78 \mathrm{E}-09$ \\
\hline Effective number of exposed kernels: & 0.0 & 0.0 & 0.0 \\
\hline Uncertainty in effective number of exposed kernels: & 0.0 & 0.0 & 0.0 \\
\hline \multicolumn{4}{|l|}{ 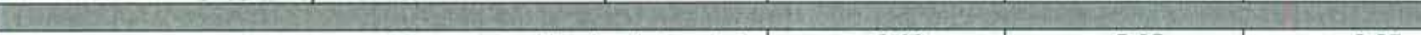 } \\
\hline Fe content of leach solution $(\mu \mathrm{g})$ : & 3.02 & 5.85 & 8.87 \\
\hline Uncertainty in measured $\mathrm{Fe}$ content $(\mu \mathrm{q})$ : & 0.30 & 0.59 & 0.66 \\
\hline $\mathrm{Cr}$ content of leach solution $(\mu \mathrm{g})$ : & $<1.59$ & $<1.77$ & 3.36 \\
\hline \multicolumn{4}{|l|}{ Uncertainty in measured $\mathrm{Cr}$ content $(\mu \mathrm{q})$ : } \\
\hline Mn content of leach solution $(\mu \mathrm{g})$ : & 0.59 & 0.60 & 1.19 \\
\hline Uncertainty in measured $\mathrm{Mn}$ content $(\mu \mathrm{g})$ : & 0.12 & 0.12 & 0.17 \\
\hline Co content of leach solution $(\mu \mathrm{g})$ : & $<0.63$ & $<0.71$ & 1.34 \\
\hline \multicolumn{4}{|l|}{ Uncertainty in measured Co content $(\mu \mathrm{g})$ : } \\
\hline Ni content of leach solution $(\mu \mathrm{g})$ : & $<1.13$ & $<1.25$ & 2.38 \\
\hline \multicolumn{4}{|l|}{ Uncertainty in measured $\mathrm{Ni}$ content $(\mu \mathrm{g})$ : } \\
\hline Ca content of leach solution $(\mu \mathrm{g})$ : & 15.20 & 3.48 & 18.68 \\
\hline Uncertainty in measured $\mathrm{Ca}$ content $(\mu \mathrm{q})$ : & 1.50 & 0.70 & 1.66 \\
\hline Al content of leach solution $(\mu \mathrm{g})$ : & 9.14 & $<6.82$ & 15.96 \\
\hline Uncertainty in measured $\mathrm{Al}$ content $(\mu \mathrm{g})$ : & 1.80 & & 1.80 \\
\hline TI content of leach solution $(\mu \mathrm{g})$ : & 6.55 & 8.35 & 14.90 \\
\hline Uncertainty in measured $\mathrm{Ti}$ content $(\mu \mathrm{g})$ : & 1.30 & 1.70 & 2.14 \\
\hline$V$ content of leach solution $(\mu \mathrm{g})$ : & 24.40 & 6.58 & 30.98 \\
\hline Uncertainty in measured $\mathrm{V}$ content ( $\mu \mathrm{a})$ : & 2.40 & 0.66 & 2.49 \\
\hline
\end{tabular}

Comments

\begin{tabular}{|l|l|}
\hline Data checked against official analysis results IPA14213 on $5 / 2 / 06$ \\
\hline
\end{tabular}

\section{Fued c. montgomeny}

$$
5 / 2 / 06
$$


Data Report Form DRF-26A: Measurement of U Contamination and Impurities by Deconsolidation Leach

\begin{tabular}{|c|c|}
\hline Procedure: & AGR-CHAR-DAM-26 Rev. 0 \\
\hline Operator: & Fred Montgomery \\
\hline Compact lot ID: & LEU $01-46 T-Z$ \\
\hline Compact lot description: & Compacts of AGR-1 baseline coated particle composite LEU01-46T \\
\hline Compact ID numbers: & $08,26,75$ \\
\hline DRF filename: & 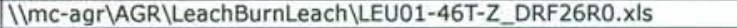 \\
\hline
\end{tabular}

\begin{tabular}{|r|c|}
\hline Mean average weight/kernel $(g):$ & $2.42 \mathrm{E}-04$ \\
\hline Uncertainty in mean average weight/kernel $(\mathrm{g}):$ & $5.96 \mathrm{E}-07$ \\
\hline Mean weight \% uranium/kernel: & 90.06 \\
\hline Standard deviation in weight \% uranium/kernel: & 0.09 \\
\hline Approximate weight uranium/kernel $(\mathrm{g}):$ & $2.18 \mathrm{E}-04$ \\
\hline Uncertainty in approx. weight uranium/kernel $(\mathrm{g}):$ & $5.76 \mathrm{E}-07$ \\
\hline
\end{tabular}

\begin{tabular}{|c|c|c|c|}
\hline & First Leach & Second Leach & Total \\
\hline Deconsolidation-leach solution ID: & L0603210201 & L0603230202 & \\
\hline Number of compacts: & \multicolumn{2}{|c|}{3} & \\
\hline \multicolumn{4}{|l|}{ Volume of leach solution $(\mathrm{ml})$ : } \\
\hline \multirow{2}{*}{\multicolumn{4}{|c|}{$\begin{array}{l}\text { Measured } \beta \text { activity of } 0.1 \mathrm{ml} \text { aliquot }(\mathrm{dpm}) \\
\text { Estimated weight of } U \text { in leach solution }(\mathrm{mg}) \text { : }\end{array}$}} \\
\hline & & & \\
\hline \multicolumn{4}{|l|}{ Radiochemical laboratory analysis number: } \\
\hline Weight uranium leached $(g)$ : & $6.36 \mathrm{E}-07$ & $5.22 \mathrm{E}-08$ & $6.88 E-07$ \\
\hline Uncertainty in weight uranium leached $(\mathrm{g})$ : & $6.40 \mathrm{E}-08$ & $5.20 \mathrm{E}-09$ & $6.42 E-08$ \\
\hline Effective number of exposed kernels: & 0.0 & 0.0 & 0.0 \\
\hline Uncertainty in effective number of exposed kernels: & 0.0 & 0.0 & 0.0 \\
\hline & & & \\
\hline Fe content of leach solution $(\mu \mathrm{g})$ : & 2.67 & 2.18 & 4.85 \\
\hline Uncertainty in measured Fe content $(\mu q)$ : & 0.53 & 0.44 & 0.69 \\
\hline $\mathrm{Cr}$ content of leach solution $(\mu \mathrm{g})$ : & $<1.52$ & $<1.70$ & 3.22 \\
\hline \multicolumn{4}{|l|}{ Uncertainty in measured $\mathrm{Cr}$ content $(\mu \mathrm{g})$ : } \\
\hline Mn content of leach solution $(\mu \mathrm{g})$ : & 0.54 & 0.58 & 1.11 \\
\hline Uncertainty in measured $\mathrm{Mn}$ content $(\mu \mathrm{g})$ : & 0.11 & 0.12 & 0.16 \\
\hline Co content of leach solution $(\mu \mathrm{g})$ : & $<0.61$ & $<0.68$ & 1.28 \\
\hline \multicolumn{4}{|l|}{ Uncertainty in measured Co content $(\mu \mathrm{g})$ : } \\
\hline Ni content of leach solution $(\mu \mathrm{g})$ : & $<1.08$ & $<1.20$ & 2.28 \\
\hline \multicolumn{4}{|l|}{ Uncertainty in measured $\mathrm{Ni}$ content $(\mu \mathrm{g})$ : } \\
\hline Ca content of leach solution $(\mu \mathrm{g})$ : & 22.60 & 8.28 & 30.88 \\
\hline Uncertainty in measured Ca content $(\mu \mathrm{g})$ : & 2.30 & 1.70 & 2.86 \\
\hline Al content of leach solution $(\mu \mathrm{g})$ : & 16.50 & 10.50 & 27.00 \\
\hline Uncertainty in measured $\mathrm{Al}$ content $(\mu \mathrm{g})$ : & 3.30 & 2.10 & 3.91 \\
\hline Ti content of leach solution $(\mu \mathrm{g})$ : & 5.40 & 8.06 & 13.46 \\
\hline Uncertainty in measured Ti content $(\mu \mathrm{g})$ : & 1.10 & 1.60 & 1.94 \\
\hline$V$ content of leach solution $(\mu \mathrm{g})$ : & 23.30 & 6.96 & 30.26 \\
\hline Uncertainty in measured $\mathrm{V}$ content $(\mu \mathrm{a})$ : & 2.30 & 0.70 & 2.40 \\
\hline
\end{tabular}

Comments

\section{Feed c. Montgomeny}

$5 / 02 / 06$

Date 
Data Report Form DRF-26A: Measurement of U Contamination and Impurities by Deconsolidation Leach

\begin{tabular}{|r|l|}
\hline Procedure: & AGR-CHAR-DAM-26 Rev. 0 \\
\hline Operator: & Fred Montgomery \\
\hline Compact lot ID: & LEU01-46T-Z \\
\hline Compact lot description: & Compacts of AGR-1 baseline coated particle composite LEU01-46T \\
\hline Compact ID numbers: & $02,04,11,28,31,61$ \\
\hline DRF filename: & IImC-agr\AGR\LeachBurnLeachILEU01-46T-Z_DRF26R0.xIs \\
\hline
\end{tabular}

\begin{tabular}{|r|c|}
\hline Mean average weight/kernel $(g):$ & $2.42 \mathrm{E}-04$ \\
\hline Uncertainty in mean average weight/kernel $(\mathrm{g}):$ & $5.96 \mathrm{E}-07$ \\
\hline Mean weight \% uranium/kernel: & 90.06 \\
\hline Standard deviation in weight \% uranium/kernel: & 0.09 \\
\hline Approximate weight uranium/kernel $(\mathrm{g}):$ & $2.18 \mathrm{E}-04$ \\
\hline Uncertainty in approx. weight uranium/kernel $(\mathrm{g}):$ & $5.76 \mathrm{E}-07$ \\
\hline
\end{tabular}

\begin{tabular}{|c|c|c|c|}
\hline & First Leach & Second Leach & Total \\
\hline Deconsolidation-leach solution ID: & L0603220101 & L0603250102 & \\
\hline Number of compacts: & \multicolumn{2}{|c|}{6} & \\
\hline \multirow{2}{*}{\multicolumn{4}{|c|}{$\begin{array}{l}\text { Volume of leach solution }(\mathrm{ml}) \text { : } \\
\text { Measured } \beta \text { activity of } 0.1 \mathrm{ml} \text { aliquot }(\mathrm{dpm}) \text { : }\end{array}$}} \\
\hline & & & \\
\hline \multicolumn{4}{|l|}{ Estimated weight of $U$ in leach solution $(\mathrm{mg})$ : } \\
\hline & & & \\
\hline \multicolumn{4}{|l|}{ Radiochemical laboratory analysis number: } \\
\hline Weight uranium leached $(\mathrm{g})$ : & $4.44 \mathrm{E}-06$ & $2.43 E-07$ & 4.68E-06 \\
\hline Uncertainty in weight uranium leached $(g)$ : & $4.40 E-07$ & $2.40 E-08$ & $4.41 E-07$ \\
\hline Effective number of exposed kernels: & 0.0 & 0.0 & 0.0 \\
\hline Uncertainty in effective number of exposed kernels: & 0.0 & 0.0 & 0.0 \\
\hline & & & \\
\hline Fe content of leach solution $(\mu \mathrm{g})$ : & 3.46 & 2.18 & 5.64 \\
\hline Uncertainty in measured Fe content $(\mu \mathrm{g})$ : & 0.69 & 0.44 & 0.82 \\
\hline $\mathrm{Cr}$ content of leach solution $(\mu \mathrm{g})$ : & $<2.69$ & $<2.37$ & 5.06 \\
\hline \multicolumn{4}{|l|}{ Uncertainty in measured $\mathrm{Cr}$ content $(\mu \mathrm{q})$ : } \\
\hline Mn content of leach solution $(\mu \mathrm{g})$ : & 0.95 & 0.84 & 1.79 \\
\hline Uncertainty in measured Mn content $(\mu \mathrm{g})$ : & 0.19 & 0.17 & 0.25 \\
\hline Co content of leach solution $(\mu \mathrm{g})$ : & $<1.07$ & $<0.95$ & 2.02 \\
\hline \multicolumn{4}{|l|}{ Uncertainty in measured Co content $(\mu \mathrm{g})$ : } \\
\hline Ni content of leach solution $(\mu \mathrm{g})$ : & $<1.90$ & $<1.68$ & 3.58 \\
\hline \multicolumn{4}{|l|}{ Uncertainty in measured Ni content $(\mu q)$ : } \\
\hline Ca content of leach solution $(\mu \mathrm{g})$ : & 28.90 & 5.53 & 34.43 \\
\hline Uncertainty in measured Ca content $(\mu \mathrm{g})$ : & 2.90 & 1.10 & 3.10 \\
\hline Al content of leach solution $(\mu \mathrm{g})$ : & 14.60 & 11.30 & 25.90 \\
\hline Uncertainty in measured $\mathrm{Al}$ content $(\mu \mathrm{g})$ : & 2.90 & 2.30 & 3.70 \\
\hline Ti content of leach solution $(\mu \mathrm{g})$ : & 18.10 & 18.30 & 36.40 \\
\hline Uncertainty in measured Ti content $(\mu \mathrm{g})$ : & 1.80 & 1.80 & 2.55 \\
\hline$V$ content of leach solution $(\mu \mathrm{g})$ : & 47.70 & 10.00 & 57.70 \\
\hline Uncertainty in measured $\mathrm{V}$ content (ua): & 4.80 & 1.00 & 4.90 \\
\hline
\end{tabular}

Comments

\section{Feed c. montgomery}


Data Report Form DRF-26A: Measurement of U Contamination and Impurities by Deconsolidation Leach

\begin{tabular}{|r|l|}
\hline Procedure: & AGR-CHAR-DAM-26 Rev. 0 \\
\hline Operator: & Fred Montgomery \\
\hline Compact lot ID: & LEU01-46T-Z \\
\hline Compact lot description: & Compacts of AGR-1 baseline coated particle composite LEU01-46T \\
\hline Compact ID numbers: & $25,41,51,63,70,73$ \\
\hline DRF filename: & $\backslash 1$ mc-agr\AGR\LeachBurnLeach \LEU01-46T-Z_DRF26R0.xIs \\
\hline
\end{tabular}

\begin{tabular}{|r|c|}
\hline Mean average weight/kernel (g): & $2.42 \mathrm{E}-04$ \\
\hline Uncertainty in mean average weight/kernel (g): & $5.96 \mathrm{E}-07$ \\
\hline Mean weight \% uranium/kernel: & 90.06 \\
\hline Standard deviation in weight \% uranium/kernel: & 0.09 \\
\hline Approximate weight uranium/kernel (g): & $2.18 \mathrm{E}-04$ \\
\hline Uncertainty in approx. weight uranium/kernel (g): & $5.76 \mathrm{E}-07$ \\
\hline
\end{tabular}

\begin{tabular}{|c|c|c|c|}
\hline - & First Leach & Second Leach & Total \\
\hline Deconsolidation-leach solution ID: & L0603250201 & L0603280102 & \\
\hline Number of compacts: & \multicolumn{2}{|c|}{6} & \\
\hline \multicolumn{4}{|l|}{ Volume of leach solution $(\mathrm{ml})$ : } \\
\hline \multirow{2}{*}{\multicolumn{4}{|c|}{$\begin{array}{r}\text { Measured } \beta \text { activity of } 0.1 \mathrm{ml} \text { aliquot }(\mathrm{dpm}) \text { : } \\
\text { Estimated weight of } U \text { in leach solution }(\mathrm{mg}) \text { : }\end{array}$}} \\
\hline & & & \\
\hline \multirow{2}{*}{\multicolumn{4}{|c|}{ Radiochemical laboratory analysis number: }} \\
\hline & & & \\
\hline Weight uranium leached $(g)$ : & 1.17E-06 & $1.00 \mathrm{E}-07$ & $1.27 \mathrm{E}-06$ \\
\hline Uncertainty in weight uranium leached $(g)$ : & $1.20 \mathrm{E}-07$ & $1.00 \mathrm{E}-08$ & $1.20 \mathrm{E}-07$ \\
\hline Effective number of exposed kernels: & 0.0 & 0.0 & 0.0 \\
\hline Uncertainty in effective number of exposed kernels: & 0.0 & 0.0 & 0.0 \\
\hline \multicolumn{4}{|l|}{ 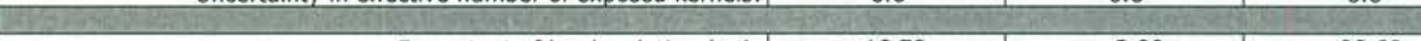 } \\
\hline Fe content of leach solution $(\mu \mathrm{g})$ : & 16.70 & 3.99 & 20.69 \\
\hline Uncertainty in measured Fe content $(\mu \mathrm{g})$ : & 1.70 & 0.80 & 1.88 \\
\hline $\mathrm{Cr}$ content of leach solution $(\mu \mathrm{g})$ : & $<2.12$ & $<2.37$ & 4.49 \\
\hline \multicolumn{4}{|l|}{ Uncertainty in measured $\mathrm{Cr}$ content $(\mu \mathrm{g})$ : } \\
\hline Mn content of leach solution $(\mu \mathrm{g})$ : & 0.81 & 0.84 & 1.65 \\
\hline Uncertainty in measured Mn content $(\mu \mathrm{g})$ : & 0.16 & 0.17 & 0.23 \\
\hline Co content of leach solution $(\mu \mathrm{g})$ : & $<0.85$ & $<0.95$ & 1.79 \\
\hline \multicolumn{4}{|l|}{ Uncertainty in measured Co content $(\mu \mathrm{g})$ : } \\
\hline Ni content of leach solution $(\mu \mathrm{g})$ : & $<1.50$ & $<1.68$ & 3.18 \\
\hline \multicolumn{4}{|l|}{ Uncertainty in measured Ni content $(\mu \mathrm{g})$ : } \\
\hline Ca content of leach solution $(\mu \mathrm{g})$ : & 20.10 & 5.09 & 25.19 \\
\hline Uncertainty in measured Ca content $(\mu \mathrm{g})$ : & 2.00 & 1.00 & 2.24 \\
\hline Al content of leach solution $(\mu \mathrm{g})$ : & $<8.18$ & 13.40 & 21.58 \\
\hline Uncertainty in measured $\mathrm{Al}$ content $(\mu \mathrm{g})$ : & & 2.70 & 2.70 \\
\hline Ti content of leach solution $(\mu \mathrm{g})$ : & 11.70 & 12.80 & 24.50 \\
\hline Uncertainty in measured $\mathrm{Ti}$ content $(\mu \mathrm{q})$ : & 2.30 & 2.60 & 3.47 \\
\hline$V$ content of leach solution $(\mu \mathrm{g})$ : & 45.90 & 10.80 & 56.70 \\
\hline Uncertainty in measured $\mathrm{V}$ content $(\mu \mathrm{q})$ : & 4.60 & 1.10 & 4.73 \\
\hline
\end{tabular}

Comments

\section{Fied c. Montgomay}


Data Report Form DRF-26B: Measurement of SiC Burn-Leach Defects and Impurities by Burn-Leach

\begin{tabular}{|r|l|}
\hline Procedure: & AGR-CHAR-DAM-26 Rev. 0 \\
\hline Operator: & Fred Montgomery \\
\hline Compact lot ID: & LEU01-46T-Z \\
\hline Compact lot description: & Compacts of AGR-1 baseline coated particle composite LEU01-46T \\
\hline Compact ID numbers: & $10,13,66$ \\
\hline DRF filename: & $\backslash 1$ mc-agr\AGR\LeachBurnLeach \LEU01-46T-Z_DRF26R0.xIs \\
\hline
\end{tabular}

\begin{tabular}{|r|c|}
\hline Mean average weight/kernel $(\mathrm{g}):$ & $2.42 \mathrm{E}-04$ \\
\hline Uncertainty in mean average weight/kernel $(\mathrm{g}):$ & $5.96 \mathrm{E}-07$ \\
\hline Mean weight \% uranium/kernel: & 90.06 \\
\hline Standard deviation in weight \% uranium/kernel: & 0.09 \\
\hline Approximate weight uranium $/$ kernel $(\mathrm{g}):$ & $2.18 \mathrm{E}-04$ \\
\hline Uncertainty in approx. weight uranium/kernel $(\mathrm{g}):$ & $5.76 \mathrm{E}-07$ \\
\hline
\end{tabular}

\begin{tabular}{|c|c|c|c|}
\hline & First Leach & Second Leach & Total \\
\hline Burn-leach solution ID: & $\mathrm{B} 0603280201$ & $\mathrm{~B} 0603300202$ & \\
\hline Number of compacts: & \multicolumn{2}{|c|}{3} & \\
\hline \multicolumn{4}{|l|}{ Volume of leach solution $(\mathrm{ml})$ : } \\
\hline \multicolumn{4}{|l|}{ Measured $\beta$ activity of $0.1 \mathrm{ml}$ aliquot (dpm): } \\
\hline \multirow{2}{*}{\multicolumn{4}{|c|}{ Estimated weight of $U$ in leach solution $(\mathrm{mg})$ : }} \\
\hline & & & \\
\hline \multicolumn{4}{|l|}{ Radiochemical laboratory analysis number: } \\
\hline Weight uranium leached $(g)$ : & $2.29 E-04$ & $3.86 \mathrm{E}-07$ & $2.29 \mathrm{E}-04$ \\
\hline Uncertainty in weight uranium leached $(g)$ : & $2.30 \mathrm{E}-05$ & $3.90 \mathrm{E}-08$ & $2.30 E-05$ \\
\hline Number of leached kernels: & 1.1 & 0.0 & 1.1 \\
\hline Uncertainty in number of leached kernels: & 0.1 & 0.0 & 0.1 \\
\hline \multicolumn{4}{|c|}{ 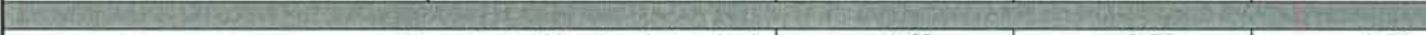 } \\
\hline $\begin{array}{r}\text { Fe content of leach solution }(\mu \mathrm{g}) \text { : } \\
\text { Uncertainty in measured Fe content }(\mu \mathrm{g}) \text { : }\end{array}$ & 1.63 & $<0.26$ & 1.89 \\
\hline $\begin{array}{r}\text { Uncertainty in measured Fe content }(\mu \mathrm{g}): \\
\mathrm{Cr} \text { content of leach solution }(\mu \mathrm{g}):\end{array}$ & 0.16 & & 0.16 \\
\hline \multirow{2}{*}{\multicolumn{4}{|c|}{ Uncertainty in measured $\mathrm{Cr}$ content $(\mu \mathrm{g})$ : }} \\
\hline Mn content of leach solution $(\mu \mathrm{g})$ : & & & \\
\hline \multicolumn{4}{|l|}{ Uncertainty in measured $\mathrm{Mn}$ content $(\mu \mathrm{g})$ : } \\
\hline Co content of leach solution $(\mu \mathrm{g})$ : & $<0.28$ & $<0.28$ & 0.56 \\
\hline \multicolumn{4}{|l|}{ Uncertainty in measured Co content $(\mu \mathrm{g})$ : } \\
\hline Ni content of leach solution $(\mu \mathrm{g})$ : & $<0.50$ & $<0.50$ & 1.00 \\
\hline \multicolumn{4}{|l|}{ Uncertainty in measured $\mathrm{Ni}$ content $(\mu \mathrm{g})$ : } \\
\hline Ca content of leach solution $(\mu g)$ : & 11.00 & 3.11 & 14.11 \\
\hline Uncertainty in measured Ca content $(\mu \mathrm{g})$ : & 1.10 & 0.62 & 1.26 \\
\hline Al content of leach solution $(\mu \mathrm{g})$ : & 11.40 & $<2.73$ & 14.13 \\
\hline Uncertainty in measured $\mathrm{Al}$ content $(\mu \mathrm{g})$ : & 2.30 & & 2.30 \\
\hline Ti content of leach solution $(\mu \mathrm{g})$ : & 1.36 & 3.60 & 4.96 \\
\hline Uncertainty in measured Ti content $(\mu \mathrm{g})$ : & 0.27 & 0.72 & 0.77 \\
\hline$V$ content of leach solution $(\mu g)$ : & 20.80 & 1.71 & 22.51 \\
\hline Uncertainty in measured $\mathrm{V}$ content ( $\mathrm{uq}$ ): & 2.10 & 0.34 & 2.13 \\
\hline
\end{tabular}

Comments 
Data Report Form DRF-26B: Measurement of SiC Burn-Leach Defects and Impurities by Burn-Leach

\begin{tabular}{|c|c|}
\hline Procedure: & AGR-CHAR-DAM-26 Rev. 0 \\
\hline Operator: & Fred Montgomery \\
\hline Compact lot ID: & LEU $01-46 T-Z$ \\
\hline Compact lot description: & Compacts of AGR-1 baseline coated particle composite LEU01-46T \\
\hline Compact ID numbers: & $40,48,64$ \\
\hline DRF filename: & IImc-agr\AGR\LeachBurnLeach\LEU01-46T-Z_DRF26R0.xIs \\
\hline
\end{tabular}

\begin{tabular}{|r|c|}
\hline Mean average weight/kernel $(g):$ & $2.42 \mathrm{E}-04$ \\
\hline Uncertainty in mean average weight/kernel $(\mathrm{g}):$ & $5.96 \mathrm{E}-07$ \\
\hline Mean weight \% uranium/kernel: & 90.06 \\
\hline Standard deviation in weight \% uranium/kernel: & 0.09 \\
\hline Approximate weight uranium/kernel $(g):$ & $2.18 \mathrm{E}-04$ \\
\hline Uncertainty in approx. weight uranium/kernel $(\mathrm{g}):$ & $5.76 \mathrm{E}-07$ \\
\hline
\end{tabular}

\begin{tabular}{|c|c|c|c|}
\hline & First Leach & Second Leach & Total \\
\hline Burn-leach solution ID: & B0603280301 & B0603300302 & \\
\hline Number of compacts: & \multicolumn{2}{|l|}{ 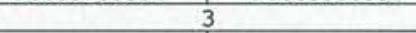 } & \\
\hline \multicolumn{4}{|l|}{ Volume of leach solution $(\mathrm{ml})$ : } \\
\hline \multirow{2}{*}{\multicolumn{4}{|c|}{$\begin{array}{l}\text { Measured } \beta \text { activity of } 0.1 \mathrm{ml} \text { aliquot }(\mathrm{dpm}) \text { : } \\
\text { Estimated weight of } U \text { in leach solution }(\mathrm{mg}) \text { : }\end{array}$}} \\
\hline & & & \\
\hline \multicolumn{4}{|l|}{ Radiochemical laboratory analysis number: } \\
\hline Weight uranium leached $(g)$ : & $2.47 E-04$ & $1.27 \mathrm{E}-07$ & 2.47E-04 \\
\hline Uncertainty in weight uranium leached $(g)$ : & $2.50 E-05$ & $1.30 \mathrm{E}-08$ & $2.50 E-05$ \\
\hline Number of leached kernels: & 1.1 & 0.0 & 1.1 \\
\hline Uncertainty in number of leached kernels: & 0.1 & 0.0 & 0.1 \\
\hline \multicolumn{4}{|l|}{ 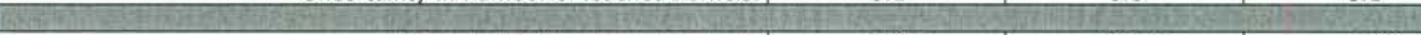 } \\
\hline Fe content of leach solution $(\mu \mathrm{g})$ : & 1.82 & $<0.26$ & 2.08 \\
\hline Uncertainty in measured $\mathrm{Fe}$ content $(\mu \mathrm{g})$ : & 0.18 & & 0.18 \\
\hline $\mathrm{Cr}$ content of leach solution $(\mu \mathrm{g})$ : & $<0.71$ & $<0.71$ & 1.41 \\
\hline \multicolumn{4}{|l|}{ Uncertainty in measured $\mathrm{Cr}$ content $(\mu \mathrm{g})$ : } \\
\hline Mn content of leach solution $(\mu \mathrm{g})$ : & $<0.10$ & $<0.10$ & 0.20 \\
\hline \multicolumn{4}{|l|}{ Uncertainty in measured Mn content ( $\mu \mathrm{g})$ : } \\
\hline Co content of leach solution $(\mu \mathrm{g})$ : & $<0.28$ & $<0.28$ & 0.56 \\
\hline \multicolumn{4}{|l|}{ Uncertainty in measured Co content $(\mu \mathrm{g})$ : } \\
\hline Ni content of leach solution $(\mu \mathrm{g})$ : & 0.76 & $<0.50$ & 1.26 \\
\hline Uncertainty in measured Ni content $(\mu \mathrm{q})$ : & 0.15 & & 0.15 \\
\hline Ca content of leach solution $(\mu \mathrm{q})$ : & 22.10 & 4.85 & 26.95 \\
\hline Uncertainty in measured Ca content $(\mu \mathrm{g})$ : & 2.20 & 0.97 & 2.40 \\
\hline Al content of leach solution $(\mu \mathrm{g})$ : & 12.10 & $<2.73$ & 14.83 \\
\hline Uncertainty in measured $\mathrm{Al}$ content $(\mu \mathrm{g})$ : & 2.40 & & 2.40 \\
\hline Ti content of leach solution $(\mu \mathrm{g})$ : & 11.90 & $<0.86$ & 12.76 \\
\hline Uncertainty in measured Ti content $(\mu \mathrm{g})$ : & 1.20 & & 1.20 \\
\hline$V$ content of leach solution $(\mu \mathrm{g})$ : & 20.10 & 1.32 & 21.42 \\
\hline Uncertainty in measured $\mathrm{V}$ content (uq): & 2.00 & 0.26 & 2.02 \\
\hline
\end{tabular}

\section{Comments}

\section{Fied c. Montgomery}


Data Report Form DRF-26B: Measurement of SiC Burn-Leach Defects and Impurities by Burn-Leach

\begin{tabular}{|c|c|}
\hline Procedure: & AGR-CHAR-DAM-26 Rev. 0 \\
\hline Operator: & Fred Montgomery \\
\hline Compact lot ID: & LEU01-46T-Z \\
\hline Compact lot description: & Compacts of AGR-1 baseline coated particle composite LEU01-46T \\
\hline Compact ID numbers: & $35,54,59$ \\
\hline DRF filename: & IImc-agr\AGR\LeachBurnLeach\LEU01-46T-Z_DRF26R0.xIs \\
\hline
\end{tabular}

\begin{tabular}{|r|c|}
\hline Mean average weight/kernel $(g):$ & $2.42 \mathrm{E}-04$ \\
\hline Uncertainty in mean average weight/kernel $(g):$ & $5.96 \mathrm{E}-07$ \\
\hline Mean weight \% uranium/kernel: & 90.06 \\
\hline Standard deviation in weight \% uranium/kernel: & 0.09 \\
\hline Approximate weight uranium/kernel $(g):$ & $2.18 \mathrm{E}-04$ \\
\hline Uncertainty in approx. weight uranium/kernel $(g):$ & $5.76 \mathrm{E}-07$ \\
\hline
\end{tabular}

\begin{tabular}{|c|c|c|c|}
\hline & First Leach & Second Leach & Total \\
\hline Burn-leach solution ID: & B0603310101 & B0604030302 & \\
\hline Number of compacts: & \multicolumn{2}{|l|}{$x_{0}=0$} & \\
\hline \multicolumn{4}{|l|}{ Volume of leach solution $(\mathrm{ml})$ : } \\
\hline \multirow{2}{*}{\multicolumn{4}{|c|}{$\begin{array}{r}\text { Measured } \beta \text { activity of } 0.1 \mathrm{ml} \text { aliquot }(\mathrm{dpm}) \text { : } \\
\text { Estimated weight of } U \text { in leach solution }(\mathrm{mg}) \text { : }\end{array}$}} \\
\hline & & & \\
\hline \multicolumn{4}{|l|}{ Radiochemical laboratory analysis number: } \\
\hline Weight uranium leached $(g)$ : & $2.14 \mathrm{E}-07$ & $6.70 \mathrm{E}-09$ & $2.21 \mathrm{E}-07$ \\
\hline Uncertainty in weight uranium leached $(\mathrm{g})$ : & $2.10 \mathrm{E}-08$ & $6.70 \mathrm{E}-10$ & $2.10 \mathrm{E}-08$ \\
\hline Number of leached kernels: & 0.0 & 0.0 & 0.0 \\
\hline Uncertainty in number of leached kernels: & 0.0 & 0.0 & 0.0 \\
\hline \multicolumn{4}{|l|}{ 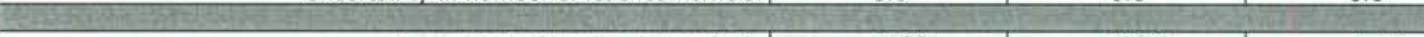 } \\
\hline Fe content of leach solution $(\mu \mathrm{g})$ : & 1.20 & $<0.26$ & 1.46 \\
\hline Uncertainty in measured Fe content $(\mu q)$ : & 0.22 & & 0.22 \\
\hline $\mathrm{Cr}$ content of leach solution $(\mu \mathrm{q})$ : & $<0.71$ & $<0.71$ & 1.41 \\
\hline \multicolumn{4}{|l|}{ Uncertainty in measured $\mathrm{Cr}$ content $(\mu \mathrm{g})$ : } \\
\hline Mn content of leach solution $(\mu \mathrm{g})$ : & $<0.10$ & 0.27 & 0.37 \\
\hline Uncertainty in measured $\mathrm{Mn}$ content $(\mu \mathrm{g})$ : & & 0.05 & 0.05 \\
\hline Co content of leach solution $(\mu \mathrm{g})$ : & $<0.28$ & $<0.28$ & 0.56 \\
\hline \multicolumn{4}{|l|}{ Uncertainty in measured Co content $(\mu \mathrm{q})$ : } \\
\hline Ni content of leach solution $(\mu \mathrm{g})$ : & 1.16 & $<0.50$ & 1.66 \\
\hline Uncertainty in measured Ni content $(\mu \mathrm{g})$ : & 0.23 & & 0.23 \\
\hline Ca content of leach solution $(\mu \mathrm{g})$ : & 20.80 & 1.32 & 22.12 \\
\hline Uncertainty in measured Ca content $(\mu \mathrm{g})$ : & 2.10 & 0.26 & 2.12 \\
\hline Al content of leach solution $(\mu \mathrm{g})$ : & 5.41 & $<2.73$ & 8.14 \\
\hline Uncertainty in measured $\mathrm{Al}$ content $(\mu \mathrm{g})$ : & 1.10 & & 1.10 \\
\hline Ti content of leach solution $(\mu \mathrm{g})$ : & 10.80 & 3.29 & 14.09 \\
\hline Uncertainty in measured $\mathrm{Ti}$ content $(\mu \mathrm{g})$ : & 1.10 & 0.66 & 1.28 \\
\hline$V$ content of leach solution $(\mu \mathrm{g})$ : & 26.60 & 0.94 & 27.54 \\
\hline Uncertainty in measured $\mathrm{V}$ content $(\mathrm{uq})$ : & 2.70 & 0.19 & 2.71 \\
\hline
\end{tabular}

\section{Comments}

\section{Feed c. Muntgomery


Data Report Form DRF-26B: Measurement of SiC Burn-Leach Defects and Impurities by Burn-Leach

\begin{tabular}{|c|c|}
\hline Procedure: & AGR-CHAR-DAM-26 Rev. 0 \\
\hline Operator: & Fred Montgomery \\
\hline Compact lot ID: & LEU 0 1-46T-Z \\
\hline Compact lot description: & Compacts of AGR-1 baseline coated particle composite LEU01-46T \\
\hline Compact ID numbers: & $08,26,75$ \\
\hline DRF filename: & IImc-agr|AGR\LeachBurnLeach|LEU01-46T-Z_DRF26R0.xIs \\
\hline
\end{tabular}

\begin{tabular}{|r|c|}
\hline Mean average weight/kernel $(\mathrm{g}):$ & $2.42 \mathrm{E}-04$ \\
\hline Uncertainty in mean average weight/kernel $(\mathrm{g}):$ & $5.96 \mathrm{E}-07$ \\
\hline Mean weight \% uranium/kernel: & 90.06 \\
\hline Standard deviation in weight \% uranium/kernel: & 0.09 \\
\hline Approximate weight uranium/kernel $(g):$ & $2.18 \mathrm{E}-04$ \\
\hline Uncertainty in approx. weight uranium/kernel $(\mathrm{g}):$ & $5.76 \mathrm{E}-07$ \\
\hline
\end{tabular}

\begin{tabular}{|c|c|c|c|}
\hline & First Leach & Second Leach & Total \\
\hline Burn-leach solution ID: & $\mathrm{B} 0603310201$ & B0604030402 & \\
\hline Number of compacts: & \multicolumn{2}{|c|}{3} & \\
\hline \multicolumn{4}{|l|}{ Volume of leach solution $(\mathrm{ml})$ : } \\
\hline Measured $\beta$ activity of $0.1 \mathrm{ml}$ aliquot (dpm): & & & \\
\hline \multicolumn{4}{|l|}{ Estimated weight of $U$ in leach solution $(\mathrm{mg})$ : } \\
\hline \multirow{2}{*}{\multicolumn{4}{|c|}{ Radiochemical laboratory analysis number: }} \\
\hline & & & \\
\hline Weight uranium leached $(g)$ : & $3.97 \mathrm{E}-07$ & $2.53 E-08$ & $4.22 E-07$ \\
\hline Uncertainty in weight uranium leached $(g)$ : & $4.00 E-08$ & $2.50 \mathrm{E}-09$ & 4.01E-08 \\
\hline Number of leached kernels: & 0.0 & 0.0 & 0.0 \\
\hline Uncertainty in number of leached kernels: & 0.0 & 0.0 & 0.0 \\
\hline \multicolumn{4}{|l|}{ 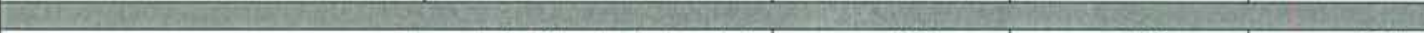 } \\
\hline Fe content of leach solution $(\mu \mathrm{q})$ : & 4.15 & 1.32 & 5.47 \\
\hline Uncertainty in measured Fe content $(\mu \mathrm{q})$ : & 0.42 & 0.13 & 0.44 \\
\hline $\mathrm{Cr}$ content of leach solution $(\mu \mathrm{g})$ : & $<0.71$ & $<0.71$ & 1.41 \\
\hline \multicolumn{4}{|l|}{ Uncertainty in measured $\mathrm{Cr}$ content $(\mu \mathrm{g})$ : } \\
\hline Mn content of leach solution $(\mu \mathrm{g})$ : & $<0.10$ & $<0.10$ & 0.20 \\
\hline \multicolumn{4}{|l|}{ Uncertainty in measured Mn content $(\mu \mathrm{g})$ : } \\
\hline Co content of leach solution $(\mu \mathrm{q})$ : & $<0.28$ & $<0.28$ & 0.56 \\
\hline \multicolumn{4}{|l|}{ Uncertainty in measured Co content $(\mu \mathrm{g})$ : } \\
\hline Ni content of leach solution $(\mu \mathrm{g})$ : & $<0.50$ & $<0.50$ & 1.00 \\
\hline \multicolumn{4}{|l|}{ Uncertainty in measured Ni content $(\mu \mathrm{g})$ : } \\
\hline Ca content of leach solution $(\mu \mathrm{g})$ : & 15.40 & 3.22 & 18.62 \\
\hline Uncertainty in measured Ca content $(\mu \mathrm{g})$ : & 1.50 & 0.64 & 1.63 \\
\hline Al content of leach solution $(\mu \mathrm{g})$ : & $<2.73$ & 2.89 & 5.62 \\
\hline Uncertainty in measured $\mathrm{Al}$ content $(\mu \mathrm{g})$ : & & 0.58 & 0.58 \\
\hline Ti content of leach solution $(\mu \mathrm{g})$ : & 8.88 & 7.38 & 16.26 \\
\hline Uncertainty in measured $\mathrm{Ti}$ content $(\mu \mathrm{g})$ : & 0.89 & 0.74 & 1.16 \\
\hline$V$ content of leach solution $(\mu \mathrm{g})$ : & 26.40 & 1.20 & 27.60 \\
\hline Uncertainty in measured $\mathrm{V}$ content $(\mu \mathrm{q})$ : & 2.60 & 0.24 & 2.61 \\
\hline
\end{tabular}

Comments

\section{Feed c. Montgymery}


Data Report Form DRF-27: Counting of Particles with a Defective OPYC Layer from Deconsolidated Compacts by Visual Inspection

\begin{tabular}{|c|c|}
\hline Procedure: & AGR-CHAR-DAM-27 Rev. 0 \\
\hline Operator: & Fred Montgomery \\
\hline Compact lot ID: & LEU $1-46 T-Z$ \\
\hline Compact lot description: & Compacts of AGR-1 baseline coated particle composite LEU01-46T \\
\hline Compact ID number: & 6 \\
\hline DRF filename: & IImc-agr|AGR\DefectiveOPyC|LEU01-47T-Z_DRF27R0.xls \\
\hline
\end{tabular}

Number of particles with cracked OPyC: 0

Number of particles with partially missing OPyC: 0

Number of particles with completely missing OPyC: 0

Total number of particles with defective OPyC: 0

Comments on unusual visual characteristics of OPYC

$<5$ particles still had a thin layer of the matrix present. No agglomerates in the population.

Feed c. montgomery
$5 / 3 / 06$

Date 
ORNL/TM-2006/507, Rev. 0

Data Report Form DRF-28: Counting of Particles with Excessive Uranium Dispersion Inside SiC

\begin{tabular}{|c|c|}
\hline Procedure: & AGR-CHAR-DAM-28 Rev. 1 \\
\hline Operator: & John Hunn \\
\hline Compact lot ID: & LEU01-46T-Z \\
\hline Compact lot description: & Compacts of AGR-1 baseline coated particle composite LEU01-46T \\
\hline Compact ID numbers: & $10,13,66,40,48,64,35,54,59,08,26,75$ \\
\hline DRF filename: & 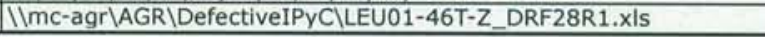 \\
\hline
\end{tabular}

Number of compacts from which particles were recovered: 12

Weight of sample of particles (g): 29.047

Number of particles in sample: 49735

Mean average weight/particle (g): $5.84 \mathrm{E}-04$

Number of particles with excessive U dispersion: 0

\section{Comments}

No defects as defined by visual standard were observed. However a number of anomalies were noted.

Particles noted with $<300 \mu \mathrm{m}$ diameter kernel: $25 / 49735$ (fraction of compact lot with this anomaly: $\leq 7.1 \mathrm{E}-4$ at $95 \%$ confidence).

Particles noted with <20 $\mu \mathrm{m}$ thick SiC: $15 / 49735$ (fraction of compact lot with this anomaly: $\leq 4.7 \mathrm{E}-4$ at $95 \%$ confidence).

Other anomalies observed included highly aspherical kernel shapes, faceted particles, and defects related to goldspots (soot inclusions).

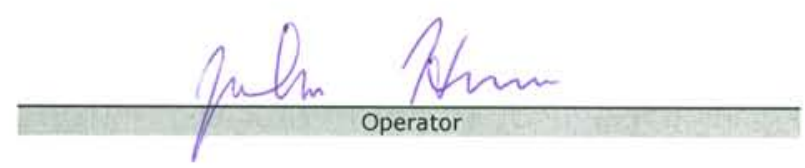

$$
7-17-06
$$

Date


ORNL/TM-2006/507, Rev. 0

\section{For Information Only}

The information in the remainder of this section is from additional characterization that was not required by the fuel product specification. 


\section{A. Images of AGR-1 baseline compact lot LEU01-46T-Z}

The compacting procedure allowed for some adjustment in the malleability of the overcoat to aid the compaction process, densify the compact, and create a smoother, less porous outer surface. Adjustments were made for the first five compacts fabricated to minimize the compact surface porosity. This resulted in some variation in the surface appearance of the first several compacts in compact lot LEU01-46T-Z. The following pictures show two compacts from lot LEU01-46TZ. The compact shown in Figure A-1 through Figure A-3 was the fourth compact fabricated. This compact showed more gaps between the overcoated particles than compacts fabricated later, when adjustments had resulted in a smoother finish. The compact shown in Figure A-4 through Figure A-6 was the twenty sixth compact fabricated.

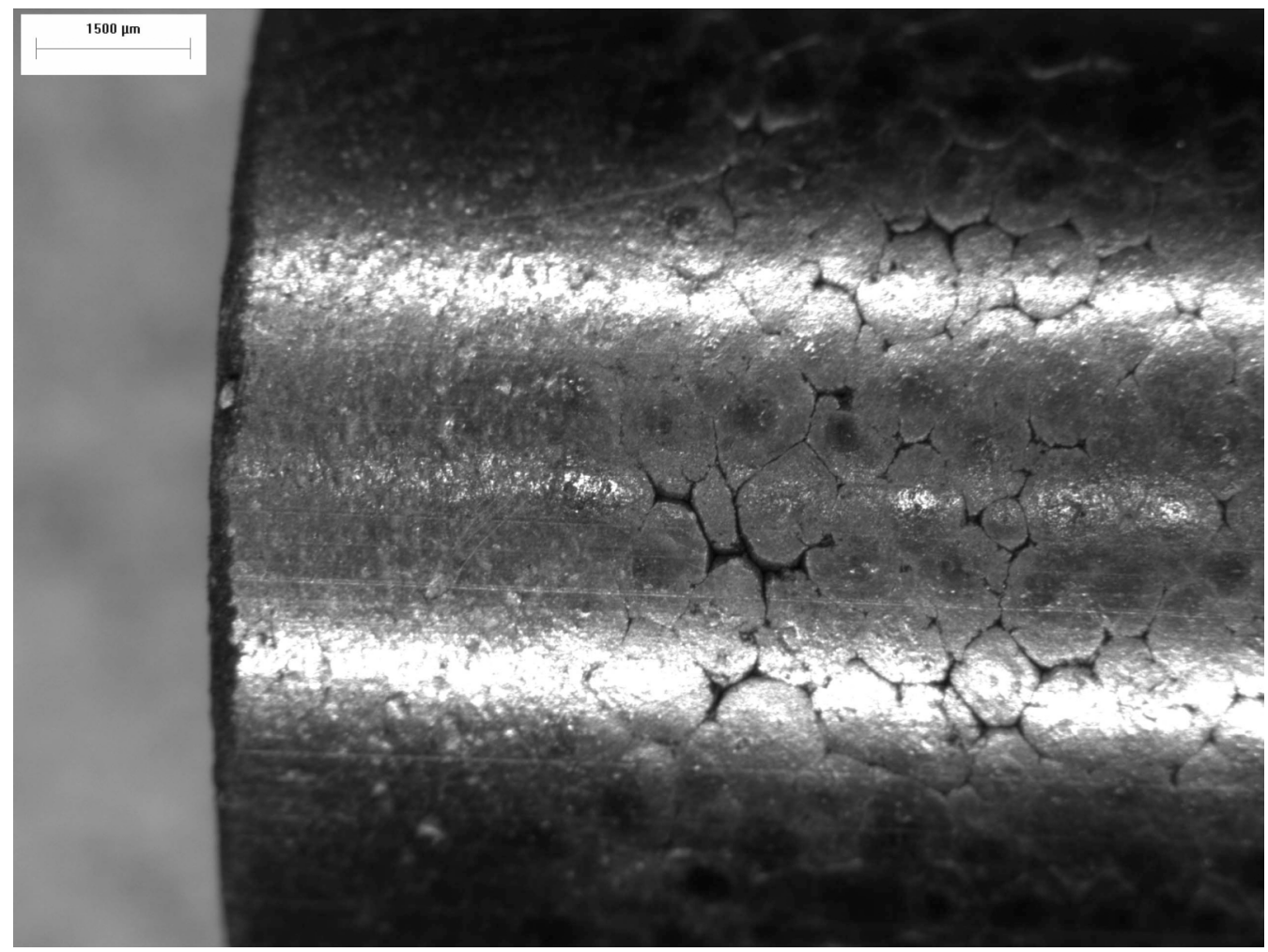

Figure A-1: Bottom of compact LEU01-46T-Z03 (4 ${ }^{\text {th }}$ compact fabricated $)$. 


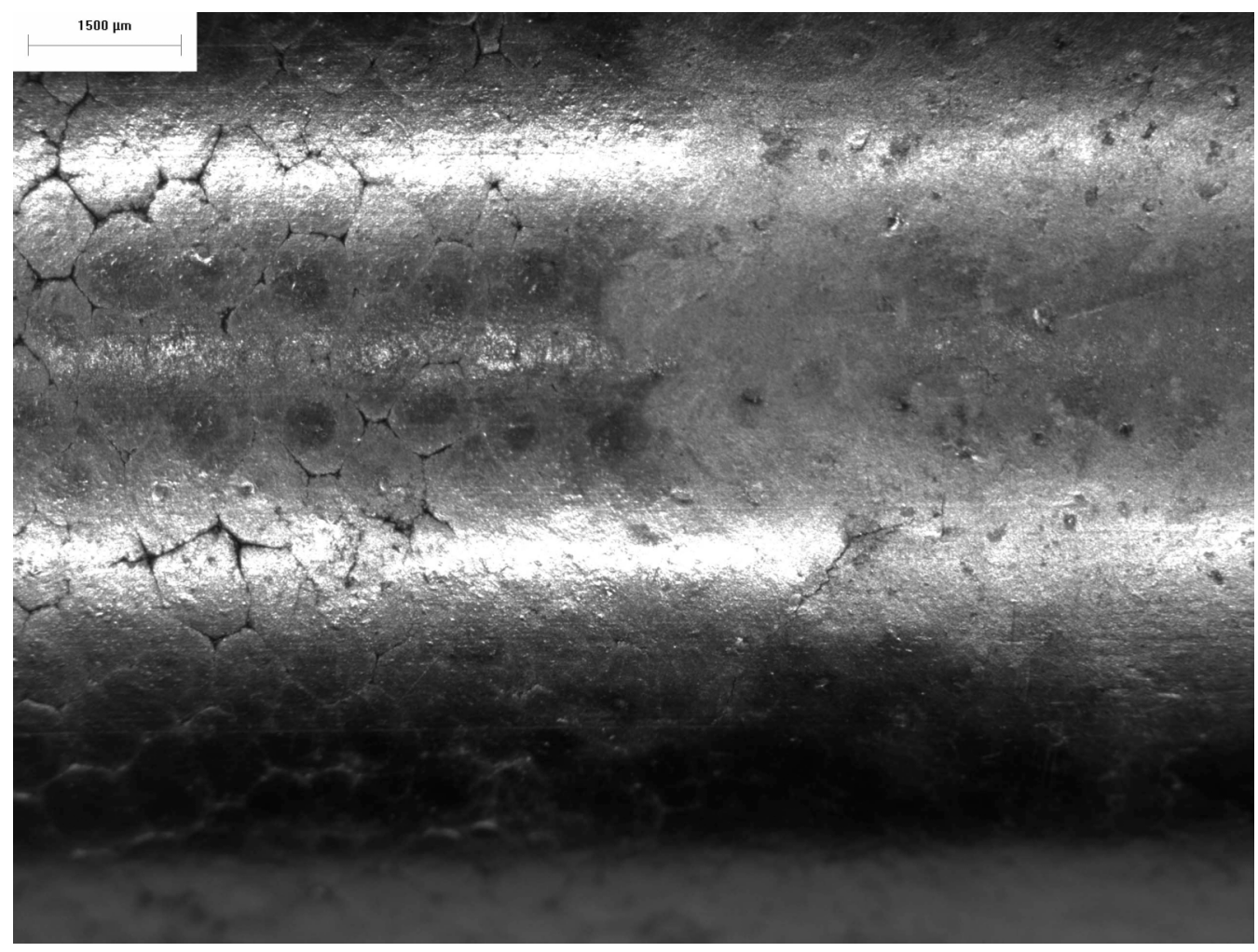

Figure A-2: Middle of compact LEU01-46T-Z03 ( $4^{\text {th }}$ compact fabricated $)$.

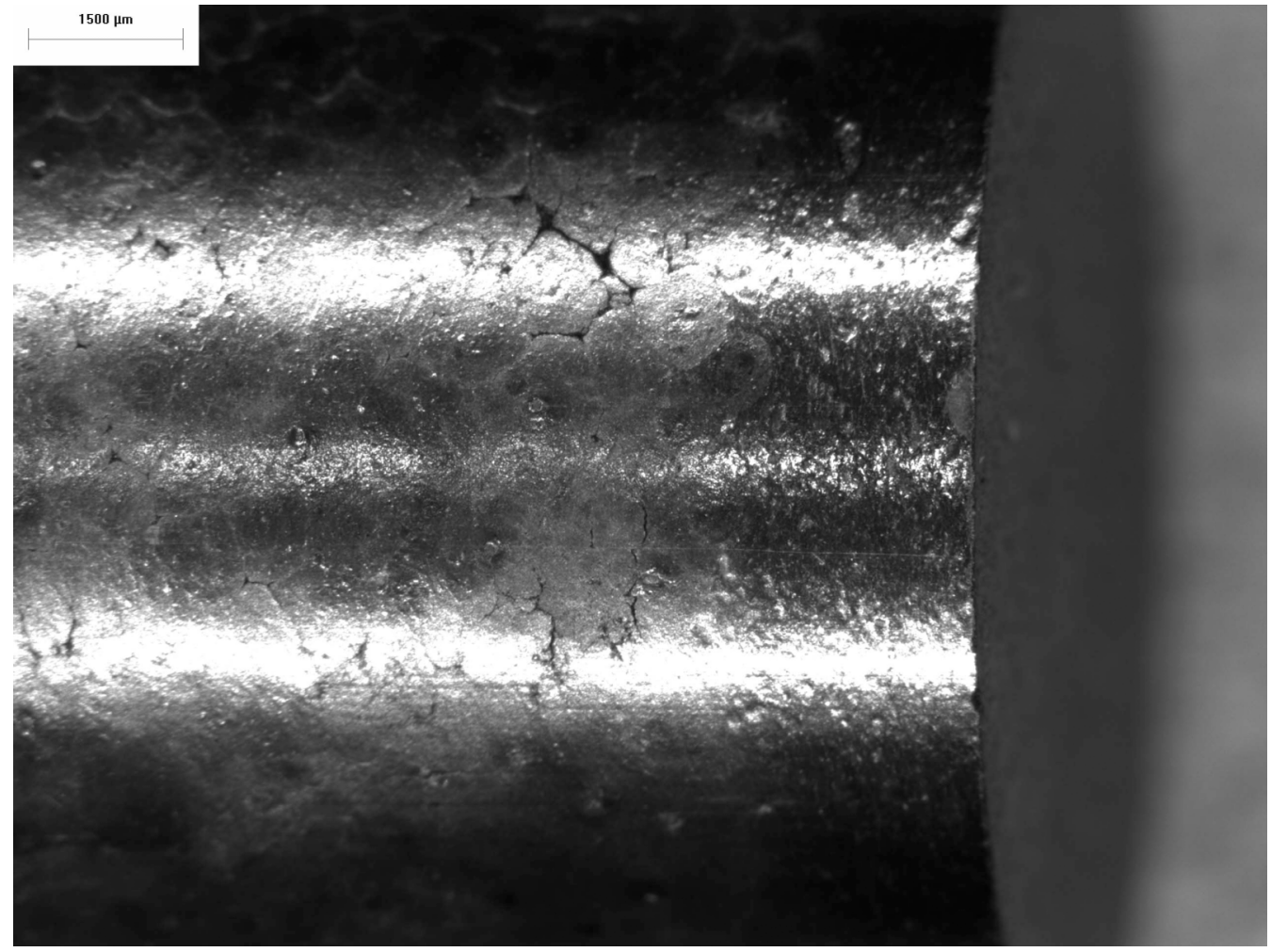

Figure A-3: Top of compact LEU01-46T-Z03 ( $4^{\text {th }}$ compact fabricated). 


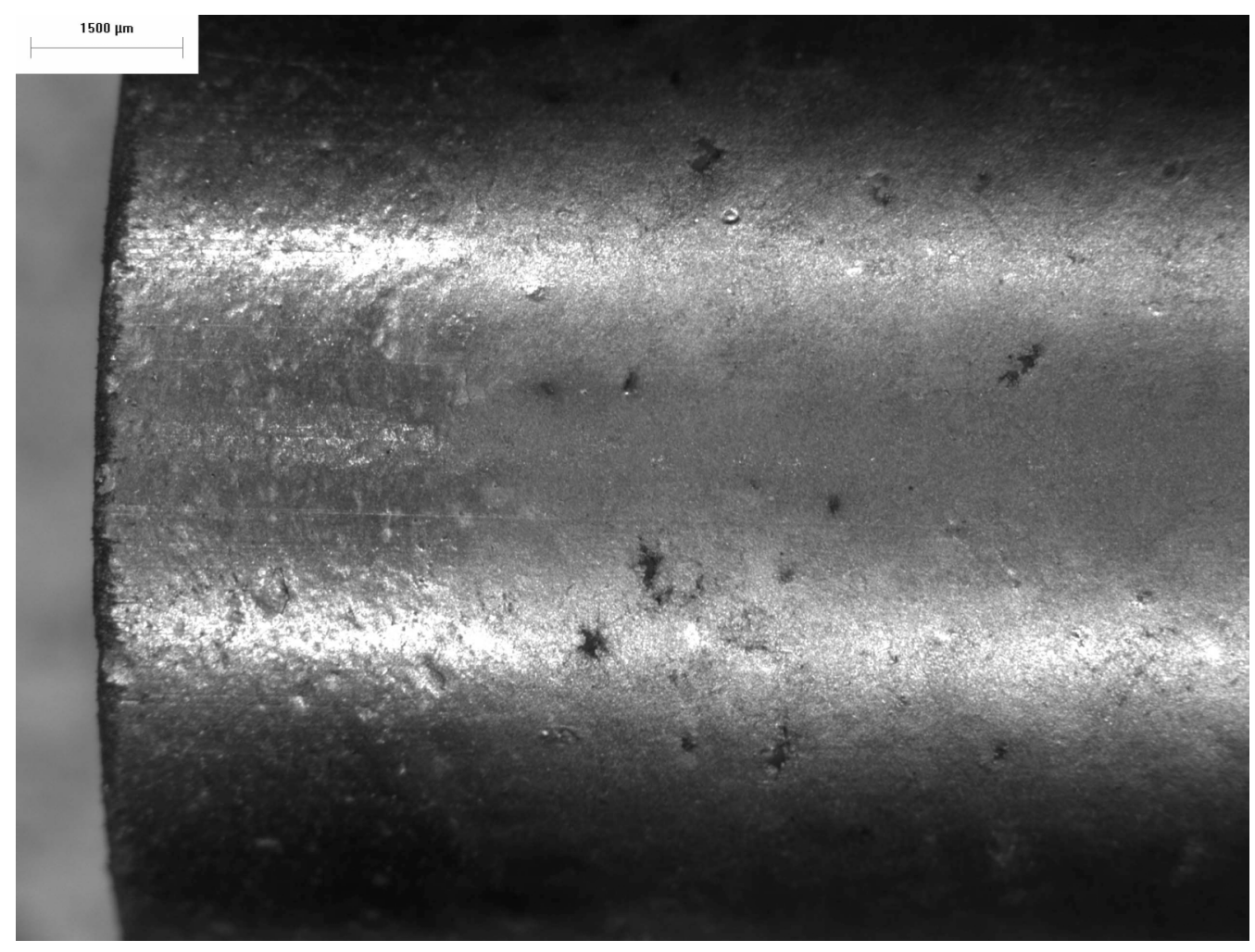

Figure A-4: Bottom of compact LEU01-46T-Z18 $\left(26^{\text {th }}\right.$ compact fabricated).

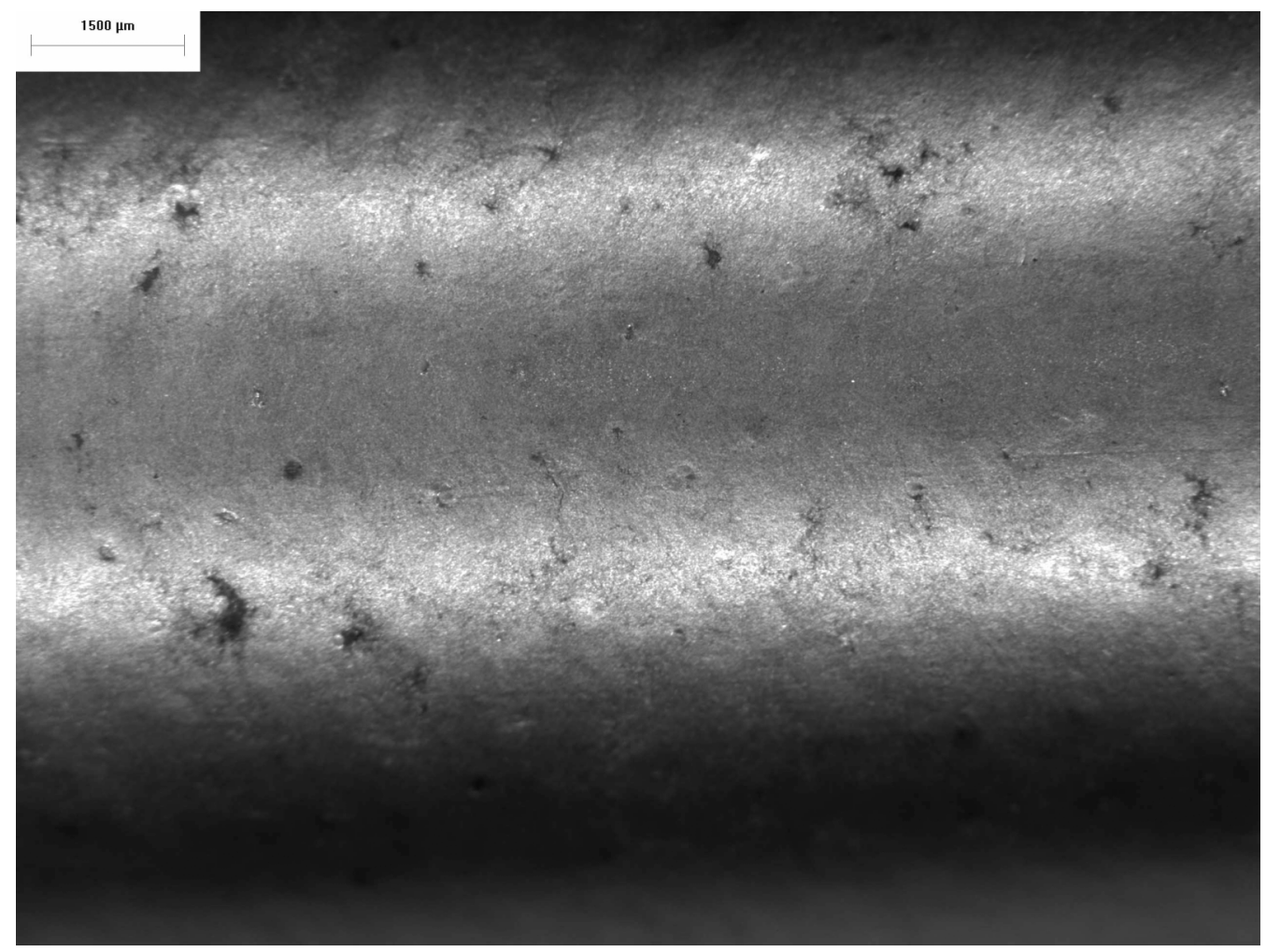

Figure A-5: Middle of compact LEU01-46T-Z18 $\left(26^{\text {th }}\right.$ compact fabricated). 


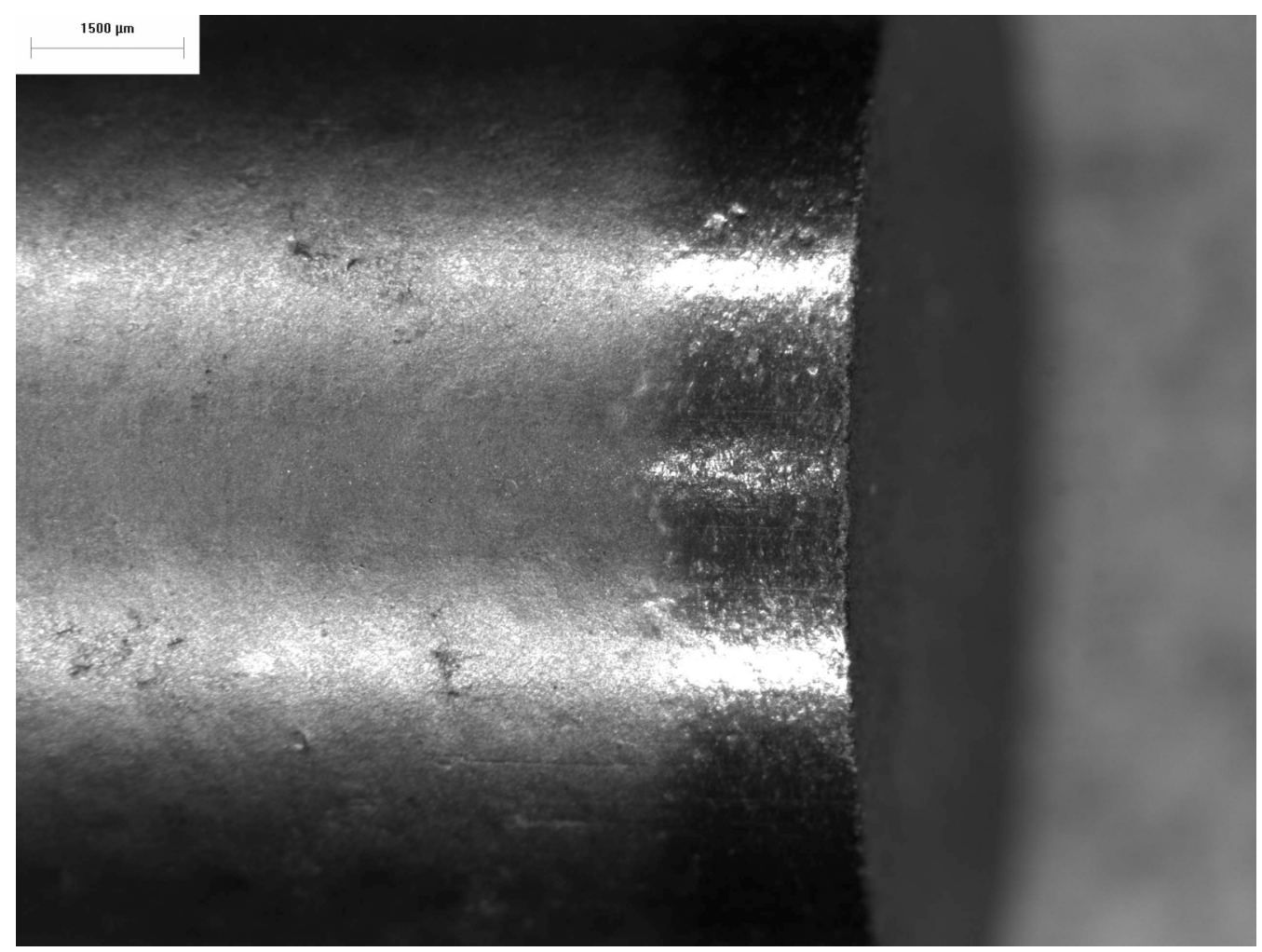

Figure A-6: Top of compact LEU01-46T-Z18 (26 ${ }^{\text {th }}$ compact fabricated). 


\section{B. Anisotropy of pyrocarbon layers after compacting}

To examine the change in pyrocarbon anisotropy during compact fabrication, particles were recovered after deconsolidation of the particles from the compact for defective OPyC analysis. After compacting, the mean anisotropy of the pyrocarbon layers was observed to increase. This increase is thought to occur during the heat treatment of the compacts at $1800^{\circ} \mathrm{C}$ for 1 hour. The mean diattenuation of the IPyC increased from $0.0074 \pm 0.0007$ to $0.0109 \pm 0.0013(1.0222 \pm 0.0021$ to $1.0326 \pm 0.0039$ in terms of effective $\mathrm{BAF}$ ). The diattenuation of the $\mathrm{OPyC}$ increased from $0.0063 \pm 0.0009$ to $0.0111 \pm 0.0009$ ( $1.0190 \pm 0.0026$ to $1.0334 \pm 0.0028$ in terms of effective BAFo). The following two DRF's contain the data for these measurements. 
Data Report Form DRF-18A: Measurement of Pyrocarbon Anisotropy using the 2-MGEM - IPyC

\begin{tabular}{|r|l|}
\hline Procedure: & AGR-CHAR-DAM-18 Rev. 1 \\
\hline Operator: & G. E. Jellison \\
\hline Mount ID: & M06062001L \\
\hline Sample ID: & LEU01-46T-Z06 \\
\hline Sample Description: & Particles deconsolidated from AGR-1 baseline compact \\
\hline Folder containing data: & $\backslash \backslash \mathrm{MC}$-agr\AGR\2-MGEM\R06071401\} $\\
{\hline}$
\end{tabular}

\begin{tabular}{|c|c|c|c|c|c|c|c|}
\hline \multirow{2}{*}{ Particle \# } & \multirow{2}{*}{$\begin{array}{c}\text { Grid } \\
\text { Position }\end{array}$} & \multicolumn{3}{|c|}{ Diattenuation } & \multicolumn{3}{|c|}{ Equivalent BAFo } \\
\hline & & Average & St. Dev. & Ave. Error & Average & St. Dev. & Ave. Error \\
\hline 1 & 4,4 & 0.0122 & 0.0032 & 0.0012 & 1.0366 & 0.0096 & 0.0036 \\
\hline 2 & 4,5 & 0.0104 & 0.0032 & 0.0012 & 1.0312 & 0.0096 & 0.0036 \\
\hline 3 & 4,6 & 0.0108 & 0.0035 & 0.0012 & 1.0324 & 0.0105 & 0.0036 \\
\hline 4 & 5,4 & 0.0088 & 0.0030 & 0.0012 & 1.0264 & 0.0090 & 0.0036 \\
\hline 5 & 5,5 & 0.0127 & 0.0040 & 0.0013 & 1.0381 & 0.0120 & 0.0039 \\
\hline 6 & 5,6 & 0.0097 & 0.0043 & 0.0013 & 1.0291 & 0.0129 & 0.0039 \\
\hline 7 & 6,4 & 0.0126 & 0.0032 & 0.0013 & 1.0378 & 0.0096 & 0.0039 \\
\hline 8 & 6,5 & 0.0112 & 0.0035 & 0.0013 & 1.0336 & 0.0105 & 0.0039 \\
\hline 9 & 6,6 & 0.0100 & 0.0030 & 0.0013 & 1.0300 & 0.0090 & 0.0039 \\
\hline 10 & 7,5 & 0.0102 & 0.0032 & 0.0013 & 1.0306 & 0.0096 & 0.0039 \\
\hline \multicolumn{2}{|c|}{ Average } & 0.0109 & 0.0034 & 0.0013 & 1.0326 & 0.0102 & 0.0038 \\
\hline
\end{tabular}

Mean of average BAFo per particle: 1.0326

Standard deviation of average BAFo per particle: 0.0039

\section{Comments}
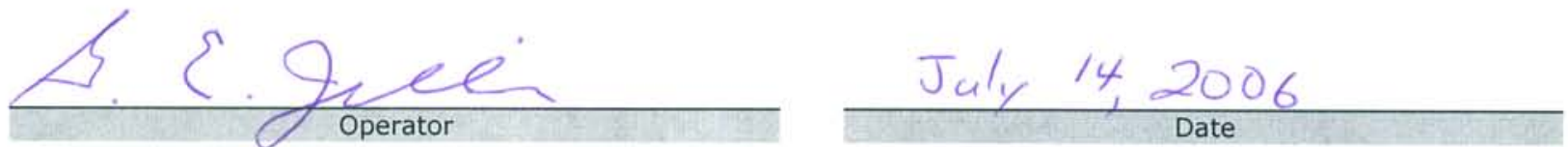
Data Report Form DRF-18B: Measurement of Pyrocarbon Anisotropy using the 2-MGEM - OPyC

\begin{tabular}{|r|l|}
\hline Procedure: & AGR-CHAR-DAM-18 Rev. 1 \\
\hline Operator: & G. E. Jellison \\
\hline Mount ID: & M06062001L \\
\hline Sample ID: & LEU01-46T-Z06 \\
\hline Sample Description: & Particles deconsolidated from AGR-1 baseline compact \\
\hline Folder containing data: & $\backslash \backslash$ mc-agr\AGR\2-MGEM\R06071401\} $\\
{\hline}$
\end{tabular}

\begin{tabular}{|c|c|c|c|c|c|c|c|}
\hline \multirow{2}{*}{ Particle \# } & \multirow{2}{*}{$\begin{array}{c}\text { Grid } \\
\text { Position }\end{array}$} & \multicolumn{3}{|c|}{ Diattenuation } & \multicolumn{3}{c|}{ Equivalent BAFo } \\
\cline { 3 - 8 } & Average & St. Dev. & Ave. Error & Average & St. Dev. & Ave. Error \\
\hline \hline 1 & 4,4 & 0.0117 & 0.0028 & 0.0012 & 1.0351 & 0.0084 & 0.0036 \\
\hline 2 & 4,5 & 0.0104 & 0.0033 & 0.0013 & 1.0312 & 0.0099 & 0.0039 \\
\hline 3 & 4,6 & 0.0123 & 0.0038 & 0.0015 & 1.0369 & 0.0114 & 0.0045 \\
\hline 4 & 5,4 & 0.0116 & 0.0031 & 0.0012 & 1.0348 & 0.0093 & 0.0036 \\
\hline 5 & 5,5 & 0.0106 & 0.0033 & 0.0013 & 1.0318 & 0.0099 & 0.0039 \\
\hline 6 & 5,6 & 0.0121 & 0.0042 & 0.0015 & 1.0363 & 0.0126 & 0.0045 \\
\hline 7 & 6,4 & 0.0115 & 0.0037 & 0.0012 & 1.0345 & 0.0111 & 0.0036 \\
\hline 8 & 6,5 & 0.0111 & 0.0034 & 0.0015 & 1.0333 & 0.0102 & 0.0045 \\
\hline 9 & 6,6 & 0.0092 & 0.0032 & 0.0014 & 1.0276 & 0.0096 & 0.0042 \\
\hline 10 & 7,5 & 0.0109 & 0.0034 & 0.0015 & 1.0327 & 0.0102 & 0.0045 \\
\hline \multicolumn{2}{|r|}{ Average } & 0.0111 & 0.0034 & 0.0014 & 1.0334 & 0.0103 & 0.0041 \\
\hline
\end{tabular}

Mean of average BAFo per particle: 1.0334

Standard deviation of average BAFo per particle: 0.0028

\section{Comments}
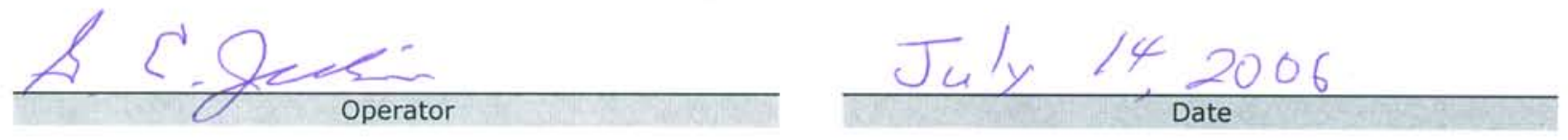


\section{Defective IPyC analysis of LEU01-46T-Z compact lot}

Particles recovered after leach-burn-leach of four sets of 3 compacts each were sandwiched in a single layer between $75 \mu \mathrm{m}$ thick Kapton and imaged with x-rays. Although no particles were identified that exhibited unacceptable uranium dispersion as specified by the visual standard referenced in the AGR-1 fuel specification EDF-4380, many anomalies were noted during the examination of the $\mathrm{x}$-ray images.

\section{General comments}

The fuel kernel blocks essentially all the x-rays producing an unexposed (white) circle in the $\mathrm{x}$ ray image. The $\mathrm{SiC}$ partially stops the x-rays. This produces a mostly unexposed (white) ring around the outer edge of the particle where the x-ray path length is the greatest. The thickness of this ring is approximately the thickness of the $\mathrm{SiC}$ layer. Between the outer $\mathrm{SiC}$ ring and the inner kernel circle, the x-ray image typically shows a gradient in the contrast, going from gray at the outer $\mathrm{SiC}$ ring to black close to the kernel circle. The x-ray attenuation in the buffer and inner pyrocarbon (IPyC) layers is negligible.

Distinguishing $\mathrm{x}$-ray attenuation due to uranium dispersion in the buffer and IPyC layer from $\mathrm{x}$ ray attenuation due to the outer $\mathrm{SiC}$ layer is problematic. Initial calibrations to quantify the $\mathrm{x}$-ray images and models for the current analysis system indicate that a dispersion of $1-2 \mathrm{wt} \%$ of the uranium in the kernel may be necessary to produce an image similar to those indicated as showing unacceptable dispersion in the visual standard. However, variations in SiC thickness and deviations from a perfect spherical shape can further complicate this analysis. Figure C-1 shows a series of images generated using an $\mathrm{x}$-ray absorption model assuming a spherical baseline particle (minus OPyC) with various amounts of uranium uniformly distributed at the IPyC/buffer interface.

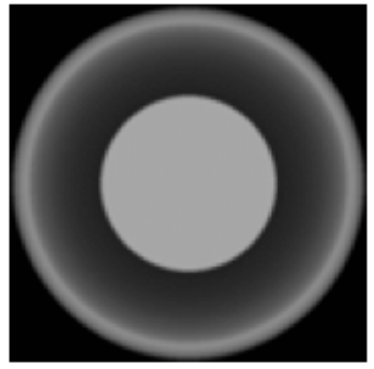

$0.1 \mu \mathrm{g}$

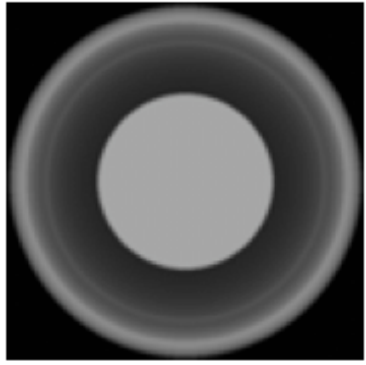

$1 \mu \mathrm{g}$

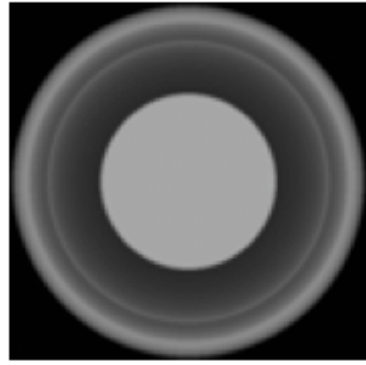

$2 \mu \mathrm{g}$

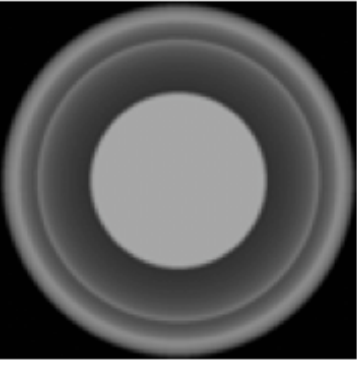

$4 \mu \mathrm{g}$

Figure C-1: Model of x-ray image for AGR-1 baseline particle with various amounts of $U$ migrated to the IPyC/buffer interface. 
Facets in the SiC layer

Facets and dimples in the $\mathrm{SiC}$ layer sometimes result in areas on the image of less exposure inside the $\mathrm{SiC}$ ring, such as can be seen in Figure $\mathrm{C}-2$. This is due to an increased path length in the $\mathrm{SiC}$. These bright streaks are clearly associated with a faceted area in the $\mathrm{SiC}$ layer and are not counted as defective particles for the defective IPyC analysis.

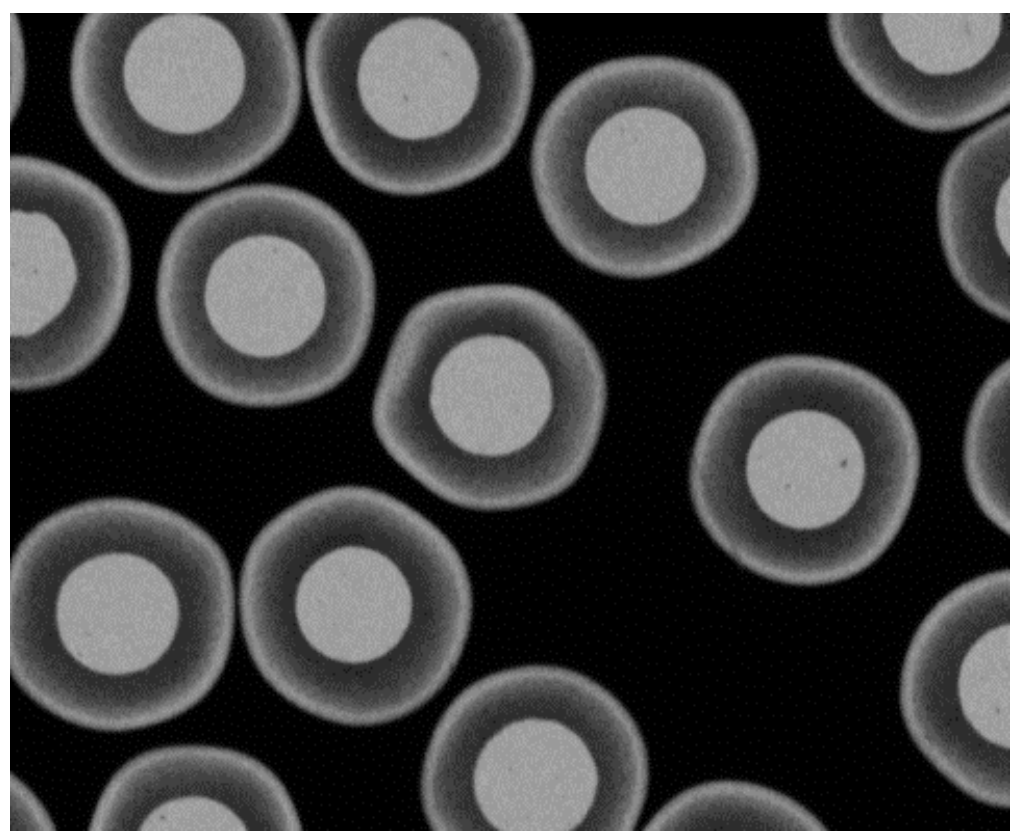

Figure C-2: Faceted or dimpled SiC layer.

Abnormal kernels

One commonly observed anomaly was undersized kernels, ranging between 200 and $300 \mu \mathrm{m}$ diameter. 25 particles were noted to contain noticeably undersized kernels. 25 out of 49735 corresponds to a $95 \%$ confidence anomaly fraction of $\leq 7.1 \cdot 10^{-4}$ for the particles in the compact lot. An example of a particle with a $200 \mu \mathrm{m}$ diameter undersized kernel is shown in Figure C-3. The source of the undersized kernels may be due to the inefficiency of sieving for eliminating small kernels or a kernel forming anomaly where small kernel spheres "hatch out" of normal sized kernels after insertion into the coating furnace. Because smaller particles tend to coat faster, these small kernels end up producing particles that are equivalent in size to the average TRISO and therefore are not removed by the roller-micrometer used to remove over- and undersized particles. 


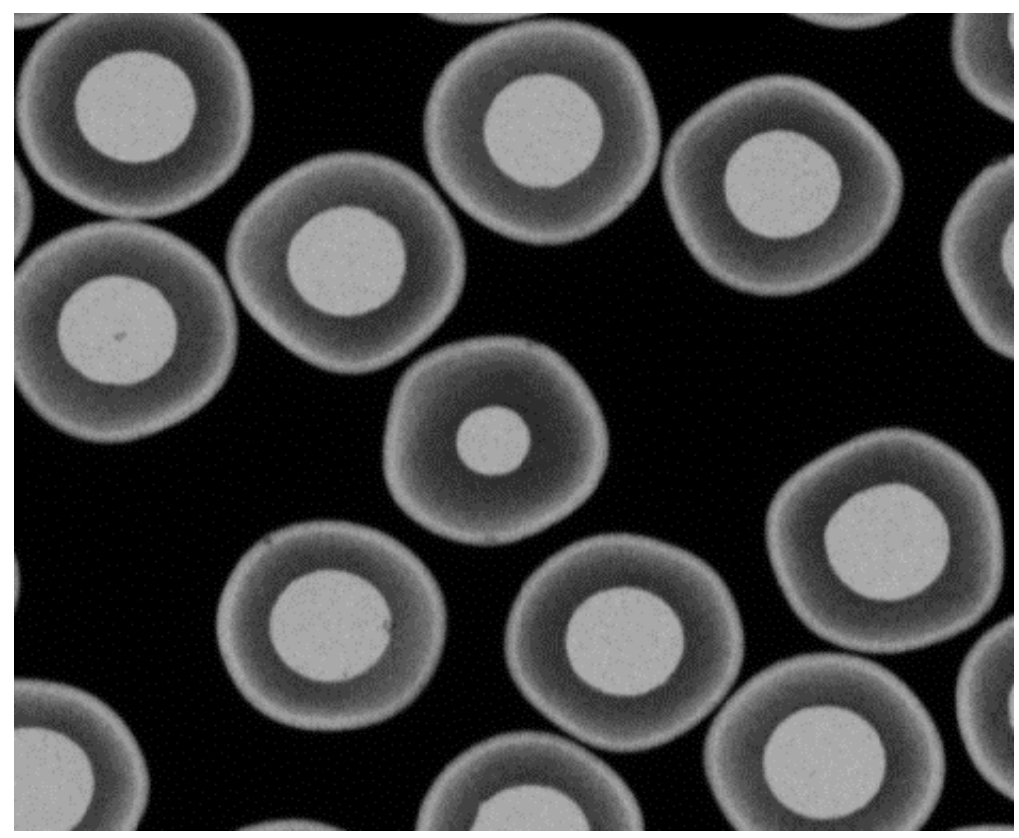

Figure C-3: x-ray image of particle with undersized kernel.

Particles with non-spherical kernels can also be seen in the x-ray images. Small dents and protrusions do not appear to propagate beyond the buffer to affect the shape of the $\mathrm{SiC}$ layer. However, more abnormally shaped kernels do affect the particle shape as in Figure C-4.

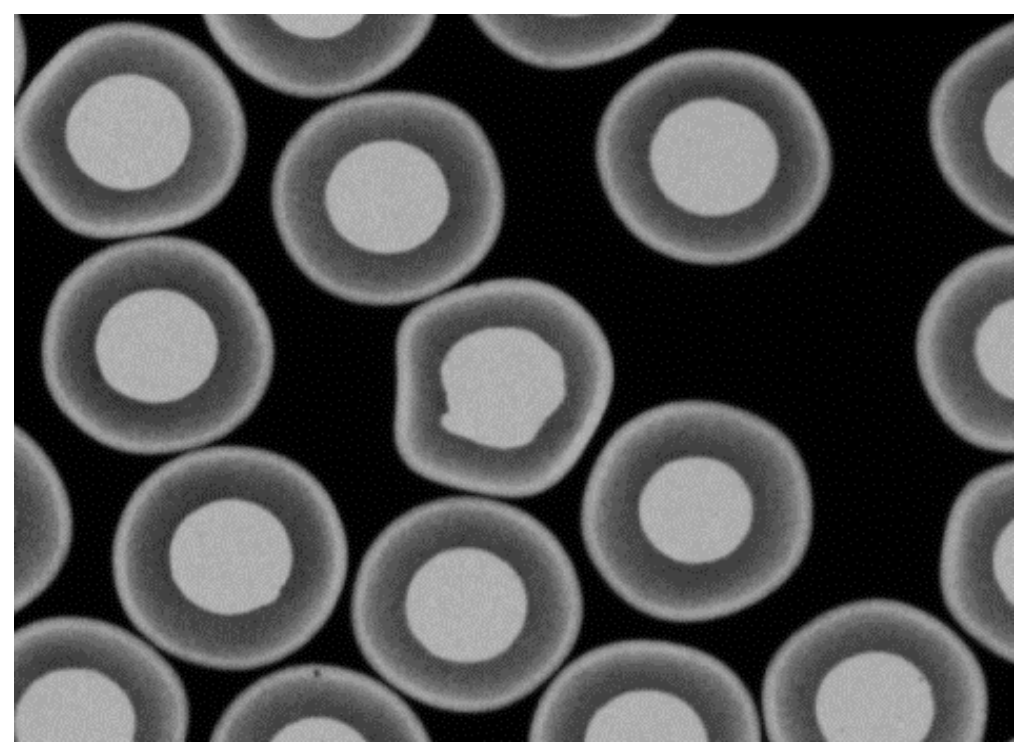

Figure C-4: x-ray image of particle with odd shaped kernel. 


\section{Abnormal SiC}

Another commonly observed anomaly in the x-ray images of the particles was $\mathrm{SiC}$ that was less than half the average thickness of $35 \mu \mathrm{m} .15$ particles were noted to have thin SiC. 15 out of 49735 corresponds to a $95 \%$ confidence anomaly fraction of $\leq 4.7 \cdot 10^{-4}$ for the particles in the compact lot. An example of a particle with approximately $15 \mu \mathrm{m}$ thick $\mathrm{SiC}$ is shown in Figure $\mathrm{C}$-5. In some of the particles with thin $\mathrm{SiC}$, the $\mathrm{SiC}$ thickness clearly varied around the particle (Figure $\mathrm{C}-6$ ). The thin $\mathrm{SiC}$ is probably caused by particles being ejected from the coating zone of the fluidized bed and temporarily adhering to the walls of the coating chamber.

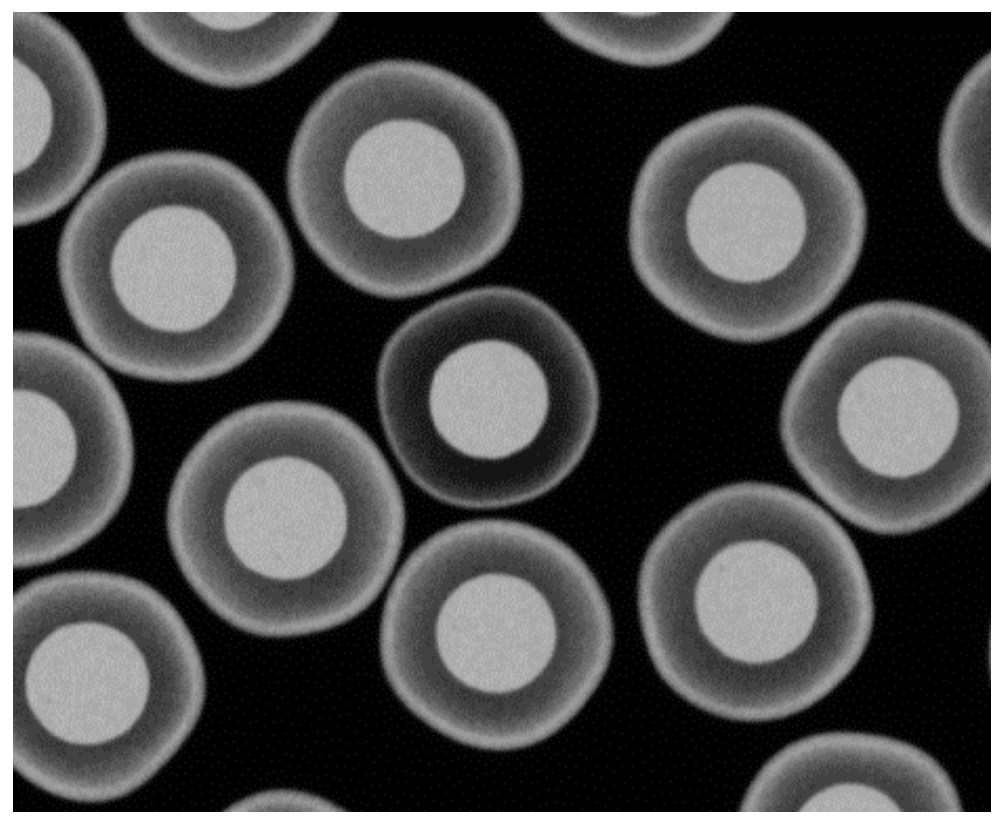

Figure C-5: x-ray image of particle with thin SiC.

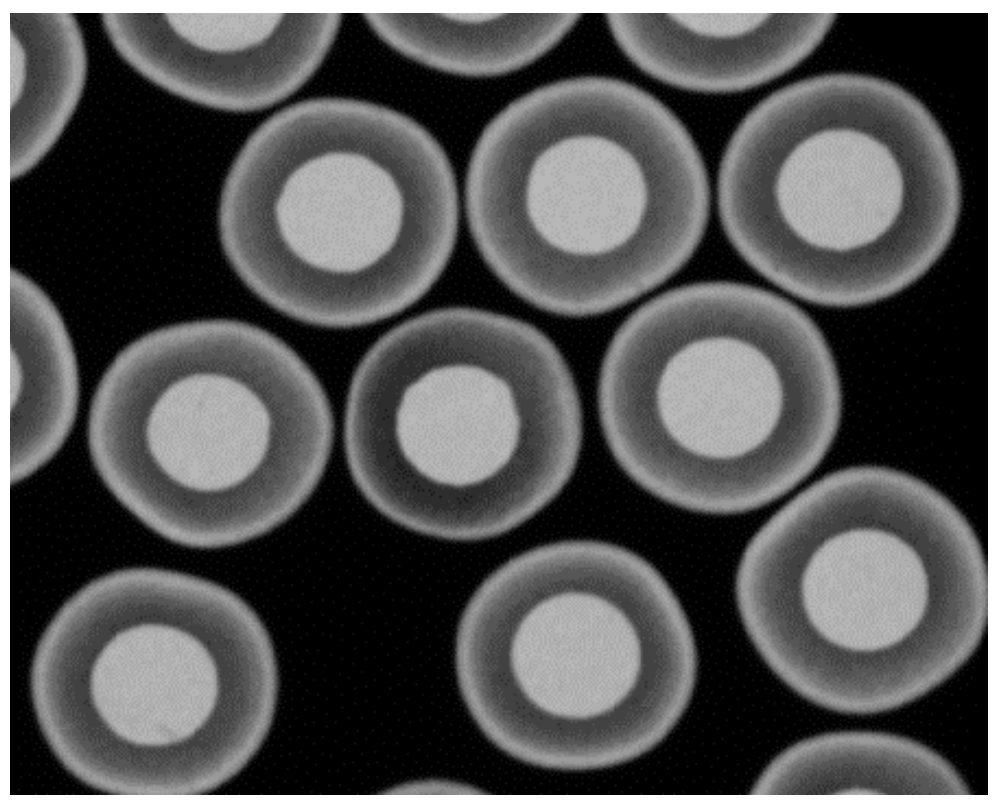

Figure C-6: x-ray image of particle with uneven SiC thickness. 


\section{Abnormal particle shapes}

Four particles were noted that exhibited large protrusions, such as shown in Figure C-7. This anomaly is probably caused by the inclusion of carbon soot at the $\mathrm{IPyC} / \mathrm{SiC}$ interface. These types of particle would normally be removed by the shape separation table, but it is known that the tabling is not $100 \%$ effective.

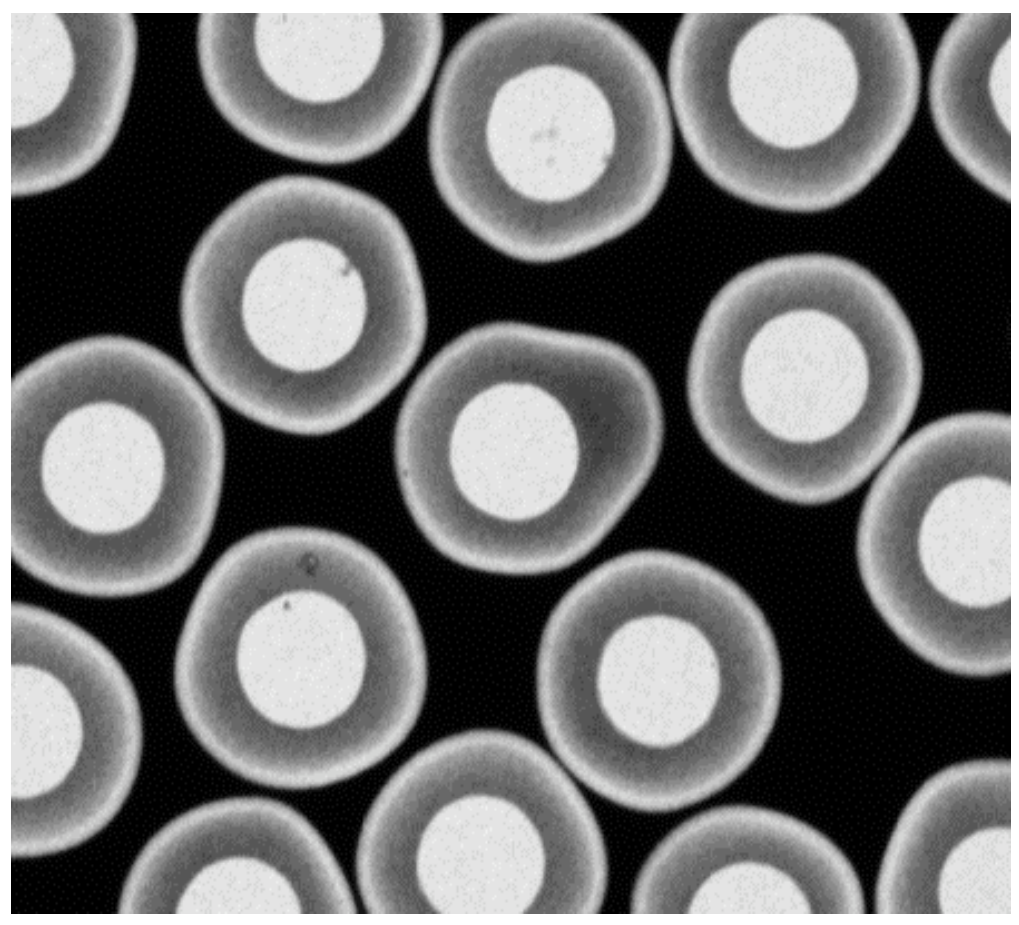

Figure C-7: x-ray image of a particle with a large protrusion. 


\section{Leach-Burn-Leach defects}

Two of the four sets of 3 compacts exhibited 1 leach-burn-leach (LBL) defect each. The LBL defective particles were evident in the x-ray imaging of the particles recovered from that analysis. Figure C-8 shows a particle from compacts LEU01-46T-Z $(40,48,64)$ where the kernel was removed by the nitric acid leach through a defect in the $\mathrm{SiC}$ which is not apparent in the $\mathrm{x}$ ray image. Figure $\mathrm{C}-9$ shows some $\mathrm{SiC}$ fragments from a particle in compacts LEU01-46T$\mathrm{Z}(10,13,66)$.

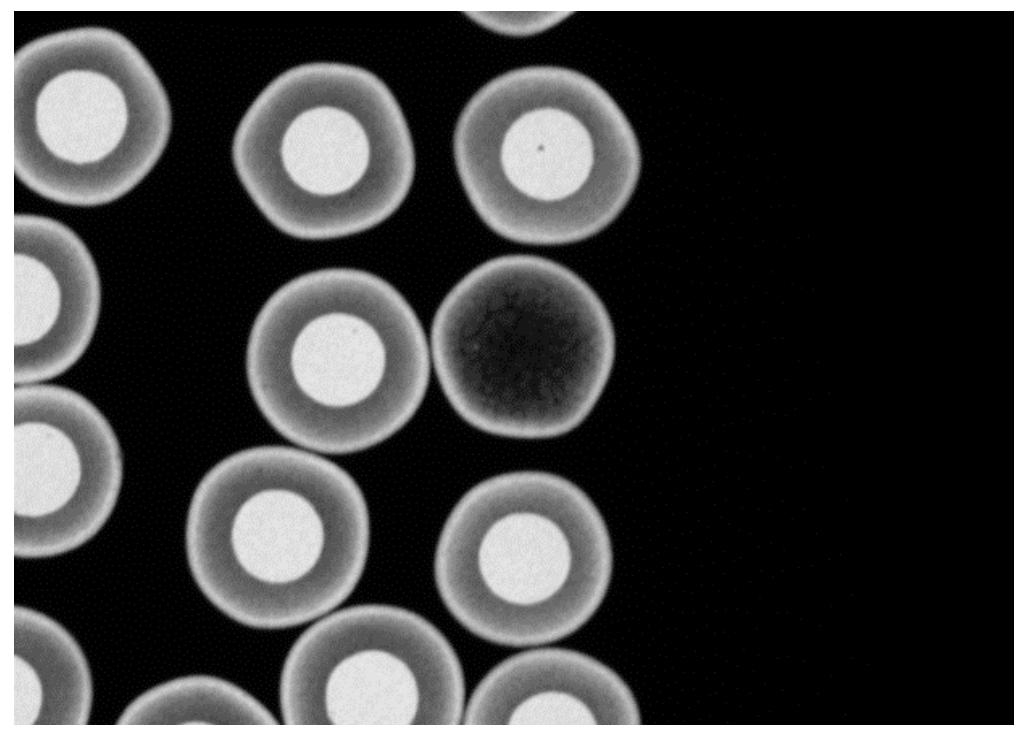

Figure C-8: x-ray image of LBL defect particle with leached out kernel.

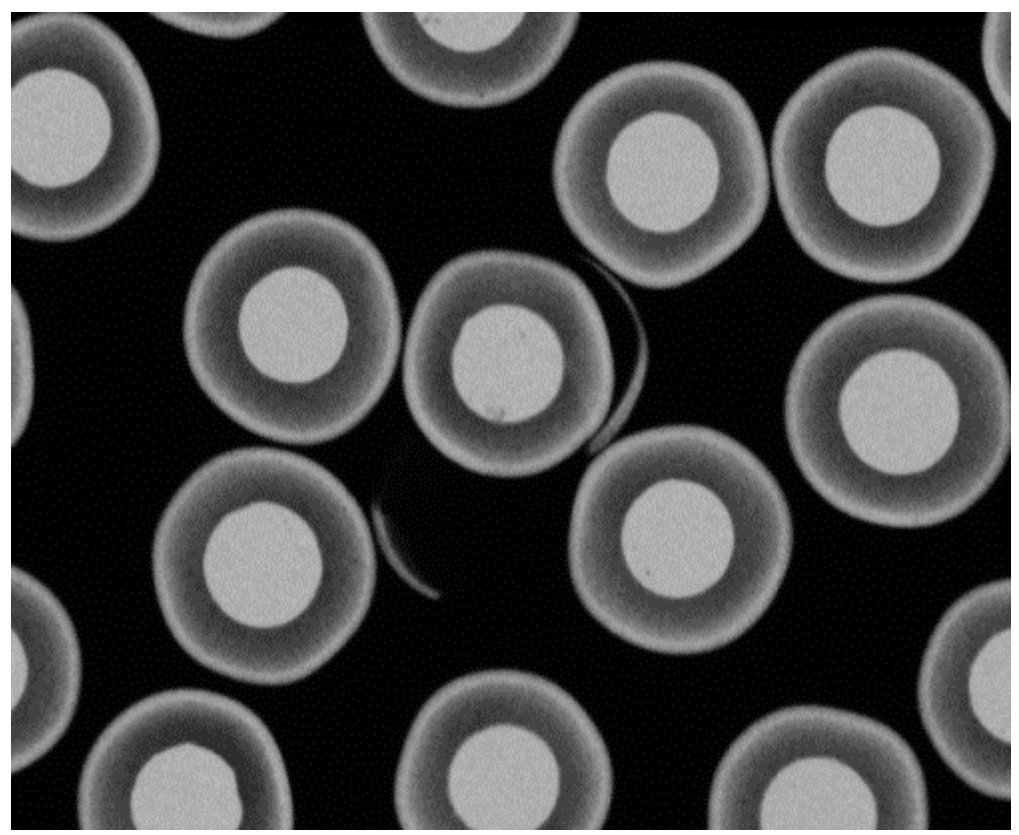

Figure C-9: x-ray image of SiC fragments from LBL defect particle. 